\title{
Secondary prevention in coronary artery disease : evaluation of a behavioral intervention aimed at the reduction of risk factors in coronary patients
}

Citation for published version (APA):

Sebregts, E. H. W. J. (2004). Secondary prevention in coronary artery disease : evaluation of a behavioral intervention aimed at the reduction of risk factors in coronary patients. [Doctoral Thesis, Maastricht University]. Universiteit Maastricht. https://doi.org/10.26481/dis.20041022es

Document status and date:

Published: 01/01/2004

DOI:

10.26481/dis.20041022es

Document Version:

Publisher's PDF, also known as Version of record

Please check the document version of this publication:

- A submitted manuscript is the version of the article upon submission and before peer-review. There can be important differences between the submitted version and the official published version of record.

People interested in the research are advised to contact the author for the final version of the publication, or visit the DOI to the publisher's website.

- The final author version and the galley proof are versions of the publication after peer review.

- The final published version features the final layout of the paper including the volume, issue and page numbers.

Link to publication

\footnotetext{
General rights rights.

- You may freely distribute the URL identifying the publication in the public portal. please follow below link for the End User Agreement:

www.umlib.nl/taverne-license

Take down policy

If you believe that this document breaches copyright please contact us at:

repository@maastrichtuniversity.nl

providing details and we will investigate your claim.
}

Copyright and moral rights for the publications made accessible in the public portal are retained by the authors and/or other copyright owners and it is a condition of accessing publications that users recognise and abide by the legal requirements associated with these

- Users may download and print one copy of any publication from the public portal for the purpose of private study or research.

- You may not further distribute the material or use it for any profit-making activity or commercial gain

If the publication is distributed under the terms of Article $25 \mathrm{fa}$ of the Dutch Copyright Act, indicated by the "Taverne" license above, 


\section{SECONDARY PREVENTION IN CORONARY ARTERY DISEASE}

EVALUATION OF A BEHAVORIAL. INTERVENTION AIMED AT

THE REDUCTION OF RISK FACTORS IN CORONARY PATIENTS 
ISBN: $90-9018196-2$

Lay Our: Jeanne Toelanie, 's-Hertogenbosch

Cover: Maria Sibylla Merian (1647-1717)/Metamorphosis insectorum Surinamensium

Cover Design: Jacky Rinket, Maastricht

Printing: Datawyse BV, Maastriche

(C) EHWJ Sebregts, 2004

Ali rights reserved. No part of this thesis may be reproduced or transmitted in any form or by any means, electronic or mechanical, including photocopying, recording or any information, storage or retrieval system, without permission of writing from the author, or, when appropriate, from the publishers of the publications. 


\section{SECONDARY PREVENTION IN CORONARY ARTERY DISEASE}

EVALUATION OF A BEHAVIORAL INTERVENTION AIMED AT THE REDUCTION OF RISK FACTORS IN CORONARY PATIENTS

\section{PROEFSCHRIFT}

ter verkrijging van de graad van doctor aan de Universiteit Maastricht, op gezag van de Rector Magnificus, Prof. Mr. G.P.M.F. Mols

volgens het besluit van het College van Decanen, in het openbaar te verdedigen

op vrijdag 22 oktober 2004 om 14.00 uur.

door

Ellen Helena Wilhelmina Johanna Sebregts 


\section{PROMOTORES}

Prof. dr. A. Appels

Prof. dr. F.W.H.M. Bär

\section{BEOORDELINGSCOMMISSIE}

Prof. dr. H.W. van den Borne (voorzitter)

Prof. dr. J. Denollet (Universiteit van Tilburg)

Dr. R. van Diest

Prof. dr. T, van Elderen (Universiteit Leiden)

Dr. I.M. Hellemans (Alant Cardio, Amsterdam)

The study presented in this thesis was conducted at the Department of Medical, Clinical and Experimental Psychopathology and was embedded in the Cardiovascular Research. Institute Maastricht (CARIM), Maastricht University, The Netherlands. CARIM was acknowledged in 1992 by the Royal Netherlands Academy of Arts and Sciences (KNAW). The study described in this thesis was supported by a grant of the Netherlands Heart Foundation (94.095)

Financial support by the Netherlands Heart Foundation for the publication of this thesis is gratefully acknowledged.

Publication of this thesis was also made possible by financial support from Stichting Hartsvrienden Rescar. 


\section{CONTENTS}

INTRODUCTION

\section{CHAPTER 1}

Risk factor modification through non-pharmacological interventions

in patients with coronary heart disease - A review.

\section{CHAPTER 2}

Psychological effects of a short behavior modification program in patients with acute myocardial infarction or coronary artery bypass grafting.

\section{CHAPTER 3}

Cholesterol changes in coronary patients after a short behavior modification program.

\section{CHAPTER 4}

Do meta-analyses of the effects of psychoeducational interventions

for coronary patients still give a valid estimate of the effect?

\section{CHAPTER 5}

Is it useful to select coronary artery disease patients for a multifactorial behavioral group intervention?

\section{CHAPTER 6}

General discussion.

REFERENCES

SUMMARY 



\section{INTRODUCTION}

Coronary artery disease (CAD) remains the principal cause of death despite reductions in CAD mortality that have been observed over the past decades in developed countries (McGovern et al., 2001; Rosamond et al., 1998). Declining rates of first and recurrent acute myocardial infarction. (AMI) seem one of the factors contributing to the decreasing trend in CAD mortality (Tunstall-Pedoe et al., 1999), which is probably the result of both primary and secondary prevention strategies such as improved medical treatment. Average overall in-hospital mortality after AMI decreased from $29 \%$ during the 1960 s to $21 \%$ during the 1970 s and to $16 \%$ during the 1980 s (Widdershoven \& De Vreede-Swagemakers, 1997). In-hospital mortality further decreased to $10 \%$ in 1988 , and remained the same in 1994 despite further aging of the population. The impact of medication on the decline in CAD mortality has been described as being substantial (Hunink et al., 1997).

The positive impact of medication regimens on preventing or postponing recurrent coronary events should not in any way be trivialized. Drugs, however, may exert annoying side effects, which may decrease patients' quality of life, or even restrain patients from complying with prescribed medication regimens. A second approach to the reduction of CAD risk factors is by means of interventions aimed at behavior modification. After all, many of the risk factors for CAD are either directly or indirectly related to lifestyle. For example, smoking, elevated serum cholesterol levels, high blood pressure, obesity and lack of physical exercise are established CAD risk factors. Psychological factors, such as hostility, vital exhaustion and depression have been found to exert elevated risk as well (FrasureSmith, Lespérance, \& Talajic, 1995; Kop, Appels, Mendes de Ieon, de Swart, \& Bär, 1994; Miller, Smith, Turner, Guijarro, \& Hallet, 1996). Moreover, multuple risk factors often act in a complex and synergetic way.

In the past, several trials have been conducted to bring about behavior change in coronary patients in order to reduce subsequent risk of a recurrent event. A metaanalysis suggested that health education and stress management programs for coronary patients yielded a significant reduction in mortality and recurrence of myocardial infarction (Dusseldorp, Van Elderen, Maes, Meulman, \& Kraaij, 1999). Moreover, these positive effects were moderated by significant favorable changes in risk factors.

During the past decades, several studies were conducted that intended to improve risk factors in CAD patients. Some of these studies, which evaluated long-term comprehensive interventions, reported promising results concerning reduced risk factors and even cardiac mortality. Although the study is restricted by methodological shortcomings since the patient's informed consent was asked after randomization had been performed - leading to a clear selection bias - the Lifestyle Heart Trial reported substantial positive effects on cholesterol levels after a longterm behavioral intervention, with subsequent positive changes in atherosclerotic lesions (Ornish et al., 1990). In the Recurrent Coronary Prevention Project 
(RCPP), a long-term intervention brought about significant reduction of type $A$ behavior after 1 year and 4,5 years of follow-up, and this was found to be related to fewer cardiac recurrences (Friedman et al., 1986). In the RCPP, CAD patients in the intervention group received 62 type A group-counseling sessions of 90 minutes duration during 4,5 years, of which 20 sessions were offered during the first year. A study by Burell et. al (Project New Life) supported the idea that reduction of type A behavior can be achieved in AMI patients, after 35 90-minute sessions spread over I year (Burell et al., 1994). Reduction in type A behavior was associated with reduced cardiac morbidity and mortality in CABG patients, after 17 three-hr group sessions held over 1 year followed by 5-6 booster sessions during each of years 2-3 (Burell, 1996).

These results induced us to develop a more concise ( 8 weekly 2,5-hr sessions followed by 3 booster sessions) intervention that was based on the most salient and promising trials of the past decades, i.e. the RCPP, the Lifestyle Heart Trial, and Project New Life. The RCPP had been conducted in order to study the feasibility of altering type $A$ behavior after AMI. The treatment program in the RCPP was based on an expanded cognitive social learning model, and conceptualization of type $\mathrm{A}$ behavior and hostility considered cognitive factors, behavioral factors, physiologic factors and environmental factors. Treatment in the cognitive area consisted of self-instructional training, evaluation of basic cognitions, and mental relaxation. Behavioral treatment included behavioral drills to alter physical activities characteristic of type $A$ behavior. In the environmental area, features of the patients' social environment were evaluated, whereas in the physiologic aspect of the treatment program patients were informed about biochemical processes in stress reactions. Project New Life was a behavioral secondary prevention program aimed at modifying type $\mathrm{A}$ behavior in CABG patients. The interventions used in Project New Life were patterned after the RCPP. The Lifestyle Heart Trial evaluated a comprehensive lifestyle program for patients with CAD, which included a low-fat vegetarian diet $(10 \%$ of calories as fat), moderate aerobic exercise, stress management training, smoking cessation and group support.

The intervention we developed was primarily based on the methods of these three major long-term clinical trials. Contrary to these apparently successful trials, however, we intended to develop an intervention that would be remarkably shorter and thereby presumably cheaper and easier to implement in general health care. The intervention was developed during the years 1992-1995 at the Academic Hospital of Maastricht, and was aimed at risk factor modification and behavioral change in coronary patients.

Guidelines for cardiac rehabilitation increasingly stress the importance of individually tailored treatments (British Association for Cardiac Rehabilitation, 1995; Nederlandse Vereniging voor Cardiologie en de Nederlandse Hartstichting, 1995). Studies that prove the effectiveness of tailored programming are, however, lacking. For this reason, we distinguished patients that were likely to benefit from the intervention from patients that were not likely to benefit from it, planned on 
the basis of risk assessment and individual need. It was assumed that patients with multiple risk factors would profit most from the intervention.

The present study was carried out to answer two questions: (1) Does this cardiac rehabilitation and secondary prevention program contribute to a favorable reduction in clinical risk factors and psychological distress? (2) Is it useful to select $\mathrm{CAD}$ patients for this intervention? We presumed that the intervention would be effective in reducing modifiable risk factors for CAD and emotional distress. Moreover, we expected that patients, who were selected as patients with relatively unfavorable risk factor profiles, would profit more from the intervention than patients who were not selected as such.

Chapter 1 of this thesis starts with an overview of nonpharmacological interventions, which have aimed to reduce CAD risk factors. In chapters 2 and 3 , the effects of the intervention concerning psychological and clinical risk factors are studied. The subject of chapter 4 differs from the other chapters in its subject. We originally intended to report on the effects of the intervention on morbidity and mortality rates, but since hardly any patients died or had recurrent myocardial infaretions during follow-up, no firm conclusions could be drawn. We postulated that since mortality rates are decreasing in time because of improved medical treatments, it would be harder for recent studies to report on the effects of interventions on mortality rates than it had been for older studies. This issue eventually led us to study the influence of secular trends on the effects of psychoeducational intervention studies for patients with CAD, which is described in chapter 4 . In chapter 5 , the use of pre-selecting patients for the multifactorial intervention is discussed. The thesis is concluded with a general discussion in which implications and tentative explanations of the study results are discussed, and suggestions for future research are made. 

CHAPTER 1

\section{RISK FACTOR MODIFICATION THROUGH NON-PHARMACOLOGICAL INTERVENTIONS IN PATIENTS WITH CORONARY HEART DISEASE - A REVIEW}

Published as:

Sebregts, E.H.W.J., Falger, P.R.J., Bär, F.W.H.M. (2000). Risk factor modification through nonpharmacological interventions in patients with coronary heart disease. Journal of Psychosomatic Research, 48, 425-441. 


\section{ABSTRACT}

Coronary Heart Disease (CHD) is still the main cause of death in developed countries. Because of improved treatment many patients survive the acute phase of a myocardial infarction, which makes secondary prevention of CHD of major importance. Most risk factors responsible for the development and progression of CHD are associated with behavior. Therefore, interventions aimed at behavior change may contribute to risk factor modification and secondary prevention of CHD. The effects of separate risk factor modification efforts by means of randomized-controlled clinical trials of non-pharmacological interventions in patients suffering from CHD are reviewed. Interventions aimed at healthy lifestyles may stimulate smoking cessation rates, reduce elevated serum total and LDL cholesterol concentrations and favorably modify type A behavior in CHD patients. Moreover, reduction of coronary atherosclerosis has been reported after intensive lifestyle and exercise interventions, whereas exercise and type $\mathrm{A}$ behavior interventions may also lead to reduced CHD morbidity and mortality. As for hypertension and obesity, studies aimed at secondary prevention are lacking. 


\section{INTRODUCTION}

Coronary heart disease (CHD) is still the single main leading cause of death in most modern, industrialized countries, even though data suggest that mortality rates from CHD are declining (Thom, 1989). This decrease in CHD mortality may be explained by primary prevention efforts and by a decline in population risk factor levels, particularly lower smoking rates and beneficial changes in dietary habits (Beaglehole, 1990). Most of the decline, however, is primarily a consequence of reduced mortality among patients with acute myocardial infarction (AMI). This is the result of more intense cardiologic treatment and improvements in the treatment of patients through risk factor modification (Hunink et al., 1997). It thus seems that the declining mortality can largely be explained by better management of patients with established CHD. This also explains why the number of hospitalizations from CHD is steadily growing, in spite of reduced mortality rates. More adults survive their AMIs when middle-aged, and consequently, the patient's life span is lengthened. They may, however, suffer more often from heart failure when older. Moreover, as the general population ages, the rise in hospitalizations will increase accordingly.

In recent years, researchers and clinicians have tried to counteract the rise in CHD by means of primary and secondary prevention strategies, for instance by promoting behavioral change. Secondary prevention of CHD is thus of major importance, since so many patients survive their AMIs. Clinical treatment of (CHD patients consists primarily of pharmacological therapy (i.c., B-blockers, aspirin, ACE inhibitors, lipid-lowering agents, etc.), whereas only limited advice is given concerning behavioral change, such as smoking cessation. This is reason for concern, since most risk factors responsible for the development or acceleration of coronary atherosclerosis are generally associated with behavior and cognitions. Smoking, lack of physical exercise and inadequate coping with 'stress', for instance, are preventable forms of risk behavior, whereas elevated serum cholesterol, obesity and hypertension often correlate with an unhealthy diet. Interventions aimed at behavioral change instead of - or adjunct to - pharmacological therapy may have several advantages, one of which is that harmful side effects of medication could be diminished, and as a consequence, life quality improved (Jacob, Wing, \& Shapiro, 1987; Linden \& Chambers, 1994; Shapiro \& Jacob, 1983).

The purpose of the present paper is to answer the following two-part question: Do non-pharmacological interventions actually contribute favorably a) to risk factor modification in patients with CHD, and b) in doing so, to a reduction of mortality, morbidity or to favorable changes in atherosclerotic stenosis? Meta-analysis would have been difficult to perform, because of the substantial diversity in study methods and interventions, and the relatively small number of studies performed with coronary patients. 


\section{METHODS}

A computer search was performed on MEDLINE and PSYCHlit, and relevant articles mentioned with the references but not found in these files were also used. (Key words included smoking, cholesterol, diet, physical exercise, training, type $A$ behavio(u)r, hypertension, blood pressure, obesity, weight, non-pharmacological, program(me), intervention, CHD, myocardial infarction, CABG.)

We restricted ourselves to non-pharmacological interventions aimed at modification of smoking, serum cholesterol, physical exercise, type A behavior, hypertension, or body weight.

The following criteria were used for inclusion of a trial in the review:

1) Treatment condition in which patients were offered a non-pharmacological intervention aimed at CHD risk factor modification. Non-pharmacological interventions included: a) counseling/advice concerning health behavior; b) cognitive-behavioral approaches to risk factor modification. Interventions based on these approaches attempt to alter behavior by modifying the antecedents and consequents of that behavior, and by involving people's cognitions in behavior changes; c) interventions not incorporating cognitive or behavioral techniques, but aimed at direct risk factor reduction, e.g. offering exercise training or a lipidlowering diet. 2) Treatment focused on changing one risk factor in particular. Interventions focusing on multiple risk factors were only included as far as one of these risk factors was the main object of change. 3) Study population consisting of patients with established CHD, i.e., patients with angiographically documented coronary artery disease, or patients who had had PTCA, AMI, or CABG. 4) One or more control conditions. 5) Random assignment to conditions. 6) Documentation of outcomes had to include at least modification of one of the above-mentioned risk factors, reduction of mortality, morbidity or favorable changes in atherosclerotic stenosis (i.e., regression, stabilization, or prevention of progression).

It was intended to include controlled, randomized, non-pharmacological trials on hypertension and obesity reduction in CHD patients. However, our computer search did not yield any publications in which the effects of blood pressure reduction or weight loss were studied as secondary prevention, by means of nonpharmacological treatment. Consequently, these risk factors were omitted from discussion. Nevertheless, primary prevention studies suggest that blood pressure reduction can be achieved through non-pharmacological interventions. Programs amed at exercise/weight reduction in particular may result in substantial favorable effects on blood pressure, comparable with those achieved with drug treatment (Jacob, Chesney, Williams, Ding, \& Shapiro, 1991). Stress management techniques seem to result in reduced blood pressure levels as well, but effects are generally modest (Jacob et al., 1991; Jacob et al., 1987; Shapiro, Hui, Oakley, Pasic, \& Jamner, 1997; Yen, Patrick, \& Chic, 1996).

For more precise information about the contents of the randomized controlled trials, the reader is referred to the tables; these include type of intervention, 
number of patients, length of follow-up, and main results. As $p$-values are shown in the table, these will not be mentioned in the text.

\section{SMOKING}

Cigarette smoking is the single most important risk factor for the development and progression of CHD. Smoking appears to accelerate the atherogenic process in both a dose- and duration-dependent manner. Among the damaging effects of smoking on the cardiovascular system are acute increases in blood pressure and coronary vascular resistance, reduction in oxygen delivery, impaired dilation of the coronary vessel wall, enhancement of platelet aggregation, increased fibrinogen. production, and depression of HDL cholesterol (Rigotti \& Pasternak, 1996). Although patients with AMI face a potentially life-threatening situation which can be expected to result in considerable emotional distress, about half or two-thirds: of these patients will still be smoking or resume smoking six months to five years after the incident (Havik \& Macland, 1988; Rigotti \& Pasternak, 1996). Smoking cessation may lead to a significant reduction in mortality in coronary patients, as well as to a reduced rate of hospitalization for AMI (Vlietstra, Kronmal, Oberman, Frye, \& Killip, 1986). Beneficial effects of smoking cessation were described as early as 1975 when Wilhelmsson et al. showed that patients who stopped smoking after a first $\mathrm{AMI}$, reduced their chance of suffering a second nonfatal incident by $50 \%$ ( $<<.01)$; the same applied to cardiovascular mortality $(\mathrm{p}<.05)$ (Wilhelmsson, Vedin, Elmfeldt, Tibblin, \& Wilhelmsen, 1975). Reduced mortality after smoking cessation was confirmed in later studies (Aberg et al., 1983; Daly, Mulcahy, Graham, \& Hickey, 1983; Salonen, 1980; Sato et al., 1992; Sparrow, Dawber, \& Colton, 1978). In coronary patients, the risk of having a coronary event declines soon after smoking cessation and is largely dissipated after 2-3 years. The beneficial effects were found to persist for at least 13 years (Daly et al., 1983; Rosenberg, Palmer, \& Shapiro, 1990; Salonen, 1980; Taylor, Houston-Miller, Haskell, \& DeBusk, 1988).

\section{Secondary prevention trials for coronary patients: implementing smoking cessation}

A number of interventions have been developed to reduce modifiable risk factors for CHD, and smoking was the first factor that became a target for change. This is reflected in the often less than perfect research designs used in the $1.960 \mathrm{~s}$ and 1970 s to evaluate these interventions. Therefore, only a few randomized controlled studies were found that studied the effects of non-pharmacological programs on smoking cessation in coronary patients (table 1). Taylor et al. determined the influence of exercise training on smoking behavior in patients who had suffered an AMI (Taylor et al., 1988). At 6 months follow-up, the prevalence of smoking did not differ significantly between regular exercise and no-exercise or controls. 
However, cigarette consumption was significantly less in patients who exercised than in control patients.

Later, Taylor et al. published a different type of intervention for smoking cessation (Taylor, Houston-Miller, Killen, \& DeBusk, 1990). This time, the intervention was offered during hospitalization due to AMI and was maintained thereafter for 5 months, through regular telephone contact. One year after AMI, the smoking cessation rate was significantly higher in the intervention group.

Ockene et al. studied the effectiveness of a similar type of intervention in patients with angiographically documented CHD (Ockene et al., 1992). The program was initiated in the hospital, and was continued after discharge through counseling visits or by telephone calls. Compared to controls, slightly more intervention patients quit smoking. Sub-analysis showed further that the intervention was more effective in patients with more severe CHD, who had been admitted with AMI.

Only a few programs focus exclusively on smoking cessation. Some multimodal programs incorporated multiple risk factors, including smoking, and have found conflicting results. Some of these programs have found significantly higher smoking cessation rates in coronary patients receiving intervention compared to control patients (DeBusk et al., 1994; Van Elderen-Van Kemenade, Maes, \& Van den Broek, 1994).' Other studies, however, did not find such results (Allen, 1996; Carlsson, Lindberg, Westin, \& Israelsson, 1997). Participation of the partner in the program seemed important in stimulating smoking cessation of coronary patients in one particular study (Van Elderen-Van Kemenade et al. 1994).

In summary, the above-mentioned studies show that non-pharmacological interventions may boost smoking cessation rates in AMI patients. Exercise training by itself may not suffice for stimulating smoking cessation, but behaviorally oriented interventions, incorporating cognitive and behavioral smoking cessation techniques, have shown positive results. Because the interventions consist of different components, it is difficult to determine which components contributed to the favorable effects. However, standard behavioral techniques included in one of these interventions (i.e., teaching patients methods of coping with situations likely to cause relapse) have been shown to be effective in facilitating smoking cessation (Killen, Taylor, \& Maccoby, 1984). In addition, the likelihood of quitting seems to be affected by seriousness of disease; the effects were especially strong in AMI patients. Less is known about the success of smoking cessation interventions in patients after coronary artery bypass graft surgery (CABG). One study showed identical cessation rates in control and intervention groups; half of all patients resumed smoking after surgery, a finding which was not affected by an in-hospital smoking cessation program in the intervention group (Rigotti, McKool, \& Shiffman, 1994). Thus, surprisingly, after more than 25 years of research there still is an urgent need for well-designed studies focusing on smoking cessation in coronary patients.

\footnotetext{
I Studies amed at multuple nsk tactors without partacular focus on smoking cessation are not included in table I
} 
Table 1. Randomized controlled studies on non-pharmacological interventions aimed at smoking cessation in coronary parients.

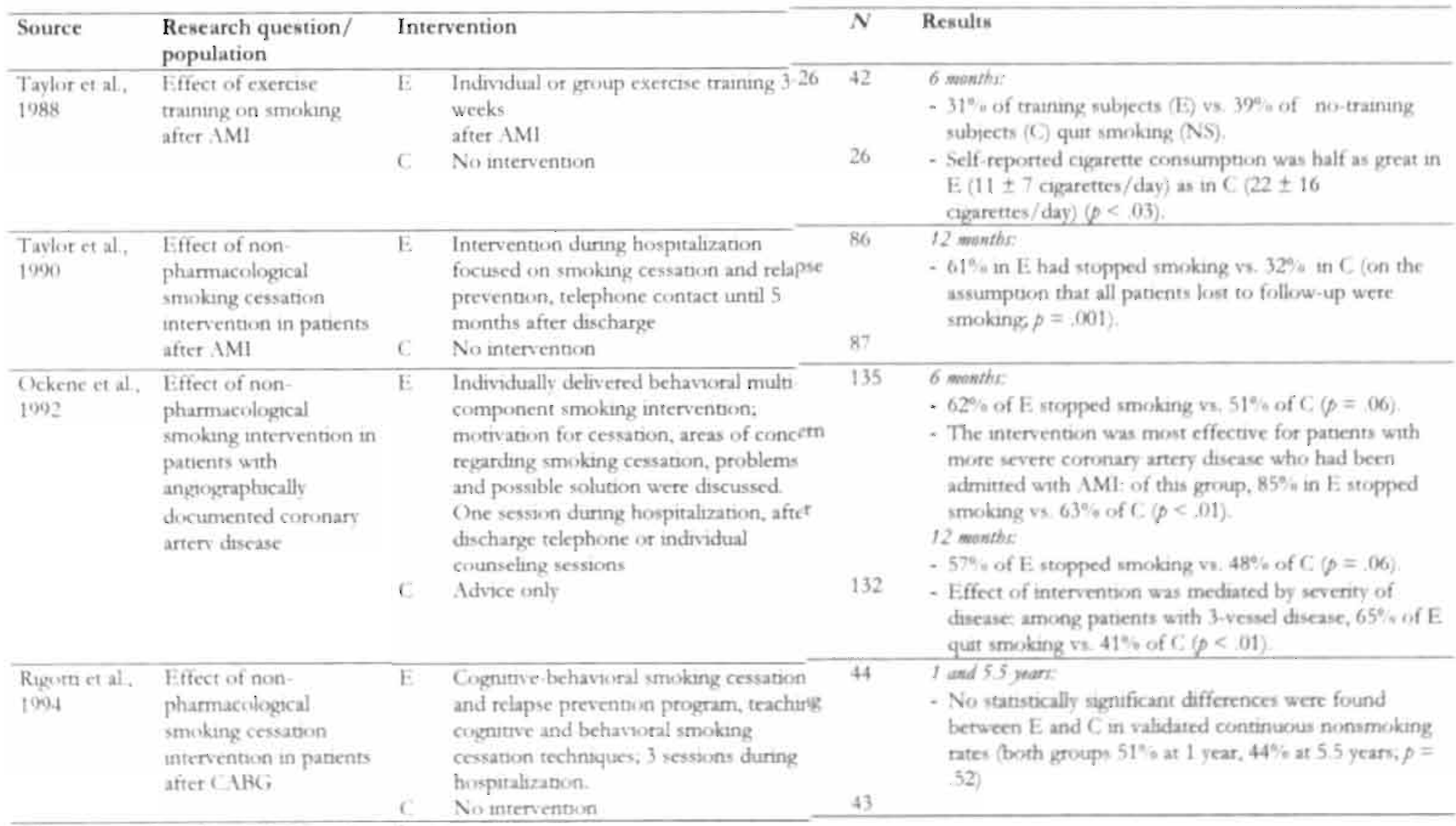




\section{HYPERCHOLESTEROLEMIA}

The essential role of elevated serum cholesterol levels in the etiology of atherosclerosis is now well established (LaRosa et al., 1990; Adult Treatment Panel II, 1994). The increased risk of CHD is determined first by elevated levels of lowdensity lipoprotein (LDL) cholesterol (i.e., $\geq 130 \mathrm{mg} / \mathrm{dL}$ or $\geq 3.4 \mathrm{mmol} / \mathrm{L}$ ), which is thought to promote atherogenesis when oxidized, and second by reduced levels of protective high-density lipoprotein (HDL) cholesterol (i.e., $<35 \mathrm{mg} / \mathrm{dL}$ or $<0.9$ $\mathrm{mmol} / \mathrm{L}$ ), whereas total serum cholesterol level, the HDL/LDL ratio, as well as the level of triglycerides, are of additional importance. Foods that are rich in saturated (animal) fats and cholesterol are the main source of elevated serum cholesterol and increase the risk of CHD, whereas polyunsaturated fatty acids may lower serum cholesterol levels.

Pharmacological treatment of serum cholesterol, especially LDL cholesterol, leads to primary and secondary prevention of cardiovascular incidents and mortality (Gould, Rossouw, Santanello, Heyse, \& Furberg, 1995; Levine, Keaney, \& Vita, 1995). This was most convincingly shown in the Scandinavian Simvastatin Survival Study (Scandinavian Simvastatin Survival Study Group, 1994). Over a 5.4 years follow-up period, simvastatin produced mean reductions in total cholesterol and L.DI. cholesterol of $25 \%$ and $35 \%$, respectively. Moreover, mortality was reduced in coronary patients $(p=.0003)$. Apart from these and other study results, it is becoming increasingly clear that coronary patients with normal and mildly elevated levels of serum cholesterol may benefit as well (Scandinavian Simvastatin Survival Study Group, 1994).

\section{Secondary prevention trials for coronary patients: reducing serum cholesterol}

Already between 1960-1970, several controlled trials studied whether reduction of serum cholesterol by a diet low in saturated fat and cholesterol and rich in polyunsarurated fat would reduce cardiovascular recurrences or improve survival in patients after first AMI or in patients with ischemic heart disease (Leren, 1970; Research Committec, 1965; Research Committee to the Medical Research Council, 1968; Rose, Thomson, \& Williams, 1965; Woodhill, Palmer, Leelarthaepin, MeGilchrist, \& Blacket, 1967). Results were generally disappointing: patients following this diet did not fare any better, and in one study even slightly worse (Rose et al., 1965), than controls. These early studies were, however, characterized by some methodological shortcomings, such as recruitment of low-risk patients only, while drug treatment and baseline cholesterol levels were not reported. Moreover, these trials included very high intakes of polyunsaturated fatty acids, sometimes resulting in a total fat intake of $\geq 40 \%$, despite being low in saturated fats and cholesterol (De Lorgeril, Salen, Monjaud, \& Delaye, 1997). Changes in serum cholesterol levels were in general modest, suggesting that treatment failure might have been caused by inadequate reductions of lipids. The lack of serum 
cholesterol reductions in these early studies reflects the limited knowledge of the cholesterol-atherosclerosis interrelationship in those days.

Recent trials studied the effects of more intensive dietary treatment on cholesterollowering in CHD patients, some of which used objective arteriographic assessment of changes in the size of the coronary arterial lumen before and after intervention (table 2). In 1983, Ornish et al. showed in a randomized pilot study that a strictly vegetarian diet combined with stress-management training resulted in improvements in cardiovascular status, such as clinically meaningful decrease in plasma cholesterol levels and, in particular, a reduction in frequency of anginal episodes (Ornish et al., 1983). The Lifestyle Heart Trial was the first randomized trial to show that these comprehensive lifestyle changes in coronary patients could lead to actual regression of atherosclerosis (Omish et al., 1990). After one year, significant differences were found between intervention and control patients with respect to serum lipid levels and extent of coronary atherosclerosis, in favor of patients in the intervention group. It was concluded that comprehensive lifestyle changes may be able to bring about substantial regression of severe coronary atherosclerosis after only one year, without the use of lipid-lowering drugs. Recent data confirmed ongoing regression in patients who continued to adhere to these lifestyle changes after five years, with a resulting significant reduction in new coronary events by about $250 \%$ compared to controls (Ornish et al., 1998).

Implementation of the Lifestyle Heart Trial intervention program also appeared to be feasible in heart patients in a rehabilitation center in Germany (Scherwitz et al., 1995) and in a primary health care center in the Netherlands (Falger \& Voorhoeve, unpublished). ${ }^{2}$ Results of these latter studies at 3 and 12 months follow-up were rather similar to those of the Lifestyle Heart Trial. However, no conclusions could be drawn regarding regression of coronary artery stenoses, since repeated quantitative coronary arteriography was not performed.

In a different randomized controlled trial, Watts et al. assessed the effects of a lipid-lowering diet, either with or without cholesterol-lowering medication (cholestyramine), on coronary atherosclerosis (Watts et al., 1992). After 3 years, the diet-intervention group showed significant reductions in total cholesterol, LDLcholesterol and triglycerides compared to controls, which did not show any significant changes. In the diet plus cholestyramine group, an additional $10-20 \%$ reduction in cholesterol levels was observed. Compared to controls, the proportion of patients showing overall progression of coronary artery stenoses was significantly reduced by both interventions. These findings support the role of a regular lipid-lowering diet in males with $\mathrm{CHD}$, even when serum cholesterol concentrations are moderately elevated. If necessary, these effects could be enhanced by pharmacotherapy.

\footnotetext{
2Since these studies were not randomized controlled mals, they are not included in table 2.
} 
Table 2. Randomized controlled studies on non-pharmacological intervennons aimed at reduction of serum cholesterol in coronary patients.

\begin{tabular}{|c|c|c|c|c|c|}
\hline Source & $\begin{array}{l}\text { Research question/ } \\
\text { population. }\end{array}$ & \multicolumn{2}{|c|}{ Intervention } & $\mathbf{N}$ & Results \\
\hline $\begin{array}{l}\text { Ornush et al. } \\
1983\end{array}$ & $\begin{array}{l}\text { Effect evaluation of } \\
\text { dietary changes in } \\
\text { combinarion with stress } \\
\text { management training in } \\
\text { patients with ischemic } \\
\text { heart disease }\end{array}$ & C & $\begin{array}{l}\text { Intervention wrth a vegetanan diet } \\
\text { (average daly intake of } 1.400 \text { calones) and } \\
\text { stress management training (i.e. } \\
\text { stretching/relaxation, (applied) } \\
\text { meditarion), during a period of } 24 \text { days } \\
\text { No intervention }\end{array}$ & 23 & 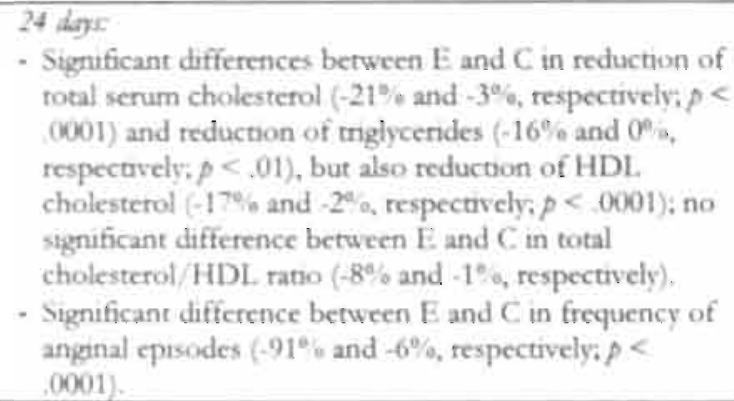 \\
\hline $\begin{array}{l}\text { Ornish er al. } \\
1990\end{array}$ & $\begin{array}{l}\text { To determine whether } \\
\text { comprehenstve lifestyle } \\
\text { changes affect coronary } \\
\text { atherosclerosis in } \\
\text { patients with CHD }\end{array}$ & C & $\begin{array}{l}\text { Intervention amed at lifestyle } \\
\text { modification: low-fat vegetarian diet } \\
\text { ( }<10^{\circ} \text { \% of calones as fat), stress } \\
\text { management training (stretching } \\
\text { /breathing, meditation), moderate } \\
\text { exercise, stopping smoking, group support } \\
\text { No intervention }\end{array}$ & 20 & 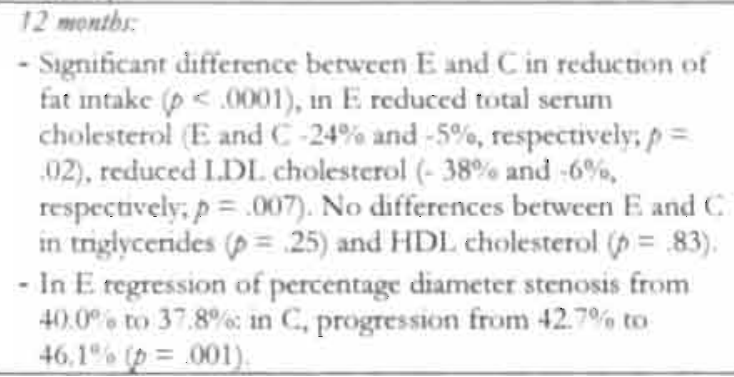 \\
\hline
\end{tabular}

- table continues - 
Table 2 - continucd

\begin{tabular}{|c|c|c|c|c|c|}
\hline Source & $\begin{array}{l}\text { Research question/ } \\
\text { population }\end{array}$ & Int & vention & $N$ & Results \\
\hline $\begin{array}{l}\text { Watts et al. } \\
1992\end{array}$ & $\begin{array}{l}\text { The effect of dictary re- } \\
\text { duction of plasma cho- } \\
\text { lesterol concentrations } \\
\text { on coronary atheroscle- } \\
\text { rosis in panents with } \\
\text { angina or past AMI }\end{array}$ & $\begin{array}{l}\text { E1 } \\
\text { E2 } \\
\text { C. }\end{array}$ & $\begin{array}{l}\text { Lipid-fowering diet: total fat intake was } \\
\text { reduced tri } 27 " 6 \text { of dietary energy } \\
\text { Liped-fowering diet plus cholestyramine } \\
\text { No intervention }\end{array}$ & $\begin{array}{l}26 \\
24 \\
24\end{array}$ & 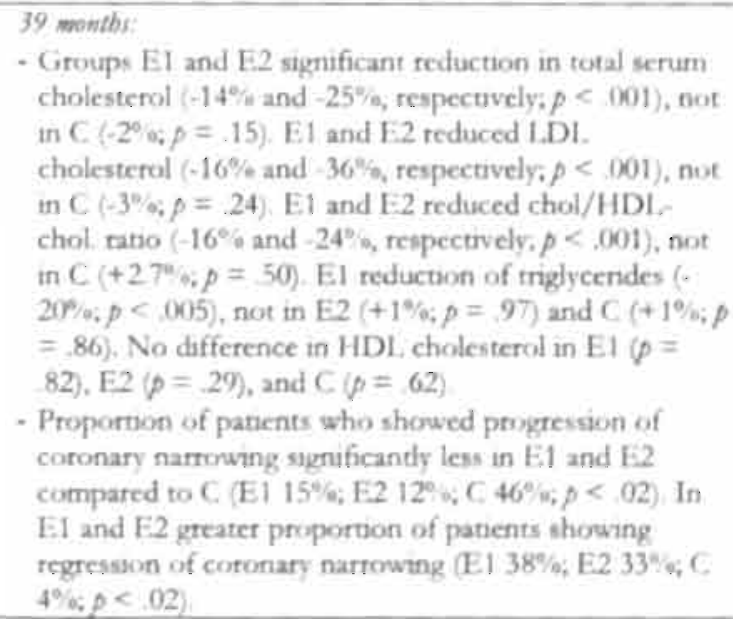 \\
\hline $\begin{array}{l}\text { Singh ef al. } \\
\text { loon: }\end{array}$ & $\begin{array}{l}\text { The effect of a } \\
\text { cardioprotectuve dect on } \\
\text { comphcations and } \\
\text { mortaliry in patients } \\
\text { with suspected AMI of } \\
\text { unstable angina pectons }\end{array}$ & E & $\begin{array}{l}\text { Fat reduced diet (within } 24-48 \text { hours of } \\
\text { AMI) nch tn scilubie dietary fiber, antr. } \\
\text { oxdant vitames and minerals } \\
\text { Fat reduced diet }\end{array}$ & 202 & 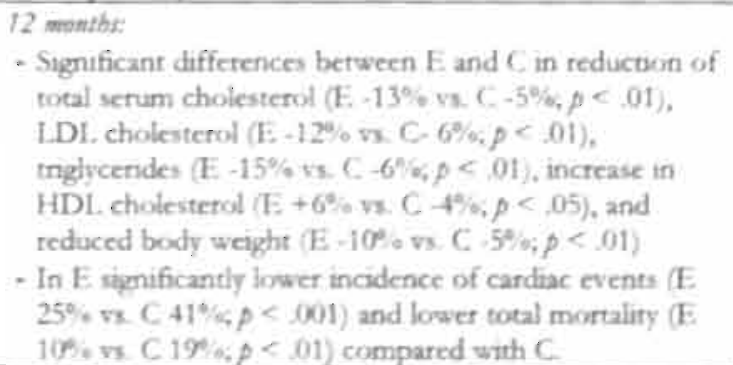 \\
\hline
\end{tabular}

F: Fixpermental condioun: Ci: Control conditom 
Several randomized studies have been conducted that were not primarily aimed at cholesterol reduction, but focused on reduction of multiple risk factors. These trials, in which a low-fat diet was one of several components in the intervention program, reported slower rates of progression of coronary artery stenoses (Haskell et al., 1994; Niebauer et al., 1997). ${ }^{3}$ This was accompanied by significant reductions in LDL cholesterol, plasma triglycerides, and increased HDL cholesterol in only one of the studies, (Haskell et al., 1994) however.

That comprehensive dietary changes in coronary patients may not only lead to reduced serum cholesterol levels and regression of atherosclerotic lesions, but also to reduced cardiovascular complications and mortality, was shown by Singh et al. (Singh et al., 1992). A simple fat reduced diet was compared with a cardiovasoprotective diet reduced in fat and rich in soluble fiber, antioxidants, and minerals. Both diets resulted in weight loss and modest serum cholesterol reductions, but in patients on the cardiovasoprotective diet lipoprotein concentrations showed a larger change and cardiac events also occurred significantly less often. Apart from the Singh et al. trial, two other trials were conducted that were not primarily designed to reduce cholesterol, but questioned whether diets low in saturated fats supplemented with polyunsaturated fats such as fish intake, or supplemented with antioxidant vitamins or fiber, might reduce CHD morbidity and mortality in coronary patients (Burr et al., 1989; De Lorgeril et al., 1994).' These trials were remarkably successful in reducing CHD and overall mortality, whereas the impact of the diets on reducing serum cholesterol was in general modest. Apparently, even without substantial change in the degree of luminal stenoses, modest reductions of serum cholesterol could lead to significant reductions in cardiovascular morbidity or mortality (Scandinavian Simvastatin Survival Study Group, 1994; Singh et al., 1992). These reductions in clinical events could reflect a stabilization of stenotic lesions and inhibition of thrombus formation (Blankenhorn \& Hodis, 1994; Brown, Zhao, Sacco, \& Albers, 1993; Rosenson \& Tangney, 1998).

In sum, these studies showed that elevated serum cholesterol concentrations can be reduced by intensive dietary modifications. No specific behavioral or cognitive techniques were described in any of the studies, to help patients adopt a low-fat diet in everyday life. In two studies interventions consisted of serving a vegan diet to patients during a residential retreat with additional classes in food preparation (Ornish et al., 1990; Ornish et al., 1983), or simply of instructions by a dietician in one study (Watts et al., 1992). It appeared that major changes in dietary habits could be achieved, with positive consequences for the atherosclerotic process. Adopting a strictly vegetarian diet $(<10 \%$ of calories as fat) seems more effective than a less radical dietary change (Ornish et al., 1990). The beneficial effects can be seen after 1 year, may persist much longer, and may already be apparent after three months. It should be noticed, however, that some interventions did not solely consist of a low-fat diet (Ornish et al., 1990; Ornish et al., 1983). The relative contribution of each component of the lifestyle program(s) is, therefore, obscure.

' Is these studies were not primarily atmed at cholesterol reductoon, they are not mentioned in rable 2 
Another point of discussion concerns the impact of a small regression of coronary atherosclerosis on mortality rates among people with CHD. This question remains unanswered, but it seems that even a small amount of regression in a critically stenosed artery has a large effect on myocardial perfusion and thus on functional status (Ornish et al., 1990). In addition, plaque stabilization or other measures might also be a reason for an improved outcome, as has been suggested in the statin trial (Scandinavian Simvastatin Survival Study Group, 1994). All three lipidlowering trials were randomized, controlled clinical trials including an average of 25 patients in each treatment condition (Ornish et al., 1990; Ornish et al., 1983; Watts et al., 1992). As these study populations are rather small, prudence is advised before drawing firm conclusions. Moreover, these relatively small numbers of patients may reflect the drawback associated with trials offering comprehensive lifestyle changes; they attract a selective group of highly motivated patients to participate, but a considerable number of eligible parients may not be reached. This not only causes selection bias, but seriously restricts generalizability of treatment results to the general population of CHD patients as well, and considerations should be made as to which strategy is the most cost-effective one. Despite all this, an intensive lipid-lowering diet does seem advisable for coronary patients, and psychological strategies should be developed to maintain motivation in eligible patients.

\section{LACK OF PHYSICAL ACTIVITY}

Prospective epidemiological studies strongly indicate that an inactive lifestyle is associated with an increased risk of CHD (Berlin \& Colditz, 1990). This association can be explained in part by the relation between lack of exercise and traditional risk factors for CHD. Sufficient daily physical exercise can prevent or reduce obesity. Moreover, regular exercise is associated with reductions in blood' pressure and serum cholesterol levels; the physically active were found to have reduced LDL cholesterol and triglyceride levels, and increased HDI. cholesterol (Blumenthal \& Emery, 1988; NIH Consensus Development Panel on Physical Activity and Cardiovascular Health, 1996; Rigotti, Thomas, \& Leaf, 1983). Independent of these indirect effects through modified traditional risk factors, an active lifestyle does also directly exert favorable physiological effects, such as reduced heart rate during exercise and at rest, and increased muscular oxygenuptake, which eventually leads to reduced myocardial oxygen demand (Bär \& Vonken, 1990). In CHD patients long-term exercise training may even improve ventricular function over time (Giannuzzi et al., 1997; Oberman et al., 1995). Moreover, physical exercise may have favorable psychological effects, such as reduced feelings of depression and anxiety, improved quality of life, and improved mental functioning (Berlin \& Coldizz, 1990; Kugler, Seelbach, \& Krüskemper, 1994). 


\section{Secondary prevention trials for coronary patients: increasing physical exercise}

Despite improved cardiorespiratory fitness and reduction of the traditional CHD risk factors, evidence that exercise diminishes risk of reinfarction or prolongs life in patients with established CHD remains equivocal. Although many studies reported beneficial effects on cardiovascular morbidity and mortality after exercise, differences between exercise and control groups were generally modest and did not always reach statistical significance.

In 1982, May published a review of randomized controlled trials that focused on secondary prevention after AMI through physical training (May, Eberlein, Furberg, Passamani, \& DeMets, 1982). Only studies with sample sizes of more than 100 patients were included. Over a ten-year period, only six studies were identified that met these criteria. Although none of these studies showed statistically significant reductions of mortality between intervention and control groups, all except one showed a positive trend favoring physical training. Grouping of these studies with a total of 2,752 patients showed that increased physical exercise following AMI would lead to a significant $19 \%$ reduction in total mortality, compared with controls. Obviously, although most studies indicate favorable effects of physical training on mortality, individual studies may often be too small to show statistical significance.

To overcome this problem, meta-analyses were conducted. More than 4,000 patients were included in the meta-analyses of Oldridge et al. and $\mathrm{O}^{\prime}$ Connor et al. (O'Connor et al., 1989; Oldridge, Guyatt, Fischer, \& Rimm, 1988). Table 3 shows the studies included in the Oldridge analysis, because these studies consisted of relatively large study samples. Whereas individual study results were not always significant, meta-analysis indicated a significantly reduced mortality ( $\mathrm{O}^{\prime}$ Connor et al. $20 \%$, and Oldridge et al. 24\%) following physical training. No significant differences were found, however, concerning non-fatal recurrent infarctions. Moreover, effects were found only in studies with at least 3 years of follow-up. As not all studies focused exclusively on physical exercise, those effects could possibly have been the result of modification of multiple risk factors (Oldridge et al., 1988). The reduction in mortality may be explained by various mechanisms, e.g., exercise training may improve myocardial perfusion by preventing the progression of coronary artery stenoses to a certain degree in CHD patients (Kubo, Yano, Hirai, Yabuki, \& Machii, 1992; Schuler et al., 1992; Hambrecht et al., 1993). Regression of coronary narrowing, however, may occur only in patients who spend an average of 5 or $6 \mathrm{~h} /$ week of regular physical exercise. These beneficial effects appear to be maintained after 6 years of risk intervention (Niebauer et al., 1997).

Many studies evaluated interventions in which the effects of physical exercise were studied among other risk factors, but only some of these focused primarily on physical exercise. Individual studies focusing on effects of physical reconditioning often encounter several methodological problems: relatively many patients randomized to the experimental groups drop out (resulting in small sample sizes),

\footnotetext{
4 The papers by Schuler et al. (1992), Hambrecht et al. (1993) and Niebauer et al. (1997) were part of one large study: only the Hambrecht study is reported in table 3
} 
or patients in control groups start training by themselves. To increase statistical power, meta-analyses have been conducted, which have shown statistically significant reductions of mortality after physical exercise. Meta-analyses can, however, lead to false conclusions, because of publication bias (studies showing negative results are less likely to be published). Moreover, often study designs and patient samples of individual studies lack comparability, which could lead to false positive conclusions.

Thus, unequivocal evidence for the favorable effects of physical exercise on cardiovascular morbidity is not yet available, although cardiovascular mortality seems to be reduced. Unfortunately, those interventions (generally) offered supervised group exercise programs. to patients, but were not aimed at stimulating individual exercise in everyday life, e.g. through cognitive/behavioral strategies. It therefore remains debatable whether patients will continue to exercise in the long term. Although there is still controversy concerning the effects of physical exercise on cardiovascular morbidity and mortality, it is certainly recommended as standard rehabilitation procedure for coronary patients, considering overall favorable psychosocial and physiological effects (Kugler et al., 1994). 
Table 3. Randomized controlled studies on interventions aimed at physical exercise in coronary patients.'

\begin{tabular}{|c|c|c|c|c|c|}
\hline Source & $\begin{array}{l}\text { Research question } \\
\text { /population }\end{array}$ & \multicolumn{2}{|c|}{ Intervention } & $N$ & Results \\
\hline $\begin{array}{l}\text { Kentala et } \\
\text { al, } 1972\end{array}$ & $\begin{array}{l}\text { Effect of physical } \\
\text { exercise in pattents } \\
\text { affer AMI }\end{array}$ & E. & $\begin{array}{l}\text { Physical exercise program, including risk } \\
\text { factor management, during } 12 \text { months } \\
\text { No intervention }\end{array}$ & 87 & $\begin{array}{l}24 \text { menthe } \\
\text { No significant differences berween } \mathrm{E} \text { and } \mathrm{C} \text { in total } \\
\text { mortality (both groups } 14 \% \text { ), cardiovascular mortality } \\
(10 \% \text { and } 12 \% \text {, respectuvely), or non-fatal recurrences } \\
(8 \% \text { and } 5 \% \text {, respectively). }\end{array}$ \\
\hline $\begin{array}{l}\text { Wilheimsen } \\
\text { et al., } 1975\end{array}$ & $\begin{array}{l}\text { Effect of physical } \\
\text { exercise in panents } \\
\text { after AMI }\end{array}$ & E & $\begin{array}{l}\text { Physical exercise program, including nsk } \\
\text { factor management, during } 48 \text { months } \\
\text { No interventon }\end{array}$ & $\begin{array}{l}158 \\
157\end{array}$ & $\begin{array}{l}48 \text { monthr: } \\
\text { No significant differences berween } E \text { and } C \text { in total } \\
\text { mortality ( } 18 \% \text { and } 22 \% \text {, respectrvely), cardiovascular } \\
\text { mortality ( } 18 \% \text { and } 21 \% \text {, respectuvely), or non-fatal } \\
\text { recurrences ( } 16 \% \text { and. } 18 \% \text {, respectively). }\end{array}$ \\
\hline $\begin{array}{l}\text { Kallio et al., } \\
1979\end{array}$ & $\begin{array}{l}\text { Effect of multuple rask } \\
\text { factor inter-vention in } \\
\text { patients after AMI }\end{array}$ & C & $\begin{array}{l}\text { Multifactorial intervention program } \\
\text { amed at health education and psycho- } \\
\text { social problems, including program } \\
\text { aimed at physical exercise, during 3-12 } \\
\text { months } \\
\text { No intervention }\end{array}$ & 187 & $\begin{array}{l}36 \text { monthi: } \\
\text { Significant difference between } E \text { and } C \text { in } \\
\text { cardhovascular mortality, in favor of the intervention } \\
\text { group }(19 \% \text { and } 29 \% \text {, respectively, } p<.05) \text {, no } \\
\text { significant differences in total mortality }(22 \% \text { and } 30 \% \text {, } \\
\text { respectively) and non-fital recurrences ( } 18 \% \text { and } 11 \% \text {, } \\
\text { respectively). }\end{array}$ \\
\hline
\end{tabular}

E: Expermental condition; C: Control condition

- table continues - 
Table 3 -continued

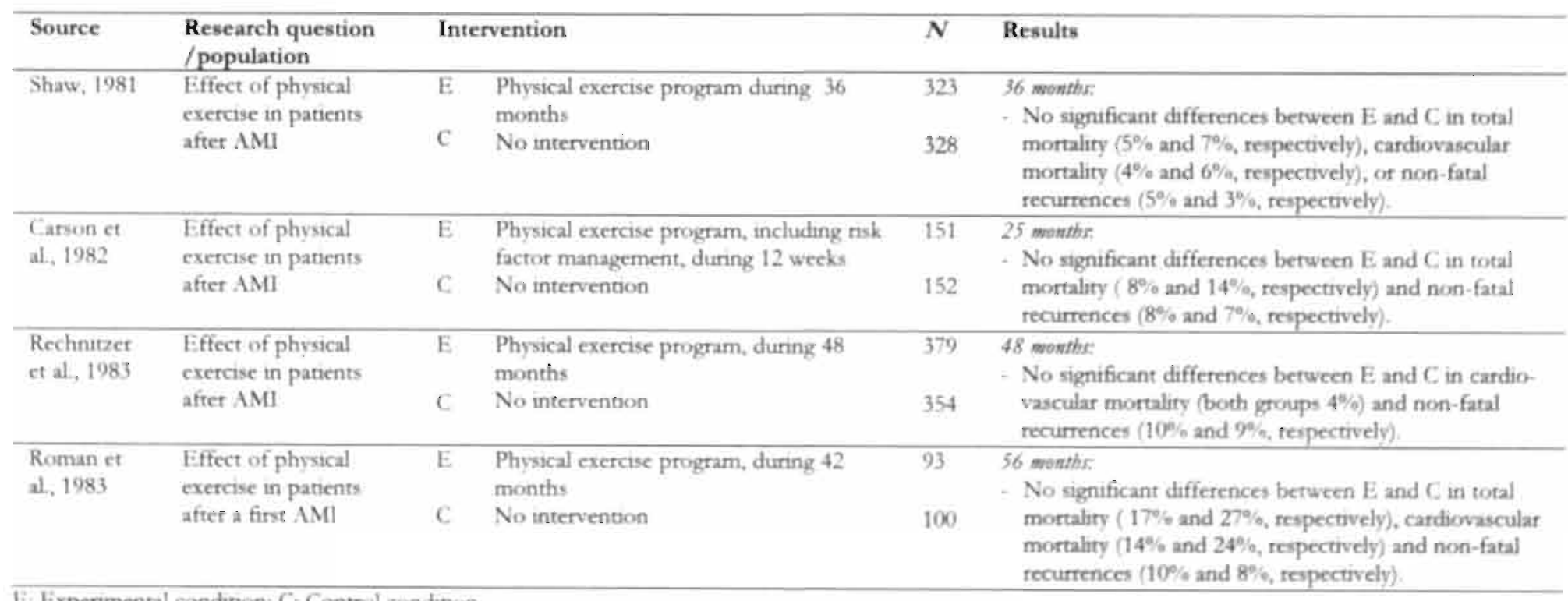

E: Experimental condinon; $\mathrm{C}_{-}$- Control condinon

- table continues . 
Table 3 - continued

\begin{tabular}{|c|c|c|c|c|}
\hline Source & Research question & Intervention & $\mathbf{N}$ & Results \\
\hline $\begin{array}{l}\text { Vermeulen } \\
\text { et al., } 1983\end{array}$ & $\begin{array}{l}\text { Effect of physical } \\
\text { exercase in patients } \\
\text { after a first AMI }\end{array}$ & $\begin{array}{l}\text { E Mulndisciplinary intervention animed at } \\
\text { physical exercise and other risk factors, } \\
\text { during } 6.8 \text { weeks } \\
\text { C No infervention }\end{array}$ & 51 & $\begin{array}{l}60 \text { monthe } \\
\text { No significant differences between } \mathrm{E} \text { and } \mathrm{C} \text { in } \\
\text { cardiovascular mortality ( } 4 \% \text { and } 10 \% \text {, respectively) } \\
\text { and non-fatal recurrences ( } 9 \% \text { and } 18 \% \text {, respectively). }\end{array}$ \\
\hline $\begin{array}{l}\text { World } \\
\text { Health Or- } \\
\text { ganizatuon, } \\
1984\end{array}$ & $\begin{array}{l}\text { Effect of physical } \\
\text { exercise in parients } \\
\text { after AMI }\end{array}$ & $\begin{array}{l}\text { E Eleven different programs ameed at } \\
\text { muluple risk factors and physical } \\
\text { exercise, during (varying per stuady) 6-36 } \\
\text { weeks } \\
\text { C No intervention }\end{array}$ & 705 & $\begin{array}{l}36 \text { months: } \\
\text { Two out of eieven studies showed significant } \\
\text { differences between } E \text { and } C \text { in total mortality } \\
\text { (Warsaw, } 3 \% \text { and } 18 \% \text {, respectively } p<.05 \text {; Ghent, } \\
18 \% \text { and } 4 \% \text {, respectively, } p<.01 \text { ); one study found a } \\
\text { significant difference in cardiovascular mortality } \\
\text { (Ghent, } 14 \% \text { and } 4 \% \text {, tespectively, } p<.05 \text { ), and rwo in } \\
\text { non-fatal recurrences (Brussels, } 13 \% \text { and } 2 \% \text {, } \\
\text { respectively, } p<.05 \text {; Hungary, } 9 \% \text { and } 0 \% \text {, } \\
\text { respectively, } p<.01 \text { ). }\end{array}$ \\
\hline $\begin{array}{l}\text { Marta et al, } \\
1985\end{array}$ & $\begin{array}{l}\text { Effect of physical } \\
\text { exercise in patients } \\
\text { after AMI }\end{array}$ & $\begin{array}{l}\text { E. Physical exercise program, during } 8.9 \text {. } \\
\text { weeks } \\
\text { C No intervention }\end{array}$ & 81 & $\begin{array}{l}55 \text { monthe } \\
\text { No significant differences between } E \text { and } C \text { in total } \\
\text { mortality (7\% and } 6 \% \text {, respectively), cardiovascular } \\
\text { mortality }(6 \% \text { and } 5 \% \text {, respectively) and non-fatal } \\
\text { recurrences }(6 \% \text { and } 11 \% \text {, respectively). }\end{array}$ \\
\hline
\end{tabular}

E- Expenmental condition: C: Control condition

table conntinues. 
Table 3 continued

\begin{tabular}{|c|c|c|c|c|}
\hline Source & $\begin{array}{l}\text { Research question } \\
\text { /population }\end{array}$ & Intervention & $\mathbf{N}$ & Results. \\
\hline $\begin{array}{l}\text { Kubo et al., } \\
1992\end{array}$ & $\begin{array}{l}\text { Effect of exercise trai- } \\
\text { nung on prevention of } \\
\text { estenosis in patients } \\
\text { after PTCA }\end{array}$ & $\begin{array}{l}\text { E Physical exercise program, during } 12 \\
\text { weeks } \\
\text { C No intervention }\end{array}$ & $\begin{array}{l}18 \\
20\end{array}$ & $\begin{array}{l}3 \text { montbs: } \\
\text { Significant difference in progression of stenosis } \\
\text { between } E \text { and } C(82 \% \text { and } 120 \% \text {, respectuvely, } p< \\
05) \text {. } \\
\text { No significant difference in proportion of partents } \\
\text { with restenosis between } E \text { and } C(17 \% \text { and } 40 \% \text {, } \\
\text { respectively). }\end{array}$ \\
\hline $\begin{array}{l}\text { Hambrecht } \\
\text { et al., I993 }\end{array}$ & $\begin{array}{l}\text { Effect of physical } \\
\text { acturity on cardio- } \\
\text { respiratory fitness and } \\
\text { progresston of } \\
\text { coronary athero. } \\
\text { sclerusis in patients } \\
\text { with CAD }\end{array}$ & $\begin{array}{l}\text { E Physical exercise program during } 12 \\
\text { months, and low-far diet } \\
\text { C No untervention }\end{array}$ & $\begin{array}{l}29 \\
33\end{array}$ & 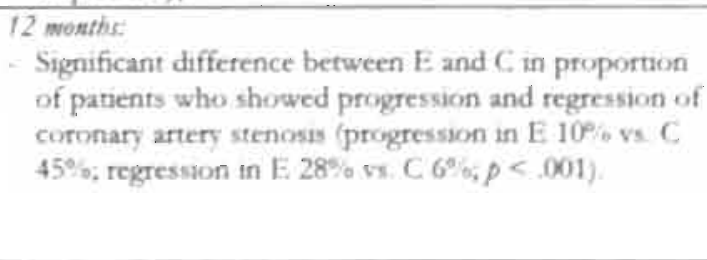 \\
\hline
\end{tabular}

F. Experumental crindition; C. Control condinon 


\section{TYPE A BEHAVIOR}

In the etiology of CHD the role of the type A behavior pattern as a potential risk factor has received considerable attention. The Western Collaborative Group Study was the first large prospective study that confirmed the association between this behavior pattern, characterized by a continuously harassing sense of time urgency and easily aroused or 'free-floating' hostility, and elevated risk of CHD. Type A behavior was associated with a two- to threefold increased risk for AMI compared with the type B pattern, which was defined as the relative absence of type A characteristics (Rosenman et al., 1975). This positive association with incidence of CHD was also found in a substantial number of prospective and casecontrol studies throughout the world (Appels, Jenkins, \& Rosenman, 1982; Matthews \& Haynes, 1986). Moreover, global type A behavior has been found to predict recurrent coronary incidents (Brackett \& Powell, 1988; Jenkins, 1976; Matthews \& Haynes, 1986).

In contrast to the consistent and often strong relationships reported before 1985, subsequent findings have raised questions about this relationship, as some studies failed to find strong associations between global type A behavior and CHD (Booth-Kewley \& Friedman, 1987; Matthews, 1988). The studies that found no associations were, however, frequently characterized by high-risk study populations, i.e. patients with known atherosclerotic disease, in contrast to healthy population studies. This restriction of range may lead to a decrease in magnitude of the associations between CHD and type A behavior (Miller, Turner, Tindale, Posavac, \& Dugoni, 1991). Moreover, nonsignificant findings most often occurred in studies using self-report measures of type A behavior, such as the Jenkins Activity Survey or the Bortner Rating Scale. It is now well-established that these self-rating scales show only low correlations with CHD, in contrast to the Structured Interview [SI] for measuring type A behavior (Miller et al., 1991). These conflicting findings have cast serious doubts on the validity of type A behavior as a risk factor for CHD. Indeed, in the original Western Collaborative Group Study it was shown that in survivors of AMI, type $A$ behavior was associated with a lower risk of subsequent CHD mortality over 22 years of follow-up (Ragland \& Brand, 1988). Therefore, recent studies have emphasized the importance of studying the different components of type $A$ behavior. These showed that particularly the 'hostility' component is associated with increased risk of CHD (Miller et al., 1996; Smith, 1992). The 'time-urgency' component should, however, not be disregarded in the diagnosis of type A behavior as contributing to increased CHD risk (Fricdman \& Ghandour, 1993).

The physiological mechanisms by which stress and type $\mathrm{A}$ behavior may affect CHD are increasingly becoming clear. There are strong implications that persons exhibiting type A behavior may habitually react to stress with an exaggerated response of the sympathetic nervous system, leading to chronic increases in catecholamine secretion (Schneider, 1994). Excessive adrenergic activity could possibly contribute to CHD through several mechanisms (McEwen, 1998), one of 
which is that platelet-aggregation is stimulated, eventually leading to occlusive thrombus formation in susceptible individuals (Levine et al., 1985). That psychological stress may indeed potentiate platelet activation was found by Markovitz et al. (Markovitz, Matthews, Kiss, \& Smitherman, 1996). Moreover, this effect seems to be modulated in part by personality traits associated with CHD: Type A behavior, and in particular hostility, was related to greater platelet reactivity during acute stress (Markovitz et al., 1996). Evidence has also been found of increased cardiovascular and neurohormonal reactivity among type A's, especially males, to various laboratory tasks, or even chronic elevation of catecholamine secretion on laboratory tasks (Williams, Suarez, Kuhn, Zimmerman, \& Schanberg, 1991).

\section{Secondary prevention trials for coronary patients: modifying type A behavior}

Studies in employed, healthy populations have shown that type $\mathrm{A}$ behavior (and its components) may be modified (Gill et al., 1985; Suinn \& Bloom, 1978). A metaanalysis of 18 controlled studies indicated that type $\mathrm{A}$ behavior may be modified in coronary patients as well (Nunes, Frank, \& Kornfeld, 1987). This analysis suggested that interventions in which a combination of treatment techniques was used, thus addressing a number of different facets of the behavior pattern, may be most effective in reducing type A behavior. Moreover, treatment of the type A behavior pattern was associated with a reduction in coronary events of about $50 \%$; being based on only two studies, however, this finding should be treated with caution. To a certain extent, the findings of this meta-analysis were weakened by measurement problems. Most instruments were self-report measures of type A behavior that might reflect an experimenter expectancy effect instead of true change, and only a few studies used measures that were validated predictors of CHD, such as the original Structured Interview (SI) and the Videotaped Structured Interview (VSI). The use of validated predictors would seem most important for the evaluation of treatments aimed ultimately at modification of clinical CHD.

The Recurrent Coronary Prevention Project (RCPP) was the first and as yet largest trial that studied whether type A behavior could be modified, and what effects this might exert on subsequent cardiovascular morbidity and mortality rates (table 4) (Friedman et al., 1984; Friedman et al., 1986; Friedman et al., 1982). At 4.5 years follow-up, reductions in global type $\mathrm{A}$ behavior as well as in its components were significantly larger in the type A-intervention group as compared to the control group. This reduction was dose-dependent, such that an increasing number of creatment contacts was associated with increasing change. Moreover, the cardiac recurrence rate was significantly less in the experimental group receiving type $A$ counseling. The reductions in type $\mathrm{A}$ behavior and cardiac recurrence appeared to be maintained 1 year after cessation of behavioral counseling. Moreover, when subjects originally randomized to the control group were exposed to 1 year of type

"Table 4 contans only studes that used the ongral SI and the VSI to measure type A behavior 
A behavioral counseling after the original study was ended, a significant decrease both in the intensity of their type $\mathrm{A}$ behavior and in the rate of cardiac recurrence appeared to occur during this same year (Friedman et al., 1987).

Patients undergoing type A counseling in the RCPP showed significant reductions not only in type A behavior, but also in depression, and increases in self-efficacy and social support. An increasing number of treatment contacts in the type A counseling group was associated with larger changes in these psychosocial outcomes. Thus, reduced cardiac recurrences in the experimental group may partly be explained by changes in psychosocial factors, and may not exclusively be attributed to type A behavior modification (Mendes de Leon, Powell, \& Kaplan, 1991).

In a Swedish study it was found that an intervention aimed at type $A$ behavior modification and based on the RCPP model, was associated with significant reductions of type A behavior in treated patients. (Burell et al., 1994). These results were maintained at 1-year follow-up. Treated patients also tended to have fewer cardiac recurrences than control patients. Reduced cardiovascular morbidity and mortality was also found exclusively in post-CABG patients, after participation in a comparable type A behavior intervention program (Burell, 1996).

Thus, not many studies were carried out on type $A$ behavior modification in coronary patients. The few studies that used the SI and the VSI (the only measures that proved valid for measuring type $A$ behavior), all showed that this behavior pattern is amenable to changes. Although in one of the trials the study population was rather small (Burell et al., 1994), indicating that the results should be interpreted with caution, all three trials showed a reduction of recurrent cardiac events after type $A$ behavior modification. All trials studied a comparable type of intervention, using a diversity of cognitive/behavioral techniques to realize the behavior changes. The major drawback of these programs, however, was that they were lengthy ( $\geq 1$ year), and therefore not easily applicable in general health care services. Moreover, the intensity of these programs may only attract highly motivated patients, leaving large groups of patients untreated. Still, the positive results concerning reduced recurrences warrant treatment of type $A$ behavior. 
Table 4. Randomized controlled studies on non-pharmacological interventions aimed at reduction of type A behavior in coronary patients.

\begin{tabular}{|c|c|c|c|c|c|}
\hline Source & Research & \multicolumn{2}{|c|}{ Intervention } & \multirow{2}{*}{$\begin{array}{l}\mathbf{N} \\
592 \\
\end{array}$} & $\begin{array}{l}\text { Results } \\
\text { yoar: } \\
- \text { Significant difference in favor of } \mathrm{E} \text { in rates of nonfatal } \\
\text { infarction }(1 \% \text { in } \mathrm{E} \text { vs. } 3 \% \text { in } \mathrm{C}, p<.05) \text {. } \\
3 \text { yors }\end{array}$ \\
\hline $\begin{array}{l}\text { Frnedman er al. } \\
1982,1984 . \\
1986\end{array}$ & $\begin{array}{l}\text { Possibiliry of } \\
\text { altering type } A \\
\text { behavior in patients } \\
\text { affer AMI, and the } \\
\text { effect of such } \\
\text { alferation on car- } \\
\text { diovascular mor- } \\
\text { bidity and mortality }\end{array}$ & C & 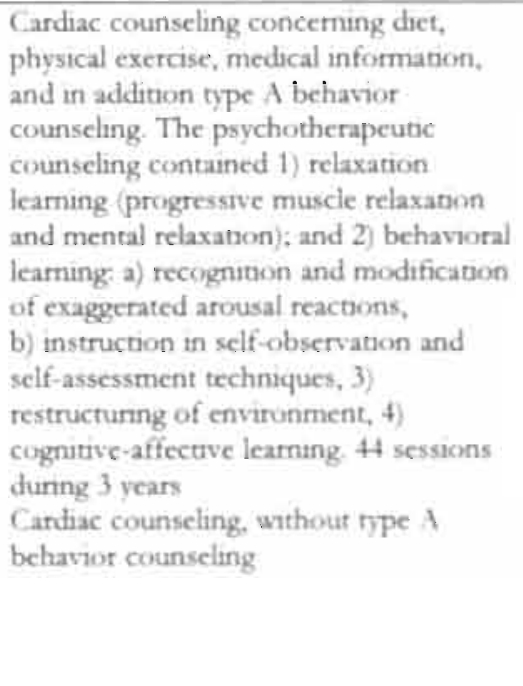 & & 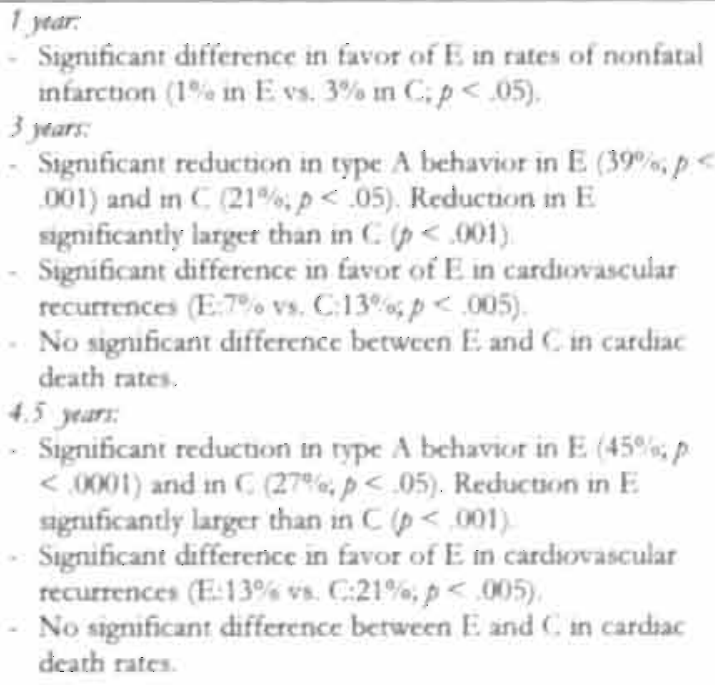 \\
\hline
\end{tabular}

F. Expermental condinon: C: Contrul cundition 
Table 4 - continued

\begin{tabular}{|c|c|c|c|c|c|}
\hline \multirow{2}{*}{$\begin{array}{l}\text { Source } \\
\text { Burell et al., } \\
\text { I994 }\end{array}$} & \multirow{2}{*}{$\begin{array}{l}\text { Research } \\
\text { question/ } \\
\text { population } \\
\text { Effects of type A } \\
\text { behavior } \\
\text { modification in } \\
\text { patients after AMI }\end{array}$} & \multicolumn{2}{|c|}{ Intervention } & \multirow{2}{*}{$\begin{array}{l}\mathbf{N} \\
25\end{array}$} & \multirow{2}{*}{$\begin{array}{l}\text { Results } \\
\text { 1)ear: } \\
\text { Significantly larger reduction of type } A \text { behavior }(p< \\
02) \text { in } E \text { than in } C^{2} \\
\text { Reduced cardiovascular recurrence rate in E comparec } \\
\text { with } C(p<.05), 2\end{array}$} \\
\hline & & C & $\begin{array}{l}\text { Cardiac counseling in healthy life-style, } \\
\text { nsk factors, medical informatoon, and in } \\
\text { addition type A behavior counseling; } 35 \\
\text { sessions durng } 1 \text { year } \\
\text { Cardiac counseling, withour type A } \\
\text { behavior counseling. }\end{array}$ & & \\
\hline Burell, 1996 & $\begin{array}{l}\text { Effect of } \\
\text { modification of } \\
\text { type } A \text { behavior } \\
\text { and psychosocial } \\
\text { nsk factors in } \\
\text { CABG patients }\end{array}$ & C & $\begin{array}{l}\text { Behavioral intervention aimed at re- } \\
\text { duction of type } A \text { behavior and psycho- } \\
\text { social risk factors; } 17 \text { sessions during } 1 \\
\text { year, followed by } 5-6 \text { booster sessuons in } \\
\text { the } 2 \text { nd and } 3 \text { rd year } \\
\text { No intervention }\end{array}$ & 128 & $\begin{array}{l}\text { 5.6.5 years: } \\
\text { Significant reduction in type A behavior in E, not in } C \\
(p<.0003) \text {. } \\
\text { Significantly lower rate of cardiovascular recurrences } \\
\text { in } \mathrm{E} \text { than in } \mathrm{C}\left(11^{\circ} \% \text { and } 14^{\circ} \% \text {, respectively, } p<.05\right) \text {. } \\
\text { - Significantly lower total mortality rate in } \mathrm{E} \text { than in } \mathrm{C} \\
\left(5 \% \text { and } 12^{\%} \% \text {, respectuvely; } p=02 \text {. }\right.\end{array}$ \\
\hline
\end{tabular}

E: Experimental condition; C: Control condition

2 percentage not reported in artacle 


\section{DISCUSSION}

In order to study the effects of risk factor modification by means of nonpharmacological interventions in patients suffering from CHD, we scrutinized every risk factor separately. Each factor was amenable to modification through interventions aimed at behavior change. Behaviorally oriented interventions, incorporating cognitive and behavioral smoking cessation techniques, showed positive results in reducing smoking rates in AMI patients (Ockene et al., 1992; Taylor et al., 1990). Less favorable results were found for CABG patients (Rigotti et al., 1994). Elevated serum cholesterol concentrations were reduced by intensive dietary modifications. Thus, major changes in dietary habits can be achieved in CHD patients, and this may even lead to stabilization or regression of coronary atherosclerosis. These favorable effects may not solely be attributed to the adoption of a low-fat diet, since in some interventions additional components were included, such as stress-management or exercise training (Omish et al., 1990; Ornish et al., 1983). Several studies indicated that physical exercise may prevent progression of atherosclerosis as well. Secondary prevention programs aimed at increasing physical exercise seem to bring about reduced cardiovascular mortality and morbidity, although unambiguous evidence concerning these effects is still lacking. Finally, modification of type A behavior is possible through interventions based on multiple cognitive-behavioral techniques. Furthermore, reduction in type A behavior may lead to reduced morbidity in AMI and CABG patients.

Only a few randomized, controlled studies have been carried out that focused primarily on changing one risk factor only. Moreover, trials that met our inclusion criteria were often directed at changing several other risk factors as well. In addition, trials often used a multimodal approach to the modification of health behaviors, integrating a number of cognitive-behavioral methods within one intervention. Overall, this seriously limits firm conclusions concerning the relative contributions of each additional component to the study effects. Moreover, trials were often relatively small, especially the studies on cholesterol reduction, so prudence is called for in the interpretation of results:

Still, from this review several conclusions can be drawn. Most importantly, behavioral modification of each separate risk factor may add considerably to usual care in coronary patients. It is clear, however, that risk factors of CHD are additive in their effects and considerable attention must therefore be paid to all important risk factors. rather than concentrating on a single factor such as smoking. Factors that confer the highest relative risk in a given patient should have priority. Thus, programs focused on modification of multiple risk factors may be expected to be more effective. Convincing evidence in this direction was shown in a recent metaanalysis (Linden, Stossel, \& Maurice, 1996).

A further conclusion is that in selected patients endowed with exceptional motivation, impressive changes in cardiovascular status are feasible if comprehensive lifestyle changes are made. This was particularly clear in the study by Niebauer et al. (Niebauer et al., 1997), where documented regression of 
coronary narrowing occurred in patients who were willing to exercise 5 to 6 $\mathrm{h} /$ week, as well as in. the Lifestyle Heart Trial (Ornish et al., 1990; Ornish et al., 1998). Although the latter was a randomized study, patients were asked to take part only after they had been randomized to either the control or the experimental groups. Drop-out rates were rather high: $47 \%$ of the experimental group and $58 \%$ of the control patients refused to participate. It is conceivable that the remaining patients were highly motivated to make certain lifestyle changes. Scherwitz et al. observed that patients in the intervention group were indeed primarily highly motivated, well-educated coronary patients (Scherwitz et al., 1995). So these encouraging findings may not be easily translated to the general population of coronary patients. It therefore remains a challenge for behavioral medicine to develop enduring motivational strategies with these patients. One important question raised by these trials is whether so radical a lifestyle change is actually necessary to delay the progression of coronary atherosclerosis. Less comprehensive changes might have somewhat less favorable, but still positive, effects, and may be acceptable for many more patients. Moreover, even modest reductions of serum cholesterol lead to significant reductions in cardiovascular morbidity or mortality (Scandinavian Simvastatin Survival Study Group, 1994). The Lifestyle Heart Trial, however, showed that clinically significant regression of coronary atherosclerosis is feasible only if lifestyles are changed radically; patients with the best adherence showed the most regression, in particular after five years of follow up (Ornish et al., 1990; Ornish et al., 1998). The attitude of someone willing to make comprehensive lifestyle changes may often be quite different from that of someone preferring invasive procedures (i.e., PTCA, CABG). The decision to make comprehensive lifestyle changes requires commitment, discipline and willingness to assume personal responsibility for one's health (Ornish, 1998).

In spite of the largely favorable results of all reviewed studies, some trials did not show unequivocal significant reductions in cardiovascular mortality or morbidity. Although the patients' life quality was not an outcome variable that we focused on, in our opinion it is equally important to also study improvements in patients' wellbeing and life quality as possible effects of non-pharmacological interventions. It is well known that while quality of life may improve, changes in morbidity or mortality cannot always be shown. Several other psychological outcomes of nonpharmacological interventions should be studied as well, in particular depression and vital exhaustion. Not only are depression and vital exhaustion frequently mentioned consequences of AMI, they have also been shown to adversely affect prognosis in patients with CHD (Ford et al., 1998; Frasure-Smith et al, 1995; Kop et al., 1994). These mood states are increasingly being recognized as important targets for intervention, both pharmacological and cognitive/behavioral (Rozanski, Blumenthal, \& Kaplan, 1999).

The lack of significant reductions in cardiovascular mortality or morbidity in some trials may partially be explained by the relatively small-size study populations; these simply may have been. too small to detect any clinically significant differences. Behavioral intervention. studies generally are of necessity labor-intensive and most often long-lasting, which raises questions about the feasibility of this approach on 
a larger scale. Moreover, these evaluation studies are dependent on disease incidence, as a result of which the number of subjects under study is often unintentionally small. In this case, meta-analysis may offer a solution; significant effects may be detected that are otherwise not demonstrable as such in each single study. The wide divergence of methods of intervention makes meta-analyses less valid, however. Another problem often encountered with intervention studies is selective drop-out; the experimental group may thus represent primarily highly motivated patients as in the Omish trials, because the less motivated drop out before the end of intervention.

It should be emphasized that although some studies did not find significant overall effects, they often do show favorable effects within specific patient subgroups. Most studies focused on the mean effects of one particular rehabilitation program. This way, individual differences in effects may be lost. Sub-analyses may show these effects; for example, a smoking cessation program that was not effective for the entire group of coronary patients showed positive results for patients with severe AMI (Ockene et al., 1992).

Thus, to be maximally effective, risk management should focus on selection of patients most likely to benefit from a particular program, or even better, patients should be referred to such treatment which is most appropriate for their needs. This is in accordance with recent general guidelines for cardiac rehabilitation as formulated in Great Britain, the Netherlands, and other European countrics, which emphasize determination of the patients' needs, and individual subgoals for rehabilitation (British Association for Cardiac Rehabilitation, 1995; Nederlandse Vereniging voor Cardiologie en de Nederlandse Hartstichting, 1995). In theory, the most suitable type of cardiac rehabilitation should be chosen, but in actual practice, the needs of individual patients are still barely taken into account. To optimize the effects of cardiac rehabilitation, every patient should be screened to determine which risk factors contributed to his or her condition, after which the most suitable cardiac rehabilitation program should be applied. Very likely this approach will improve the patients' motivation, which could have a favorable influence on the effects of the intervention. It was shown that exceptionally motivated patients make comprehensive lifestyle changes which lead to improved cardiovascular status. In order to reach the majority of patients, better applicable, though effective behavioral interventions should be developed. In particular, the motivation to sustain acquired lifestyle changes will have to be reinforced by noticeably favorable effects, which ought to be larger with more intensive changes. Whatever the patient decides, the cardiologist and psychologist should support this decision and explain the relative risks, benefits, costs and side effects of each secondary prevention, approach, be it invasive or non-invasive, pharmacological or nonpharmacological (Ornish, 1998). Therefore, new research should focus on maintaining motivation or the lack thereof in patients, as well as on the modification of this latter attitude, e.g. by scrutinizing the quality of information that parients receive from cardiologists and psychologists. In the final analysis, lack of motivation most often leads to relapse and impaired prognosis. 



\section{CHAPTER 2}

PSYCHOLOGICAL EFFECTS OF A SHORT BEHAVIOR MODIFICATION PROGRAM IN PATIENTS WITH ACUTE MYOCARDIAL INFARCTION OR CORONARY ARTERY BYPASS GRAFTING

Sulmitted in rrvised form as:

Sebregts, E.H.W.J., Falger, P.R.J., Appels, A., Kester, A.D.M., Bar, F.W.H.M. Psychological effects of a short behavior modification program in patients. with acute myocardial infarction or coronary artery bypass grafting. $A$ randomized controlled trial. 


\section{ABSTRACT}

The effects of a short intervention on behavioral risk factor modification in patients with coronary artery disease on type A behavior, vital exhaustion, and depression were studied. Acute myocardial infarction patients or patients who underwent coronary artery bypass grafting were randomly assigned to an eightweek multiple risk modification group program $(n=94)$ or a control group $(n=$ 90), that received usual care with standard physical exercise training. Patients were assessed before intervention, directly after intervention and at 9-month follow-up. The intervention was effective in reducing hostility and total type $A$ behavior at postintervention $(p=.01)$ and at 9 -month follow-up $(p=.03)$. The intervention had no overall impact on vital exhaustion. For patients with high baseline scores of vital exhaustion, however, the intervention reduced vital exhaustion at 9-month follow-up compared to controls $(\phi=.03)$. No main effects were found for depression measured by the Beck Depression Inventory, whereas we unexpectedly found that the percentage of patients with major depression was reduced in the control group, but not in the intervention group. The results indicate that a short behavioral intervention for coronary patients can result in relatively large and persistent reductions in some psychological risk factors. Stronger effects might be achievable in more distressed study populations. In view of the negative findings on diagnosis of depression, we do not unequivocally advise the intervention to the general population of AMI and CABG patients. 


\section{INTRODUCTION}

Coronary artery disease (CAD) is the leading cause of death in most industrialized Western. countries. Many psychological factors have been linked to CAD, as they may be detrimental consequences of the disease, or because they may exert an additional risk for the CAD patient. The significance of intervening in psychological risk factors is elucidated by several recent studies that have stressed the influence of psychosocial risk factors on stabilizing underlying cardiovascular pathophysiology (Hemingway \& Marmot, 1999; Rozanski et al., 1999). Prognosis of patients with CAD may be adversely affected by vital exhaustion and depression (Frasure-Smith et al., 1995; Kop et al., 1994), whereas the type A behavior pattern is another psychological factor that has been linked to CAD (Brackett \& Powell, 1988; Jenkins, 1976; Matthews \& Haynes, 1986; Rosenman et al., 1975). Several studies reported epidemiological uncertainties regarding type A behavior as being an independent risk factor for recurrent AMI (Ragland \& Brand, 1988; Smith, 1992). Nevertheless, the attention, paid. to this behavior pattem in rehabilitation programs seems legitimate, since several clinical trials have demonstrated that an intervention on type A behavior after AMI reduces the risk of a new coronary event (Burell, 1996; Burell et al., 1994; Friedman et al., 1986). Moreover, reducing type A behavior may lead to a decrease in psychological distress factors related to CAD, particularly vital exhaustion. In previous reports, it was postulated that vital exhaustion might reflect a. breakdown in adaptive mechanisms to prolonged and uncontrollable psychological stress (Appels, 1990; Appels \& Mulder, 1989; Falger, 1989). Type A behavior, a style of behavior characterized by a continuously harassing sense of time urgency and easily aroused hostility, has been suggested to increase the risk of becoming exhausted.

Cardiac rehabilitation trials that showed clear positive results in reducing type $A$ behavior and psychological distress, and in reducing cardiovascular morbidity, are comprehensive and lengthy (Burell et al., 1994; Friedman et al., 1986). Such intensive time-consuming programs are not easily applicable in general health care settings. Therefore, we intended to develop a relatively short intervention program of eight weeks, accessible for a large group of AMI and Coronary Artery Bypass Grafting (CABG) patients. Although many studies that demonstrated favorable effects of psychosocial interventions were aimed at modification of one risk factor in particular (Sebregts, Falger, \& Bär, 2000), our intervention explicitly addressed multiple modifiable risk factors. It was directed at the reduction of psychological stress / distress (type A behavior in particular), reduction of excessive consumption of dietary fat, elevated serum cholesterol, lack of physical excrcise, and - although less explicitly - smoking and insufficient social support through involvement of the patients' partners in the intervention (Falger, Sebregts, Van Leuteren, \& Bär, 2000). ${ }^{1}$

Effects of the intervenuon on improving dietary habits and cholesterol are described in chaptet 3 
The present paper presents the psychological effects of this intervention program. We tested the hypothesis that the intervention reduces $A$ behavior, vital exhaustion and depression.

\section{METHODS}

\section{Participants}

All patients admitted to the University Hospital Maastricht, in Maastricht, the Netherlands, during the period of February 1996 until November 1997 were identified as eligible for the study by a team of physiotherapists working at the hospital's physiotherapy department. These patients were referred to them by cardiologists for physical training following AMI or CABG. Patients included were less than 70 years of age, who were admitted to the University Hospital Maastricht with a confirmed diagnosis of AMI, CABG, or both, and who were able to participate in the regular physiotherapy exercise-program starting early after discharge at the hospital. Two hundred patients were to be included in the study, a number that was based on feasibility within the time limits of the study. Patients were excluded if they were non-Dutch speaking, illiterate, or if they were currently suffering from any psychiatric disorder that would severely disturb participation in the intervention.

\section{Procedure}

After informed consent was given, patients were randomly assigned to either the intervention $(n=106)$ or a control group $(n=98)$. A stratified randomization procedure was developed by a person not further involved in the study to allocate men and women to the intervention or control group. The outcome of the randomization was put in a sealed envelope, and patients received this envelope after the baseline interview. Both groups (intervention and control) received usual. medical care. For patients that were assigned to the intervention, the intervention started within 3-8 weeks after discharge.

Psychological assessments for the intervention group were performed (1) at baseline before the beginning of the intervention, (2) 8 weeks later immediately after the intervention, and (3) 9 months after termination of the intervention, which was approximately one year after admission to the hospital. Measures for control patients were collected at comparable moments in time. Psychological measures consisted of interviews and self-report questionnaires. The interviewers (four in total) remained unaware of patient group assignment. All interviews were recorded on videotape. Interviewers received an intensive training in conducting and scoring the interviews. 


\section{Usual care}

Both the control group and the intervention group received the same usual medical care, consisting of regular check-ups by a cardiologist who was blinded to group allocation, and they all had postdischarge exercise training sessions. In the Netherlands, exercise training is part of the usual care for patients with AMI or $\mathrm{CABG}$. Patients received standardized exercise training 3 times a week during 6 weeks.

During the regular medical check-ups, standard usual care was offered, comparable to the care patients received. who did not participate in the study. This meant that the cardiologist systematically checked the clinical history, performed physical examination, a 12-lead ECG recording was done and if appropriate, blood tests were performed. Titration of drugs was performed until clinical results were satisfactory. The cardiologist provided concise information on risk modification, e.g., patients were advised to eat a low-fat diet. For each AMI patient exercise tests were performed according to the Bruce Protocol during hospitalization, and one year after discharge. The CABG patients only had an exercise test one year after discharge.

\section{Intervention}

In addition to the usual care described above, patients allocated to the intervention were offered a combined stress-management and health education program during eight weekly 2.5 -hr sessions. Table 1 presents the outline of the program. The contents of the intervention were primarily based on the methods of three major long-term clinical trials, the Recurrent Coronary Prevention Project (Bracke \& Thoresen, 1996), Project New Life (Burell, 1996), and the I.ifestyle Heart Trial (Ornish et al., 1990), adapted to a format that would be feasible within the context of a short intervention program. During the sessions, 6 to 10 cardiac patients and their partners (whose participation was encouraged) were informed about CAD risk factors and risk factor modification by a multidisciplinary team (table 1). The first hour-and-a-half consisted of didactic presentations of these issues, including advice and/or exercises to actively modify each participant's CAD risk factor profile. In the remainder of each session participants were encouraged to report their experiences with the issues at hand. Each session was concluded with breathing and relaxation exercises based on the work of Van Dixhoom (Van Dixhoom, 1997). The purpose of these exercises was primarily to increase the patients' sense of relaxation and body awareness. Patients were given the opportunity to take an audiocassette tape home with them to practice these breathing and relaxation exercises. At the end of most sessions, patients were given homework assignments, as well as written information, with reference to the specific topics mentioned above. All sessions were led by a psychologist, and exther a social worker or a pastor was present as cotherapist. After the last session, three follow-up sessions were scheduled at 3,6, and 9 months, in order to discuss the achievement patients had made with respect to risk factor modification. 
Table 1. Contents of the intervention sessions

\begin{tabular}{|c|c|}
\hline Session & Contents \\
\hline 1. & $\begin{array}{l}\text { Explanation of the aim of the intervention. An inventory is made of the } \\
\text { patients' histories and risk factors (psychologist). }\end{array}$ \\
\hline 2. & The concepts of atherosclerosis, AMI and CABG (cardiologist). \\
\hline 3. & $\begin{array}{l}\text { Healthy diet (dietitian). Patients are given advice to eat a low-fat diet, how to } \\
\text { do so, and the health benefits of radical low-fat dietary changes are pointed out } \\
\text { to them. Special attention is pard to the favorable effects of a vegetarian diet. }\end{array}$ \\
\hline 4. & Physical exercise (physiotherapist). \\
\hline 5. & $\begin{array}{l}\text { Type A behavior/hostility and stress: } 1 \text { (psychologist). Strategies are discussed } \\
\text { for modification of type A behavior. Signs and symptoms of type A behavior } \\
\text { are presented, together with epiderniological evidence about the parhogenicity } \\
\text { of type A behavior. Major behavioral drills from the Recurrent Coronary } \\
\text { Prevention Project are discussed. }\end{array}$ \\
\hline 6. & $\begin{array}{l}\text { Type A behavior/hostility and stress: II (psychologist). The concept of chronic } \\
\text { sympatheric hyperarousal as major pathophysiological process underlying type } \\
\text { A behavior is introduced. Health-promoting coping strategies with life stressors } \\
\text { and daily hassles, and prevention of anger, hostulity and aggression are } \\
\text { discussed and practuced. }\end{array}$ \\
\hline 7. & Personal values and suctal support systems (pastor). \\
\hline 8. & $\begin{array}{l}\text { Evaluation of the program and identification of problems in the process of } \\
\text { behaver change (psychologist). Contribution of general practitioner to } \\
\text { recovery process and behavior change (general practitioner). }\end{array}$ \\
\hline
\end{tabular}

\section{Psychological measures}

Type A behavior was measured using a Dutch version of the Videotaped Structured Interview (VSI) (Friedman \& Powell, 1984). The VSI covers three principal components of type $A$ behavior: hostility, time urgency and insecurity. An interviewer notes the content of a participant's responses to 32 questions, and also observes and records specific psychomotor and physiologic manifestations diagnostic of type A behavior (e.g., tense posture, head nodding when interviewer speaks, interruption of the interviewer). For practical reasons it was not possible to score psychomotor characteristics for the total study population. Instead, videotaped interviews of a random sample of 50 patients were reviewed to score psychomotor, behavioral and physiologic manifestations of type A behavior at baseline and 9-month follow-up.

I ital exbaustion was measured by the Maastricht Interview for Vital Exhaustion (MIVE) (Meesters \& Appels, 1996a; Meesters \& Appels, 1996b). The interview contains 23 items that can be answered by either yes (1 point) or no ( 0 points). A score of $\geq 8$ is considered positive for vital exhaustion.

Depressed wood state was assessed using a structured interview according to the Diagnostic and Statistical Manual of Mental Disorders (third edition, DSM-IIIR) classification of Major Depressive Disorder (Structured Clinical Interview for DSM-III-R, or SCID).

In addition to the interview, the Beck Depression Inventory (BDI) was administered (Beck, Rush, Shaw, \& Emery, 1979). The BDI contains 21 items, 
covering symptoms and atritudes displayed frequently by depressed patients. Scores $<10$ represent none or minimal depression; 10-18 mild to moderate depression; 19-29 moderate to severe depression; 30-63 severe depression (Beck, Steer, \& Garbin, 1988).

\section{Clinical data}

Clinical data, used for baseline comparisons of the intervention and control group, were collected by a cardiologist from the University Hospital Maastricht, who was blinded to the patients' group allocation. Structured inventories were filled out, which contained items concerning the patient's medical history (obtained from the medical files), angina pectoris, dyspnea, enzymatic infarct size during hospitalization, medication, and echocardiographic as well as angiographic data, if available. In addition, during follow-up cardiac complaints and cardiac or other events were recorded.

\section{Statistical analyses}

Analyses were performed according to the intention-to-treat principle, meaning that patients were analyzed in the original groups formed by randomization. Effects of the intervention on psychological outcome measures were assessed by means of analyses of covariance (ANCOVA), using the baseline scores as a covariate to correct for differences in scores at baseline between the control and intervention group. In addition, for each of the psychological outcome measures, the variables gender, age, and 'AMI (+ CABG) versus CABG only' were used as preplanned covariates in the analyses. This last variable was included because of the possible influence an AMI experience might have on the psychological outcome measures. Logistic regression analyses were used to compare the intervention and control groups on SCID depression. Separate analyses were performed for effects at postintervention and 9-month follow-up.

After these main analyses, an interaction variable Group Allocation $x$ Bascline Score was included in the ANCOVAs to check whether intervention effects were different for patients with high or low baseline scores on the psychological variables. If the interaction variable was significant, subgroup analyses were performed for patients with high or low baseline scores.

\section{RESULTS}

\section{Participants and baseline characteristics}

Figure 1 shows a flowchart of the patients approached for participation in the study. 204 patients gave signed informed consent, which was $67 \%$ of all eligible patients. One hundred patients refused to participate for the following reasons: work or too busy $(17 \%)$, no need, or knows how to make lifestyle changes without help $(17 \%)$, visiting the hospital too often, it will be too much $(12 \%)$, not 
motivated to make changes $(6 \%)$, personal or health problems $(6 \%)$, partner does not take part $(5 \%)$, does not want any obligations $(4 \%)$, prefers not to be confronted with disease $(4 \%)$, too shy to take part in group intervention $(2 \%)$, does not want to hear other patients sorrows $(2 \%)$, other $(5 \%)$, unknown $(20 \%)$, Compared to participants, nonparticipants were more often female (participants $13.7 \%$ female vs. nonparticipants $33.0 \% ; p=.00$ ). There were no differences between participants and nonparticipants in mean age (55.6 vs. 57.3 , respectively; $p$ $=.13$ ) and cardiac incidence for which they were hospitalized (participants $69.1 \%$ AMI, 7.8\% AMI + CABG, 23.0\% CABG; nonparticipants $73.0 \%$ AMI, $5.0 \%$ AMI $+\mathrm{CABG}, 22.0 \% \mathrm{CABG} ; p=.62$ ).

In spite of considerable efforts to reduce missing data to a minimum, dropouts could not be avoided. Of all 204 patients included in the study, 20 dropped out, reducing the total number of patients in the study to 184 . Since they dropped out before baseline measurement - the time at which patients received their group allocation, this cannot have invalidated the randomization. Baseline demographic and clinical characteristics of the total sample of 184 patients in the study are shown in table 2. With reference to the psychological variables (type $\mathrm{A}$ behavior, vital exhaustion, depression), statistically significant differences between patients in the intervention group and control group were found on vital exhaustion, and a trend for significance was found for BDI depression. On average, patients randomized to the intervention group had higher scores on vital exhaustion $(p=$ $.05)$ and BDI depression $(\phi=.07)$ than patients in the control group (table 2). 


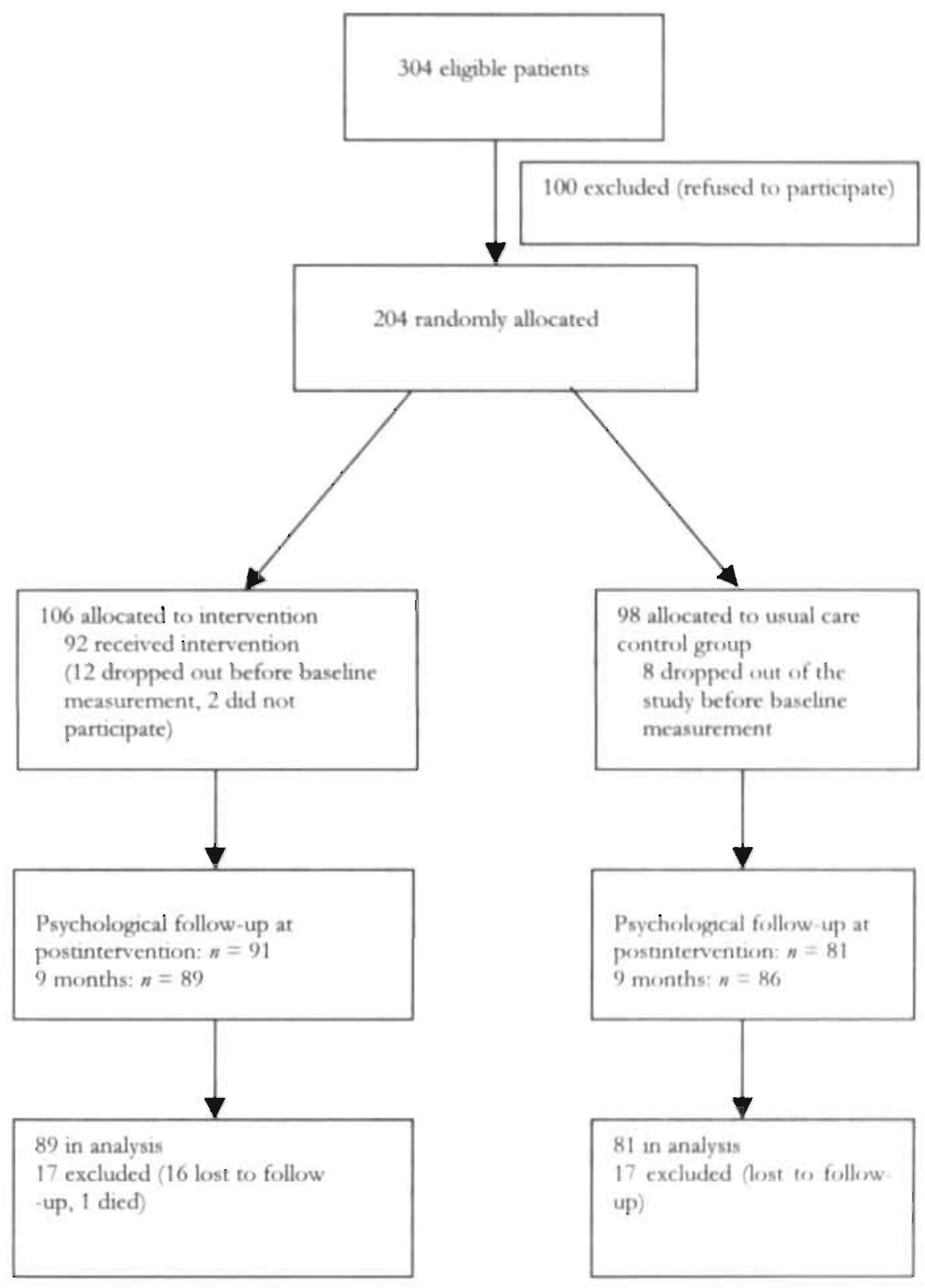

Figure 1. Flowchart of patients included in the study 
Table 2. Baseline characteristics of patients in control group $(n=94)$ and intervention group $(n=90)$

\begin{tabular}{|c|c|c|}
\hline Characteristic & $\begin{array}{r}\text { Intervention } \\
(\%)\end{array}$ & $\begin{array}{r}\text { Control } \\
(\%)\end{array}$ \\
\hline Male & 86.2 & 86.7 \\
\hline \multicolumn{3}{|l|}{ Clinical history } \\
\hline Previous myocardial infarction & 24.5 & 22.2 \\
\hline Previous CABG; & 4.3 & 2.2 \\
\hline Previous PTCA & 11.7 & 8.9 \\
\hline \multicolumn{3}{|l|}{ Reason for admission } \\
\hline Myocardal infarction & 73.4 & 66.7 \\
\hline Myocardial infarction + CABG & 7.4 & 7.8 \\
\hline $\mathrm{CABG}$ & 19.1 & 25.6 \\
\hline \multicolumn{3}{|l|}{ Medication at entry in the study } \\
\hline Beta-blockers $(2)^{\circ}$ & 86.0 & 82.0 \\
\hline Nitrates (2) & 37.6 & 29.2 \\
\hline Platelet inhibitor drugs or anticoagulants (2) & 89.2 & 87.6 \\
\hline \multicolumn{3}{|l|}{ Clinical status at entry in the study } \\
\hline Angina pectoris (3) & 28.3 & 16.9 \\
\hline Dyspnea (3) & 35.5 & 29.5 \\
\hline Mean age, years $[S D]$ & $55.6[8.0]$ & $55.2 \cdot[9.7]$ \\
\hline Eichocardiography at discharge: LVEF $|S D|(58)$ & $53.3[9.0]$ & $51.6[11.4]$ \\
\hline \multicolumn{3}{|l|}{ Psychological vanables } \\
\hline Type $A$ behavior (total) [SD! & $220.1|54.7|$ & $210.5[62.4]$ \\
\hline Vital exhauston $|S D|(1)^{*}$ & $6.2[4.9]$ & $4.8[4.5]$ \\
\hline Depression [SD] (BDI) (12) & $8.4[6.8]$ & $6.7|5.2|$ \\
\hline${ }_{0}^{\circ}$ Major Depression (SCID) (19) & 14.9 & 9.0 \\
\hline $\begin{array}{l}\text { Notf. Numbers in parentheses are numbers } \\
\text { vailable.C.ABG }=\text { Coronary Artery Bypass. Grat } \\
\text { oronary Angioplasty; L.VEF }=\text { Left Ventricular } \\
\text { nventury. Range of scores for each measuremen } \\
\text { 23; Depression (BDI) } 0-63 \text {. }\end{array}$ & $\begin{array}{l}\text { ients for wh } \\
A=\text { Percuta } \\
\text { Fraction; BDI } \\
\text { behavior 2-3 }\end{array}$ & $\begin{array}{l}\text { data are not } \\
\text { s: Transluminal } \\
\text { eck Depression } \\
\text { ital exhaustion }\end{array}$ \\
\hline
\end{tabular}

\section{Type A behavior}

Scores on type A behavior and its components at baseline and follow-up are reported in table 3. For two of the three different components of type $\mathrm{A}$ behavior; the intervention effect was significant. In the intervention group, the reduction of the cognitive hostility scores was 9.0 points larger than in the control group at postintervention (ANCOVA, 95\% CI - $15.1 /-2.8, F(1,164)=8.24, p=.01)$, and 7.7 points at 9 -month follow-up $(95 \% \mathrm{CI}:-14.5 /-0.9, F(1,16.4)=4.97, p=.03) .^{2}$ The reduction in time urgency scores was 11.5 points larger in the intervention group than in the control group at postintervention $(95 \% \mathrm{CI}:-19.8 /-3.2, F$ $(1,164)=7.49, p=.01)$ and 9.9 points larger at 9 -month follow-up $\left(95^{\circ} \circ \mathrm{CI}:-18.0\right.$ $/-1.9, F(1,164)=5.94, p=.02)$. The reduction in insecurity scores was 0.8 points

\footnotetext{
2 Reported differences between control group and intervention group in this and subsequent sections of chapter 2 are adjusted for baseline differences and aforementioned covarates (ANCOND).
} 
larger at postintervention, and at 9-month follow-up 1.8 points larger in the intervention group than in the control group, a difference that was not significant at postintervention $(95 \% \mathrm{CI}:-6.5 / 5.0, F(1,164)=0.07, p=.79)$ or at 9-month follow-up $(95 \% \mathrm{CI}:-7.5 / 4.0, F(1,164)=0.37, p=.54)$.

In a random sample of 50 patients, psychomotor characteristics of type $A$ behavior hardly changed from baseline to 9-month follow-up. The intervention group did not differ from the control group regarding change of psychomotor hostility and psychomotor time urgency scores, which was 0.6 points larger in the intervention group (hostility, 95\% CI: $-6.1 / 5.0, F(1,44)=0.04, p=.84 ;$ time urgency, $95 \%$ CI: $-8.4 / 7.3, F(1,44)=0.02, p=.88)$.

ANCOVA was performed with the 50 patients whose psychomotor type A characteristics were known, to compute the impact of the intervention on overall type A behavior (psychomotor plus cognitive type A behavior; not in table). The reduction in overall type $\mathrm{A}$ behavior at 9-month follow-up was 56.9 points larger in the intervention group than in the control group $(95 \% \mathrm{CI}:-92.0 /-21.7, F(1,44)=$ $10.63, p=.00)$. Figure 2 shows the reduction in overall type $A$ behavior at followup for intervention and control patients $(n=50)$. The reduction from baseline in type A behavior is expressed in overall SD at baseline.

Table 3. Type A behavior and its components - mean scores at baseline, at postintervention and at 9-month follow-up in the intervention and control group

\begin{tabular}{|c|c|c|c|c|c|c|}
\hline \multirow[t]{2}{*}{ Measure } & \multicolumn{3}{|c|}{ Intervention } & \multicolumn{3}{|c|}{ Control } \\
\hline & $M$ & $S D$ & $n$ & M & $S D$ & $n$ \\
\hline \multicolumn{7}{|c|}{$\begin{array}{l}\text { Type A behavior / cognitive: } \\
\text { Hostility }\end{array}$} \\
\hline Bascline & 74.1 & 20.8 & 89 & 71.2 & 238 & 81 \\
\hline Post & 50,3 & 25.2 & & 57.2 & 25.5 & \\
\hline 9 months & 53.6 & 25.3 & & 58.9 & 29.5 & \\
\hline \multicolumn{7}{|l|}{ Time Urgency } \\
\hline Bascline & $100 ! 1$ & 25.5 & 89 & 98.3 & 290 & 81 \\
\hline Post & 62.6 & 29.5 & & 72.8 & 32.3 & \\
\hline 9 months & 66.5 & 29.6 & & 75.0 & 32.1 & \\
\hline \multicolumn{7}{|l|}{ Insecurity } \\
\hline Baseline & 45.4 & 21.1 & 89 & 434 & 22.5 & 81 \\
\hline Post & 24.4 & 20.0 & & 24.1 & 21.9 & \\
\hline 9 months & 25,8 & 20.6 & & 26.3 & 22.6 & \\
\hline \multicolumn{7}{|c|}{$\begin{array}{l}\text { Type A behavior / psychomotor: } \\
\text { Hostility }\end{array}$} \\
\hline Baseline & 32.3 & 18.0 & 22 & 37.0 & 136 & 28 \\
\hline 9 months & $31: 6$ & 14.6 & & 35.0 & 11.6. & \\
\hline \multicolumn{7}{|l|}{ Time Urgency } \\
\hline Bascline & 42.1 & 15.2 & 22 & 50.0 & 14.3 & 28 \\
\hline 9 months & 42.1 & 13.2 & & 46.6 & 15.5 & \\
\hline
\end{tabular}

Note. Range of scores for each measurement: Cognitive: Hostility 2-145; Time Urgency 0-160, Insecurity 0-80. Psychomotor: Hostihty 0-110, Time Urgency 0-110. 


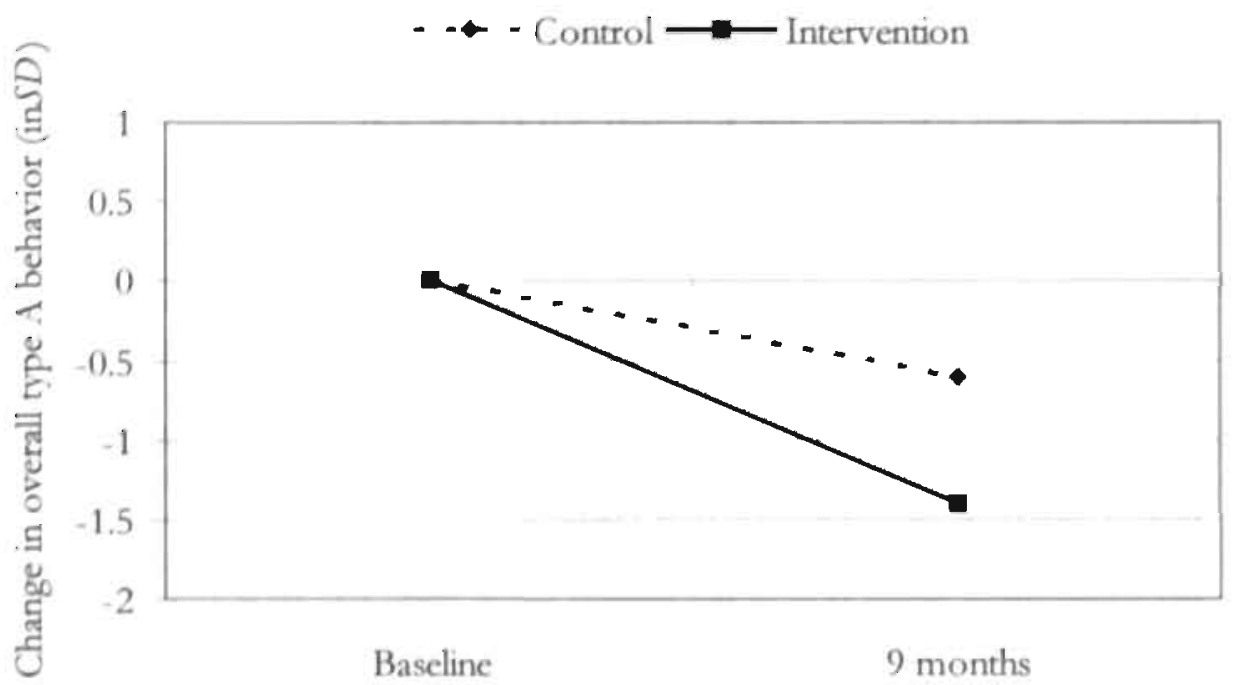

Figure 2. Changes in overall type $A$ behavior in SD at 9-month follow-up, for the control group and the intervention group. ${ }^{2}$

\section{Vital exhaustion}

Table 4 shows the scores on vital exhaustion and depression. Due to missing data there may be small differences in the number of subjects for the different outcome measures.

The intervention group showed a reduction in vital exhaustion at post intervention and 9-month follow-up, whereas the control group only showed a reduction at postintervention. No statistically significant differences were found between the scores of patients in the intervention and control group at postintervention; the reduction was 0.1 points larger in the control group (ANCOVA, 95\% CI: $-1.1 /$ $1.4, F(1,163)=0.05, p=0.82)$ and 1.0 points larger in the intervention group at 9month follow-up $(95 \% \mathrm{CI}:-2.5 / 0.5, F(1,163)=1.64, p=0.20)$.

Although no significant intervention effects were reported in the total study population on vital exhaustion, a significant $(p=.01)$ interaction of Group Allocation (control/intervention) by Baseline Score in the ANCOVAs showed that the effect of the intervention on vital exhaustion depended upon baseline scores. A more favorable effect of the intervention on vital exhaustion was found in patients with relatively high baseline scores, whereas no effects were reached in patients who scored low at baseline. This is illustrated in figure 3 .

Moreover, a sub-analysis with patients who scored 8 or higher on the interview for vital exhaustion showed that for this exhausted group of patients, the intervention group showed a reduction in vital exhaustion which was 4.7 points larger than in

2 Figure 2 - the difference between control group and intervention group in decrease of type $\mathrm{A}$ behavior from baseline to follow-up: $p=.002$. 
the control group after 9 months $(95 \% \mathrm{CI}:-8.7 /-0.6, F(1,37)=5,42, p=, 03)$. Whereas the vital exhaustion scores in the intervention group dropped from 11.5 [3.6] at baseline to 6.5 [6.5] at 9-month follow-up, the scores in the control group dropped from $12.0[3.4]$ at baseline to $11.5[6.0]$ at follow-up.

Table 4. Mean scores on psychological outcome measures at baseline, at postintervention and at 9-month follow-up in the intervention and control group

\begin{tabular}{ccccccc}
\hline Measure & \multicolumn{3}{c}{ Intervention } & & Control & \\
& $M$ & $S D$ & $n$ & $M$ & $S D$ & $n$ \\
\hline Vital Exhaustion & & & & & & \\
Baseline & 5.9 & 4.7 & 89 & 4.7 & 4.2 & 80 \\
Post & 5.5 & 5.3 & & 4.4 & 4.8 & \\
9 months & 4.6 & 5.7 & & 4.7 & 5.5 & \\
Depression (BDI) & 8.5 & 6.9 & 83 & 6.4 & 4.6 & 75 \\
Baseline & 7.7 & 6.0 & & 5.8 & 4.9 & \\
Post & 6.9 & 4.8 & & 5.8 & 5.1 & \\
9 months & & & & & & \\
\hline
\end{tabular}

Note. BDI = Beck Depression Inventory. Range of scores for each measurement: Vital exhaustion 0-23; Depression (BDI) 0-63.

\section{Control Intervention}

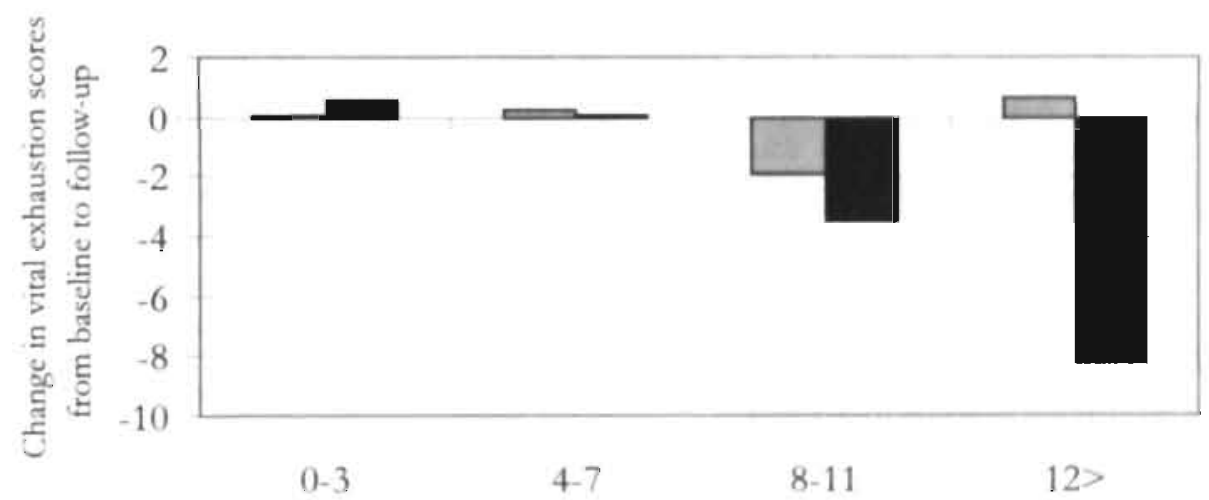

Baseline vital exhaustion scores

Figure 3. Change in vital exhaustion scores from baseline to 9-month follow-up for different baseline scores of vital exhaustion. 


\section{Depression}

Differences between the intervention and control group in the scores of depression (BDI) at postintervention and at 9-month follow-up were not significant; the reduction was 0.5 points larger in the control group at postintervention (ANCOVA, 95\% CI: $-0.8 / 1.7, F(1,152)=0.50, p=.48)$ and 0.1 points at 9-month follow-up $(95 \% \mathrm{CI}:-1.3 / 1.4, F(1,152)=0.01, p=.92) . \mathrm{A}$ nearly significant $(p=.06)$ interaction in the ANCOVAs showed that the favorable effect of the intervention on depression at 9-month follow-up was larger in patients with unfavorable baseline scores of depression than in patients who scored more favorably. A sub-analysis with patients who scored high on BDI depression at baseline, however, revealed no significant intervention effects, since the reduction was only 0.9 points larger in the intervention group than in the control group $(95 \% \mathrm{CI}:-3.8 / 2.1, F(1,42)=0.34, p=.56)$.

The percentage of depressed patients as measured by the SCID interview increased in the intervention group from $15 \%$ at baseline to $17 \%$ at postintervention and $21 \%$ at 9 -month follow-up; in the control group $9 \%$ were depressed at baseline, $5 \%$ at postintervention and $18 \%$ at 9 -month follow-up. Effects on depression as measured by this interview were assessed by means of logistic regression analyses. At postintervention, the odds ratio (OR) for depression as measured by the SCID interview was 3.68 in favor of the control group ( $95 \%$ CI: $1.10 / 12.37, p=.04$ ). The difference between the intervention group and the control group disappeared at 9-month follow-up (OR $=1.01,95 \% \mathrm{Cl}: 0.44 / 2.31, p=.99)$.

\section{Morbidity and mortality}

The intervention group did not differ from the control group in number of recurrent events at any of the follow-up measurements. All patients survived during the 9-month follow-up period, apart from one patient in the intervention group. The cause of death was non-cardiac (cancer). A total of 17 patients had experienced one or more recurrent cardiovascular events during the nine months of follow-up; 9 in the intervention group and 8 in the control group. Ten patients had had a CABG operation (6 in the intervention group and 4 in the control group), and 8 patients underwent a PTCA ( 3 in the intervention group and 5 in the control group) during the follow-up period. None of the patients experienced a (recurrent) myocardial infarction.

\section{DISCUSSION}

The results of this study show that type $A$ behavior can be reduced in coronary patients through a relatively short intervention program aimed at behavior change and risk reduction. In addition, patients who were exhausted at baseline showed less vital exhaustion after 9 months compared to control patients. The reductions that were found in type $\mathrm{A}$ behavior and vital exhaustion may have favorable consequences for the prognosis of coronary patients, since these factors have been 
associated with increased risk of a coronary incident (Brackett \& Powell, 1988; Kop et al., 1994). Moreover, it seems that the reductions can be achieved in a relatively short period of time.

Caution is warranted, however, since the favorable reductions we found in type A behavior were not reflected in the psychomotor characteristics of the behavior pattern. Therefore, the possibility that intervention patients gave socially desirable answers to the questions concerning type $\mathrm{A}$ behavior cannot be completely ruled out.

Type A behavior was not only reduced in the intervention group, but in the control patients as well. In our study, several factors may account for this decline. First, patients after AMI or after a $\mathrm{CABG}$ probably try to change their behavior spontaneously to reduce the risk of a recurrent event. The interview to assess type A behavior at baseline - which was repeated at follow-up - may have made patients more aware of their own behavior, which subsequently stimulated behavior change. After each interview, the interviewer gave a short summary of the major contents of each part of the interview, including the results of the Structured Interview assessment. Second, the reduction in the control group might partly be the result of contamination of treatment. Patients of both groups were able to communicate with each other during the collective weekly physical therapy sessions that formed a part of their usual care treatment, so information about the program could have been exchanged.

Could the favorable effects of the intervention on type $A$ behavior and vital exhaustion be explained by regression to the mean, especially since the effects were found in the high-risk group? In the total study population, the intervention group had higher baseline scores than the control group, a difference that can only be explained by coincidence, since randomization was performed with allocation concealment, and by a person not further involved in the study. Because of the higher baseline exhaustion scores, the intervention group can be expected to show a larger reduction in those scores than the control group. In the subgroup-analysis of patients with high levels of exhaustion, however, the control group and the intervention group did not differ in baseline scores. Thus, the fact that vital exhaustion in the intervention group dropped tor a lower level than in the control group cannot be explained by regression to the mean.

No favorable effects were found on depression. Percentage of patients with major depression as classified by the SCID even declined in the control group at postintervention, but not in the intervention group. This negative finding dissipated at 9-month follow-up. Moreover, in accordance with previous studies, among the dropouts a higher percentage of patients were depressed than among the non-dropouts (Cooper, Lloyd, Weinman, \& Jackson, 1999; Lane, Carroll, Ring, Beevers, \& Lip, 2001). Since depression constitutes a risk for future cardiac events, this is an alarming observation for which a solution has not yet been found (Carney, Freedland, Veith, \& Jaffe, 1999; Lesperance \& Frasure-Smith, 2000; Wulsin, Vaillant, \& Wells, 1999).

Although no significant effects were found in the total study group for vital exhaustion and depression as measured with the BDI, it should be noted that the 
intervention focused more directly at modifying type $\mathrm{A}$ behavior than at altering depression and vital exhaustion, which were addressed more indirectly. Specific aftention for vital exhaustion and depression might have increased favorable intervention effects.

\section{Comparison with relevant studies}

In a review on risk factor modification through nonpharmacological interventions in coronary patients (Sebregts et al., 2000), three major randomized clinical trials were found evaluating an intervention aimed at type $\mathrm{A}$ behavior modification (Burell, 1996; Burell et al., 1994; Friedman et al., 1986). All three studies reported significant reductions of type A behavior after a lengthy, time-consuming intervention. One of these studies reported a significant reduction in BDI scores for the treatment group compared with the control group as well (Burell, 1996). The considerable advantage of our program over previous trials is that the patients were offered a relatively short intervention, which is easily applicable in general health care settings. In our study, overall type A behavior decreased after 9 months by $1.4 S D$ in the intervention group, whereas the control group showed a decrease of 0.6 SD. The Recurrent Coronary Prevention Project (RCPP) showed comparable reductions in Videotaped Structured Interview scores of type A behavior after comprehensive type A behavioral counseling during 4.5 years (Friedman et al, 1986).

In a meta-analysis published by Dusseldorp et al (Dusseldorp et al., 1999), the effects of psychoeducational health education and stress management programs for coronary heart patients were examined. In this meta-analysis, no effects of psychoeducational programs were found in regard to depression. Several large studies were included in this meta-analysis (Frasure-Smith et al., 1997; Jones \& West, 1996). The results of the study presented in this paper do not differ from the majority of these previous studies. In order to reduce depression in patients with coronary artery disease, we may need to adapt the contents of our interventions.

\section{Limitations of the study}

The sub-analyses with patients with high baseline scores were not preplanned, but were conducted since baseline scores on psychological variables in our study group were generally low. The total study population scored 5.3 on the MIVE. Although this is higher than a mean score of 3.6 observed in healthy males, it is considerably lower than a score of 8 or higher, at which point persons are said to be exhausted (Meesters \& Appels, 1996b). The mean BDI score for depression in our study was 7.5, while persons scoring 10 or higher are considered depressed. So, in these patients, little improvement could be expected, and positive intervention effects might have been found if patients had been more distressed at baseline. This is indeed suggested by the positive interactions that were found with intervention effects and baseline measurements. Higher scores of vital exhaustion and depression were associated with larger favorable effects of the intervention. Future intervention studies should focus on more distressed study populations. 
This study was not conducted around one primary outcome measure, but we used multiple outcomes. Multiplicity of outcomes increases the risk of some falsepositives. In a study such as this, more than one measure of outcome is relevant, however, which is illustrated by the fact that most studies on cardiac rehabilitation programs use multiple outcome measures. Moreover, if we correct the positive results for type A behavior for multiplicity of outcomes, significant results are still found for the favorable reduction in overall type A behavior after 9 months, and for the favorable reductions in cognitive hostility scores at postintervention as well as for the reduction in cognitive time-urgency scores at postintervention and at 9 month follow-up.

Due to the design of the study it is impossible to indicate which elements or components of the intervention attributed to the positive results. We could not control for nonspecific aspects of the intervention such as attention and the mutual support given by the participants. We merely wanted to study whether a short intervention can be effective in reducing detrimental psychological factors in CAD patients. Consequently, the study was designed with two arms only: intervention and usual care. No separate arm, for example, was formed by an intervention without attention to type $\mathrm{A}$ behavior. Therefore we cannot say that especially this part of the intervention resulted in the reduction of type $\mathrm{A}$ behavior. Another weakness is formed by the absence of a thorough registration of exercise habits. It is possible that changes in exercise have contributed to the beneficial effects of the study. However, we consider that to be an unlikely explanation because all patients were stimulated to exercise as part of the standard physical rehabilitation program all patients. received.

Despite these limitations, the results of this intervention study indicate relatively large and persistent reductions in type $A$ behavior, although this is not reflected in the patients' psychomotor characteristics of type A behavior. Although no main effects of the intervention are seen in scores of vital exhaustion, the results indicate reductions in vital exhaustion after the intervention in patients with high baseline exhaustion scores, thus, those who need it. No main intervention effects are found in BDI depression scores. The higher reduction in percentage of patients with major depression that was found at postintervention in the control group compared to the intervention group, however, should make us cautious of advising the intervention to the general population of AMI and CABG patients under the the age of 70 . 
Chapter 2 
APPENDIX TO CHAPTER 2: SECONDARY PSYCHOLOGICAL OUTCOME MEASURES 


\section{Introduction}

The aforementioned results show the primary psychological outcome measures of this study, i.e. type A behavior, vital exhaustion and depression. Quality of life and anxiety served as secondary outcome measures, and the results concerning these outcomes are reported below (table A). The results will briefly be described in the general discussion of this thesis.

In addition, post-hoc analyses will be presented in which differential effects of the intervention were studied for men and women. For this purpose, an interaction variable Group Allocation by Gender was included in the aforementioned ANCOVAs for studying intervention effects on psychological variables.

\section{Secondary psychological outcome measures}

Quality of life was determined by a Dutch translation of the Multidimensional Index of Life Quality (MILQ), which is a 35-item instrument for measuring quality of life in patients with cardiovascular disease (Avis et al., 1996). Each item is scored on a 7-point scale reflecting the respondent's degree of satisfaction with the item, ranging from 1 (= very dissatisfied) to 7 (= very satisfied). The total score was computed by taking the unweighted sum of all scores.

Anxiety was measured by administering a Dutch version $(\mathrm{ZBV})$ of the Spielberger State-Trait Anxiety Inventory (STAI) (Spielberger, Gorsuch, \& Lushene, 1970; Van der Ploeg, Defares, \& Spieiberger, 1980). Both the State and Trait scales of the STAI contain 20 items. Each item is scored on a. 4-point scale, ranging from 1 (= not at all) to 4 (= very much).

Table A. Results of ANCOVA comparing the intervention and control group on postintervention and at 9-month follow-up scores of quality of life and anxiety, in. which baseline scores are used as a covariate

\begin{tabular}{cccccccc}
\hline Measure & \multicolumn{3}{c}{ Intervention } & \multicolumn{5}{c}{ Control } \\
& $M$ & $S D$ & $n$ & $M$ & $S D$ & $n$ & $F$ \\
\hline & & & & & & & \\
Quality of Life & & & & & & & \\
Baseline & 178.0 & 32.7 & 83 & 183.7 & 26.8 & 76 & \\
Post & 189.5 & 29.5 & & 190.6 & 25.4 & & 2.13 \\
9 months & 190.0 & 28.1 & & 192.0 & 27.0 & & 0.39 \\
State Anxiety & & & & & & & \\
Baseline & 39.0 & 11.8 & 80 & 38.0 & 9.2 & 75 & \\
Post & 38.8 & 11.9 & & 35.4 & 10.6 & & 3.829 \\
9 months & 38.0 & 10.9 & & 35.6 & 9.6 & & 2.12 \\
Trait Anxiety & & & & & & & \\
Baseline & 38.0 & 11.5 & 80 & 36.8 & 8.6 & 76 & \\
Post & 38.3 & 11.5 & & 36.1 & 10.0 & & 0.81 \\
9 months & 36.6 & 10.7 & & 35.5 & 9.5 & & 0.04 \\
\hline
\end{tabular}

Note. Range of scores for each measurement: Quality of Life 35-245; State and Trait Anxiety 20-80. "Covariates in these analyses were baseline score, gender, age.

"AMl (+CABG) vs. CABG only". "p<.05. $p<10$ 


\section{Gender differences in intervention effects}

Previous publications raised the suggestion that interventions may have a different effect on men and women (Brezinka and Kittel, 1995; Frasure-Smith et al., 1997; Linden, 2000; Linden et al., 1996; McGee and Horgan, 1992). In our study, the interaction variable Group Allocation by Gender was nearly significant or significant in the analyses of vital exhaustion $(p<.10)$, quality of life $(p<.05)$, state anxiety $(p<.05)$ and trait anxiety $(p<.10)$, suggesting more favorable intervention effects for men than for women. There were not enough women in our study to conduct separate analyses for women. The number of men was large enough to conduct separate analyses. Although sub-analyses for men did not yield significant intervention effects on the reduction of anxiety, we found (with a trend for significance) that the intervention was effective in reducing vital exhaustion. At 9 month follow-up, men in the intervention group showed a 1.5 larger decline in vital exhaustion (baseline 5.82, 9 months $4.18, n=77$ ) compared to control patients (baseline 4.45, 9 months 4.69, $n=71$ ), ANCOVA, 95\% CI: $-3.0 / 0.1, F$ $(1,143)=3.26, p=.07$. Moreover, the increase in quality of life was 5.7 points larger in men in the intervention group compared to controls at postintervention, $95 \% \mathrm{CI}: 0.3 / 11.2, F(1,133)=4.39, p=.04$, and 5.2 points larger at 9-month follow-up, $95 \%$ CI: $-0.9 / 11.3, F(1,133)=2.83, p=.09$ (Intervention: baseline 177.6, post 189.9, 9 months 191.4, $n=71$; Control: baseline 184.0, post 190.0, 9 months 191.3, $n=67$ ). 

C.HAPTER 3

CHOLESTEROL CHANGES IN CORONARY PATIENTS AFTER A SHORT BEHAVIOR MODIFICATION PROGRAM

\section{Publisbed as:}

Sebregts, E.H., Falger, P.R., Bär, F.W., Kester, A.D., and Appels, A. (2003) Cholesterol changes in coronary patients after a short behavior modification program. International Journal of Behavioral Medicine, 10, 315-330. 


\section{ABSTRACT}

Serum cholesterol changes after an 8-week behavior modification program for patients with coronary artery disease (CAD) were studied in a randomized controlled clinical trial. Acute myocardial infarction (AMI) or coronary artery bypass grafting (CABG) patients were randomly assigned to the intervention $(n=$ $94)$ or to usual care $(n=90)$. After 9 'months' follow-up the intervention was effective in reducing total cholesterol and LDL cholesterol levels, particularly in patients with high baseline lipid levels. After correcting for changes in dose of statins during follow-up, effects were weakened, but for patients with high baseline cholesterol levels favorable effects remained. In these patients, the intervention. group showed a decline of total cholesterol and LDL cholesterol levels of $20 \%$ and $29 \%$, respectively, compared to a $12 \%$ and $19 \%$ reduction in the control group $(p$ $<.01)$. These effects could not be explained by changes in dietary fat consumption. An unexpected finding was a lower increase in HDL cholesterol in the intervention group than in the control group. 


\section{INTRODUCTION}

In clinical practice, the standard procedure to reduce the risk exerted by an elevated cholesterol level in coronary artery disease (CAD) patients is through pharmacological treatment, i.e., cholesterol-lowering agents (usually statins) and a cholesterol-lowering diet. Since the statins are already quite effective in reducing cholesterol levels (Sacks et al., 1996; Scandinavian Simvastatin Survival Study Group, 1994), the question arises whether behavior modification interventions can make an additional contribution to this reduction.

In a recent review on the effects of nonpharmacological interventions for CAD patients, it was concluded that interventions aimed at a healthy lifestyle reduce elevated serum total and LDL cholesterol concentrations in CAD patients (Sebregts et al., 2000). Some of the studies reported in this review even showed a reduction of coronary atherosclerosis after successful modification of patients' lifestyles. (Niebauer et al., 1997; Ornish et al., 1990; Watts et al., 1992). However, the use of statins nowadays is more widespread than during the time these studies were conducted. Moreover, such favorable effects were found in trials evaluating intensive interventions with a duration of at least one year and was probably reached only in a selective group of highly motivated CAD patients (Nicbauer et al., 1997; Ornish et al., 1990).

If interventions need to be this comprehensive to successfully alter elevated serum cholesterol levels and other risk factors, probably only a minority of eligible CAD patients will be reached. Moreover, such interventions are too expensive and too time-consuming to implement in general health care. Applicability of behavioral interventions in general health care would be improved if interventions were short - but nonetheless as effective as the comprehensive ones.

In the present study it was intended to alter serum cholesterol levels in acute myocardial infarction (AMI) or Coronary Artery Bypass Grafting (CABG) patients through a relatively short intervention, which is directed at the improvement of a healthy lifestyle - dietary habits in particular - and reduction of psychological stress." This paper tackles the following questions: (1), Does this intervention lead to reduced serum cholesterol levels? (2) If so, can the reduced cholesterol levels be explained by (a) altered medication regimen, or by (b) reduced dietary fat consumption?

\section{METHODS}

\section{Participants}

All patients admitted to the University Hospital Maastricht during the period of February 1996 until November 1997 were identified as eligible for the study by a team of physiotherapists. working at the hospital's physiotherapy department.

Psychological effects of the intervention will be published elsewhere. 
These patients were referred to them by cardiologists and cardiac surgeons for physical training after AMI or CABG. Patients included were $<70$ years of age; who were admitted to the University Hospital Maastricht with the confirmed diagnosis of $\mathrm{AMI}, \mathrm{CABG}$, or both; and who were able to participate in the regular physiotherapy exercise-program after discharge from the University Hospital Maastricht. The number of parients that was included in this study was based on feasibility within the time limits of the study. Patients were excluded if they were non-Dutch-speaking, illiterate, or were currently suffering from any psychiatric disorder that would severely disturb participation in the intervention. During this 21 -month period, signed informed consent was given by 204 patients $(67 \%$ of all eligible patients).

\section{Procedure}

After informed consent was given, patients were randomly assigned to either the intervention $(n=106)$ or to the control group $(n=98)$. Both groups received usual medical care. Patients that were assigned to the intervention group enrolled into a multiple risk modification program for secondary prevention of CAD. This intervention started within 3-8 weeks after discharge.

Questionnaires concerning dietary habits and interviews concerning smoking for the intervention group were assessed (1) at baseline, before the beginning of the intervention, and (2) 9 months after termination of the intervention (approximately one year after admission). Measures for control patients were collected at comparable moments in time. The interviewers remained unaware of patient group assignment.

\section{Usual care}

Both the control group and the intervention group received the same usual medical care, consisting of regular check-ups by a cardiologist who was blinded to patient group allocation, and postdischarge exercise training sessions. In the Netherlands, exercise training is part of the usual care for patients with AMI or CABG. Patients received standardized exercise training three times a week during 6 weeks.

During the regular medical check-ups, standard usual care was offered, comparable to the care patients received who did not participate in this study, This meant that the cardiologist systematically checked the clinical history, performed physical examination, did a 12-lead ECG recording, and, if appropriate, performed blood tests. The cholesterol level of each patient was followed until his or her cholesterol reached satisfactory levels. The cardiologist provided concise information on risk modification like unfavorable cholesterol levels and the favorable effects on prognosis of lowering cholesterol. In case of elevated values at baseline, patients were advised to eat a low-fat diet, and cholesterol-lowering drugs were given, after which serum cholesterol was measured and amount of drugs was increased until the results were satisfactory. The following were considered high levels: elevated total cholesterol levels $>5.0 \mathrm{mmol} / \mathrm{L}(193 \mathrm{mg} / \mathrm{dl})$, elevated LDL cholesterol $>3.0$ 
$\mathrm{mmol} / \mathrm{L}(116 \mathrm{mg} / \mathrm{dl}$ ) (as these were the cholesterol levels above which it was advised to intervene), reduced $\mathrm{HDL}$ cholesterol $<1.0 \mathrm{mmol} / \mathrm{L}(39 \mathrm{mg} / \mathrm{dl})$, and elevated level of triglycerides $>2.0 \mathrm{mmol} / \mathrm{L}(177 \mathrm{mg} / \mathrm{dl})$. In the patients already treated with cholesterol-lowering drugs, further titration of this medication was performed if needed. For each AMI patient exercise-tests were performed according to the Bruce Protocol during hospitalization, and one year after discharge. The CABG patients only had an exercise test one year after discharge.

\section{Intervention}

In addition to the aforementioned usual care, patients allocated to the intervention group were offered a combined stress-management and health education program during eight weekly 2.5-hr sessions. The contents of the intervention were primarily based on the methods of three major long-term clinical trials, the Recurrent Coronary Prevention Project (Bracke \& Thoresen, 1996), Project New Life (Burell, 1996), and the Lifestyle Heart Trial (Omish et al., 1990), adapted to a format that would be feasible within the context of a short intervention program. During the eight weekly sessions, 6 to 10 cardiac patients and their partners (whose participation was encouraged) were informed about CAD risk factors and risk factor modification by a multidisciplinary team. The first hour-and-a-half consisted of interactive didactic presentations of these issues, including advice, exercises to actively modify each participant's CAD risk factor profile, or both. Patients were given advice to eat a low-fat diet, how to do so, and the health benefits of radical low-fat dietary changes were pointed out to them. Special attention was paid to the favorable effects of a vegetarian diet, and information was given on cholesterol, e.g., the benefits of a low rotal and I.DI. scrum cholesterol. In the remainder of each session participants were encouraged to report their experiences with the issues at hand. Each session was concluded with breathing and relaxation exercises based on the work of Van Dixhoorn (Van Dixhoorn, 1997). The purpose of these exercises was primarily to increase the patients' sense of relaxation and body awareness. At the end of most sessions, patients were given homework assignments, as well as written information, with reference to the specific topics mentioned earlier.

\section{Clinical dạta}

Clinical data were collected by a cardiologist who was blinded to the parients' group allocation. Serum cholesterol (total, LDI, HDL, triglycerides) used for the study was measured in a fasting state, at least at bascline and at 9-month followup. Height of the patients was measured during the first outpatient visit, whereas weight was measured during all visits. From height and weight measures, Body Mass Index (BMI) was calculated.

Additional clinical data, used for baseline group comparisons, were collected through structured inventones, which were filled out by the same cardiologist. Among other things, this baseline inventory contained items concerning the patient's medical history (obtained from the medical files), angina pectoris, 
dyspnea, enzymatic infarct size during hospitalization, medication, and echocardiographic as well as angiographic data, if available. In addition, during follow-up cardiac complaints and cardiac or other events were recorded.

\section{Interviews and questionnaires}

Dictary fat consumption was measured through a self-report questionnaire, in which the respondent is asked about his or her food consumption during the last 4 weeks (Feunekes, Van Staveren, De Vries, Burema, \& Hautvast, 1993; Feunekes, Van Staveren, Graveland, De Vos, \& Burema, 1995; Wageningen University, 1993). From this questionnaire, consumption of fat (in grams/day) could be calculated by means of a computer program (Wageningen University, 1993). Selfreported smoking habits and alcohol consumption were measured through a structured interview on lifestyle (Falger, 1989).

\section{Data analysis}

\section{Initial anahyses}

First, does the intervention lead to reduced serum cholesterol levels? Analyses were performed according to the intention to treat principle, meaning that patients were analyzed in the original groups formed by randomization. All patients allocated to the intervention group were included in the analyses irrespective of their level of participation. Effects of the intervention on cholesterol level were assessed by means of analyses of covariance (ANCOVA), using the baseline cholesterol levels as a covariate to correct for differences in scores at baseline between the control and intervention group. In addition, for each of the outcome measures, the variables gender, age, cholesterol-lowering medication and antidiabetic medication at baseline, and "CABG vs. no CABG" were used as covariates in the analyses. One of the reasons this last variable was included was because cholesterol levels can be temporarily reduced as a consequence of a CABG operation itself.

After these main analyses, an interaction variable (Group Allocation $\mathrm{x}$ Baseline Cholesterol Level) was included in the ANCOVA to check whether intervention. effects were different for patients with high or low baseline cholesterol levels. A posteriori, we compared change in alcohol consumption, smoking rates and BMI from baseline to follow-up between control and intervention group, as these factors may be related to cholesterol level.

Second, can reduced cholesterol levels in the intervention group be explained by (a) altered medication regimen, or (b) reduced dietary fat consumption? (a) To check whether (possible) effects could be explained by altered medication regimen, changes of cholesterol-lowering medication from baseline to follow-up were studied for the intervention group and the control group. Changes of cholesterollowering medication from baseline to follow-up were then added as a covariate in the ANCOVA, to study whether this led to any changes in reported intervention effects. (b) To study whether changes in fat consumption were different in intervention and control group, an ANCOVA was performed in which baseline dietary fat consumption was used as a covariate. Other covariates were copied 
from the cholesterol analyses (with the exception of baseline cholesterol-lowering medication). An interaction variable was later added to the model to study whether results were different for patients with high or low baseline dietary fat consumption. In case of a significant interaction, separate analyses were performed for patients with high $(>80 \mathrm{~g}$ fat intake per day, based on guidelines for fat consumption carried out by Dutch nutritionists) and low ( $\leq 80 \mathrm{~g}$ fat intake per day) baseline dietary fat consumption. In order to study whether the reported changes in dietary fat consumption contribute to changes in serum cholesterol level, an ANCOVA was performed in which change in dose of statins from baseline to follow-up was used as a covariate.

\section{I ast obsenvation carried fonuard' analyses}

After these initial analyses, the aforementioned analyses were repeated using the method of 'the last observation carried forward'. Missing values at follow-up were replaced by the last observation, which was carried. forward. Missing data at baseline were replaced by the mean of all baseline values, provided that at least one follow-up measurement was available.

\section{RESULTS}

\section{Baseline characteristics and number of patients remaining in this study after 9 months}

In spite of considerable efforts to reduce missing data to a minimum, dropouts could not be avoided. Of all 204 patients included in the study, 20 dropped out, reducing the total number of patients in the study to 184 . Since they dropped out before baseline measurement - the time at which parients received their group allocation, these patients were not aware of group allocation. Baseline demographic and clinical characteristics of the total sample of 184 patients in the study are shown in table 1. No major differences were found between the intervention and control group.

\section{Initial analyses}

\section{Effect of the intenvention on serum cholesterol levels}

Table 2 shows changes in serum lipid levels during the intervention period. After 9 months, serum total cholesterol dropped by $8 \%$ in the intervention group, and by $2 \%$ in the control group. The difference in reduction of total serum cholesterol level between both groups reached statistical significance, $F(1,160)=5,45, p=$ .02. A stronger decline in L.DL cholesterol $(15 \%)$ was observed in the intervention group than in the control group $(10 \%)$, which was close to significance, $F(1,157)$ $=3.48, p=.06$. HDL cholesterol increased both in the intervention group $(11 \%)$ 
and in controls $(23 \%)$, with a significant difference between both groups in favor of the control group, $F(1,157)=9.90, p=.00$. Triglycerides showed a slight decrease in both groups, without any difference between the control group and the intervention group, $F(1,159)=0.22, p=.64$. The effects of cholesterol-lowering medication will be described shortly.

Table 1. Baseline characteristics of patients in intervention and control group

\begin{tabular}{|c|c|c|}
\hline Characteristic & $\begin{array}{r}\text { Intervention } \\
(n=94)\end{array}$ & $\begin{array}{r}\text { Control } \\
(n=90)\end{array}$ \\
\hline Male, $\%$ & 86.2 & 86.7 \\
\hline Mean age, yr $|S D|$ & $55.6|8.0|$ & $55.2[9.7]$ \\
\hline \multicolumn{3}{|l|}{ Clinical history } \\
\hline Previous myocardial infarction, $\%$ & 24.5 & 22.2 \\
\hline Previous CABG, \% & 4.3 & 2.2 \\
\hline Previous PTCA, $\%$ & 11.7 & 8.9 \\
\hline \multicolumn{3}{|l|}{ Reason for admission } \\
\hline Myocardial infarction, $\%$ & 73.4 & 66.7 \\
\hline Myocardial infarction + CABG; \% & 7.4 & 7.8 \\
\hline CABG, $\%$ & 19.1 & 25.6 \\
\hline \multicolumn{3}{|l|}{ Clinical status at entry in the study } \\
\hline Angina pectoris, $\%(3)^{*}$ & 28.3 & 16.9 \\
\hline Dyspnea, $\%(3)^{2}$ & 35.5 & 29.5 \\
\hline Infarct size: $A S A T$ maximum, U/1. [SD] (2)" & $174.3[138.2 \mid$ & $193.2[180.1]$ \\
\hline I.VEF, $\%|S D|(58)^{*}$ & $53.3[9.0]$ & $51.6|11.4|$ \\
\hline Fixercuse tolerance, munutes $[S D]^{\mathrm{t}}$ & $8.8[2.2]$ & $8.7\lfloor 2.3\rfloor$ \\
\hline \multicolumn{3}{|c|}{$\begin{array}{l}\text { Nofe. CABG = Coronary Artery Bypass Grafting, PTCA = Percutaneous Transluminal } \\
\text { Coronary Angioplasty; ASAT = Aspartate Aminotransferase; I.VEF = Left Ventricular } \\
\text { Fiection Fraction. } \\
\text { "Numbers in parentheses are numbers of patients for whom data were missing. } \\
\text { bixercise tolerance at entry in the study (walk test according to Bruce protocol) } \\
\text { was only avalable for } 90 \mathrm{AMI} \text { patients and not for CABG patients. }\end{array}$} \\
\hline
\end{tabular}


Table 2. Serum lipid levels in the intervention and control group at baseline and at 9-month follow-up"

\begin{tabular}{|c|c|c|c|c|c|c|}
\hline \multirow[t]{2}{*}{ Measure } & \multicolumn{3}{|c|}{ Intervention } & \multicolumn{3}{|c|}{ Control } \\
\hline & $M$ & $S D$ & $n$ & $M$ & $S D$ & $n$ \\
\hline \multicolumn{7}{|c|}{ Total Cholesterol (mmol/L) } \\
\hline Baseline & 5.17 & 1.22 & 84 & 5.09 & 1.03 & 84 \\
\hline 9 months & 4.74 & 0.78 & 84 & 4.97 & 0.81 & 84 \\
\hline \multicolumn{7}{|c|}{ LDL Cholesterol (mmol/L) } \\
\hline Baseline & 3.48 & 1.12 & 83 & 3.47 & 0.98 & 82 \\
\hline 9 months & 2.95 & 0.68 & 83 & 3.14 & 0.79 & 82 \\
\hline \multicolumn{7}{|c|}{ HDL Cholesterol (mmol/L) } \\
\hline Bascline & 0.95 & 0.30 & 83 & 0.94 & 0.27 & 82 \\
\hline 9 months & 1.05 & 0.30 & 83 & 1.16 & 0.32 & 82 \\
\hline \multicolumn{7}{|c|}{ Tngiycendes $(\mathrm{mmol} / \mathrm{L})$} \\
\hline Baseline & 1.91 & 1.25 & 84 & 1.68 & 0.71 & 83 \\
\hline 9 months & 1.77 & 1.00 & 84 & 1.57 & 0.84 & 83 \\
\hline
\end{tabular}

Note. "To convert cholesterol values from $\mathrm{mmol} / \mathrm{L}$ to $\mathrm{mg} / \mathrm{dl}$, multaply by 38.67. To convert triglyceride values from mmol/ $\mathrm{L}$. to $\mathrm{mg} / \mathrm{dll}$, mulaply by 88.57 .

\section{Patients with elevated cholesterol levels}

The interaction variable Group Allocation $\mathrm{x}$ Baseline Cholesterol Level reached significance for total cholesterol $(p=.02)$ and L.DL cholesterol $(p=.01)$; thus, effects of the intervention on total cholesterol and I.DI. cholesterol were significantly related to baseline lipid level. The effect of the intervention on reducing total serum cholesterol was present only in patients with relatively high baseline cholesterol. This is illustrated in figure 1, which shows the changes in cholesterol levels in the intervention and control group for patients with different cholesterol levels at baseline. In patients with elevated baseline total cholesterol levels (i.e., > $5.0 \mathrm{mmol} / \mathrm{L}$ ), total cholesterol dropped by $20 \%$ in the intervention group, whereas in the control group total cholesterol level fell by $12 \%, F(1,75)=$ $8.58, p=.00$.

In patients with elevated total cholesterol levels $(>5.0 \mathrm{mmol} / \mathrm{L}), \mathrm{LDL}$ cholesterol fell by $29 \%$ in the intervention group. The reduction was smaller in the control group, where patients showed a $19 \%$ decrease in LDL cholesterol, $F(1,75)=9.56$, $p=.00$. In patients with elevated cholesterol levels, no intervention effects were found for HDL cholesterol, $F(1,75)=1.64, p=.20$ and triglycerides, $F(1,75)=$ $0.09, p=.76$. 
Control Intervention

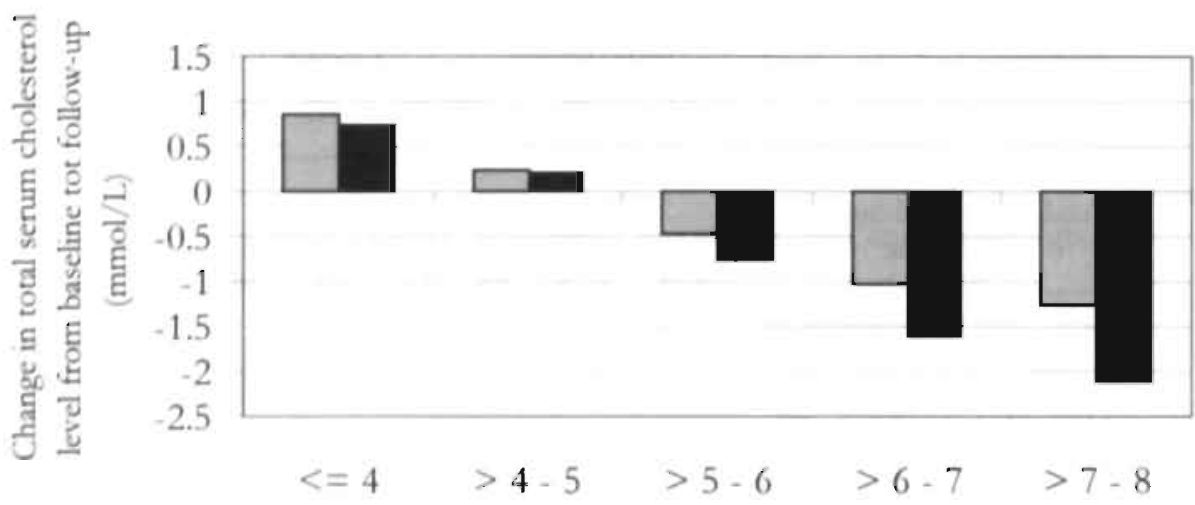

Baseline total serum cholesterol level $(\mathrm{mmol} / \mathrm{L})$

Figure 1. Change in total serum cholesterol level from baseline to 9-month followup in intervention and control group.

Alcohol consumption, smoking rates, and BMI could not account for any of the effects, as no difference was found between the intervention and the control group in change in alcohol consumption from baseline to follow-up $(n=164, p=.76)$, number of patients that had quit smoking (intervention group $21.3 \%$, control group $22.2 \%, n=170, p=.89$ ), and change in BMI (intervention group increase of $1 \%$, control group $2 \%, n=165, p=.67$ ).

\section{Altered medication regimen}

To check whether the difference in reduction of total serum cholesterol and LDL cholesterol between the intervention and control group could be explained by a difference in cholesterol-lowering medication regimen during follow-up, both groups were a posteriori compared with regard to change in prescribed dosage of cholesterol-lowering medication from baseline to follow-up. Cholesterol-lowering medication at baseline and at follow-up is described in table 3. At 9-month followup, $81 \%$ of all patients received statins, whereas a minority of $10 \%$ received cholesterol-lowering medication other than statins. The control group and the intervention group did not differ in percentage of patients receiving cholesterollowering medication. During the 9-month period, however, the intervention group had a larger increase of prescribed dosage of statins ( 7.1 milligrams) than the control group ( 4.3 milligrams, $p=.06$ ). 
Table 3. Cholesterol-lowering medication at baseline and at 9-month follow-up in intervention and control group

\begin{tabular}{|c|c|c|c|c|c|}
\hline \multirow[t]{2}{*}{ Medication } & \multicolumn{2}{|r|}{ Baseline } & \multicolumn{2}{|r|}{9 months } & \multirow[t]{2}{*}{$n$} \\
\hline & $\%$ & $\begin{array}{l}\text { dose in mg/day } \\
(S D)^{\text {. }}\end{array}$ & $\%$ & $\begin{array}{l}\text { dose in mg/day } \\
\text { (SD). }\end{array}$ & \\
\hline \multicolumn{6}{|c|}{$\begin{array}{l}\text { All cholesterol-lowering } \\
\text { medication }\end{array}$} \\
\hline Intervention & 54.8 & $-c$ & 83.3 & & 84 \\
\hline Control & 51.2 & - & 86.9 & & 84 \\
\hline \multicolumn{6}{|l|}{ Statins } \\
\hline Intervention & 52.4 & $10.8(12.5)$ & 81.0 & $17.9(16.7)$ & 84 \\
\hline Control & 50.0 & $10.0(12.0)$ & 81.0 & $14.3(12.5)$ & 84 \\
\hline \multicolumn{6}{|l|}{ Gemfibrozil } \\
\hline Intervention & 4.8 & $57.1(257.1)$ & 8.3 & $85.7(337.7)$ & 84 \\
\hline Control & 2.4 & $21.4(145.7)$ & 8.3 & $100.0(333.7)$ & 84 \\
\hline \multicolumn{6}{|l|}{ Acapimox } \\
\hline Intervention & 0.0 & $0.0(0.0)$ & 1.2 & $3.0(27.3)$ & 84 \\
\hline Control & 0.0 & $0.0(0.0)$ & 1.2 & $3.0(27.3)$ & 84 \\
\hline \multicolumn{6}{|l|}{ Cholestyramine } \\
\hline Intervention & 0.0 & $0.0(0.0)$ & 0.0 & $0.0(0.0)$ & 84 \\
\hline Control & 1.2 & $0.1(0.9)$ & 1.2 & $0.1 .(0.9)$ & 84 \\
\hline
\end{tabular}

\footnotetext{
Note. "Mean dose (in mg/day) for entire control group or intervention group

bIncluded are only those patients with known lipid levels at baselene and at follow-up, i.e. only patients who are also included in the cholesterol analyses.

Dose prescriptions of all cholesterol-lowering medication can not be added.
}

There were no differences between both groups in dose prescriptions in any of the other cholesterol-lowering medications.

If the former ANCOVAs were corrected for change in prescribed dosage of statins from baseline to follow-up, the favorable effects of the intervention on reduction of total serum cholesterol reached a trend for significance, $F(1,160)=3.62, p=$ .06. In an analysis with the subgroup of patients with elevated baseline total cholesterol level, controlling for statin changes during follow-up did not alter the significance of the favorable effects, $F(1,75)=5.69, p=.02$. The effects of the intervention on LDL cholesterol changed slightly after controlling for statins in the total study population, $F(1,157)=2.05, p=.15$. For the subgroup of patients with high baseline cholesterol, however, the difference between intervention and control group remained significant, $F(1,75)=6.10, p=.02$. Controlling for statins in the HDL and triglyceride analyses did not change any of the results that were previously found. In the subgroup of patients with elevated cholesterol levels, after 
controlling for statins HDL cholesterol increased to a larger extent in the control group compared to the intervention group, $F(1,74)=5.22, p=.03$.

\section{Dielary fat consumption}

During follow-up, dietary fat consumption decreased in the intervention group by 15 grams per day (from 100 grams/day to 85 grams/day), whereas the control group showed a reduction of 8 grams per day (107 at baseline, 99 at follow-up). ANCOVA demonstrated that the difference between both groups was almost significant, $F(1,142)=3.71, p=.06$. The intervention was more effective in reducing dietary fat consumption in patients with lower baseline dietary fat intake than in patients with higher dietary fat intake at baseline, which was demonstrated by a significant interaction variable Group Allocation $x$ Baseline Dietary Fat Consumption $(p=.04)$. In patients with relatively low dietary fat intake at baseline (i.e. $\leq 80 \mathrm{grams} /$ day), the intervention group showed a reduction of dietary fat intake of $10 \%$, whereas the control group increased dietary fat intake with $28 \%, F$ $(1,141)=9.76, p=, 00$. In patients with high baseline dietary fat intake, no intervention effects were found. In this subgroup of patients, the intervention and control group reduced dietary fat intake with $16 \%$ and $14 \%$, respectively.

Change in dietary fat consumption did not contribute to a change in serum cholesterol, $F(1,146)=.00, \rho=98 . A$ posteriori-performed subgroup analyses, however, revealed that the relation between serum cholesterol levels and dietary fat consumption depended upon baseline fat consumption. Reduction in dietary fat consumption contributed significantly to a reduction, in serum cholesterol in the subgroup of patients with relatively low baseline dietary fat intake (i.c. $\leq 80$ grams/day, $n=48), F(1,45)=8.9, p=.01$. In patients with high baseline dietary fat intake (i.e. $>80 \mathrm{grams} /$ day), no relation was found.

\section{'Last observation carried forward' analyses}

During this study, 10 patients had missing lipid values at 9-month follow-up, equally divided among control group and intervention group. These values were replaced by the last observed value. For patients with missing values at baseline, missing data were replaced for the mean of all baseline values, provided that at least one follow-up mẹasurement was available.

The interyention remained effective in reducing total cholesterol levels with a trend for significance, $F(1,174)=3.54, p=.06$. The effects on LDL cholesterol for the total group were no longer significant, $F(1,174)=2.04, p=.16$, HDL cholesterol increased to a larger extent in the control group compared to the intervention group, $F(1,174)=8.24, p=.01$, and the changes in triglycerides did not differ between both groups, $F(1,174)=0.28, \rho=.60$. Controlling for statins did not lead to changes in these findings.

Fat consumption was no longer reduced to a larger extent in the intervention group compared to the control group, $F(1,170)=1.59, p=.21$, and adding the alterations in fat consumption from baseline to follow-up as a covariate to the 
analyses, revealed again that fat consumption did not contribute to the alterations in cholesterol levels. ${ }^{2}$

In the subgroup of patients with elevated baseline total cholesterol levels, the last observation carried-forward analyses did not reveal results that differed from the initial analyses. In this high lipid group, the intervention favorably altered total cholesterol, $F(1,87)=4.42, p=.04$ and LDL cholesterol levels, $F(1,87)=4.58, p$ $=.04$, and had no effect on HDL, $F(1,87)=0.94, p=.34$, and triglycerides, $F(1$, $87)=.07, p=.79$. Controlling for statins did not change these results.

\section{DISCUSSION}

Total serum cholesterol was significantly reduced in the intervention group compared to the control group. This effect was especially strong in patients who. needed the intervention the most: those with high baseline total cholesterol levels. LDL cholesterol was also reduced to a larger extent in the intervention group as compared to the control group. Again, the effects were strongest for patients with. high baseline LDL cholesterol. The intervention group had a larger increase of prescribed dose of statins than the control group, and correcting the analyses for this difference weakened the favorable effects of the intervention on I.DL. cholesterol and total cholesterol, although the favorable effects on total cholesterol still showed a trend for significance. In patients with high baseline cholesterol levels, thus in the patients who needed most, the intervention had a beneficial effect on total and LDL cholesterol irrespective of the taking of statins. Moreover, if missing values are considered and included in the analyses, the effects of the intervention on total and LDI, cholesterol in patients with high baseline choleserol levels continue to be significant.

How can the favorable reductions in total and LDL cholesterol in the intervention group be explained? The intervention focused on a reduction of dietary fat consumption, and this was reflected in the fact that dietary fat intake decreased to a larger extent in the intervention group as compared to the controls. This effect was particularly found in patients with already relatively low baseline dietary fat intake, but not in patients who consumed much dietary fat at baseline. Remarkably, the reduced dietary fat consumption was not related to altered serum cholesterol levels. Perhaps even more disturbing, we found a relation between reduced dietary fat consumption and favorably altered serum cholesterol levels in patients with relatively low dietary fat consumption at baseline. This relation was not found in parients with high baseline dietary fat intake, an effect that could not be explained by a restriction of range in the latter group. How can these puzzling results be explained? Patients who consumed much dietary fat to begin with, reduced their fat consumption irrespective of their group allocation. Moreover, in both groups

\footnotetext{
2In the initial analyses, change in dietary fat consumpuon from baseline to follow-up could not be added as a covariate to the analyses, due to many misstng data in dietary quesnonnastes In the last observarion carried-forward analvses missing data were less of a problem, so change in detary fat consumption from baseline to follow-up was added as a covanate th the analyses
} 
control and intervention - cholesterol levels are reduced, irrespective of group allocation. On the other hand, those who already consumed a healthy diet at entry in the study started eating even healthier after the intervention, and serum cholesterol levels are significantly reduced. Control patients who consume a healthy diet at entry in the study do not exert themselves to reduce their fat consumption any further, and serum cholesterol levels do not change.

Why is the reduced fat consumption after the intervention not associated with reduced cholesterol levels in all patients, but only in those with low baseline fat consumption? We can only speculate about explanations for this remarkable finding. Perhaps the self-reported dietary questionnaire was not reliable for the subgroup of patients with high baseline fat consumption, whereas it may have been reliable for patients with low baseline fat intake, because of which the true relation between favorably altered dietary habits and lowered serum cholesterol levels was obscured. Although a valid dietary questionnaire was used, it is quite conceivable that persons with high dietary fat intake may be less concerned with their eating habits; thus they may hardly realize what they consume, and consequently they may have difficulty filling out a dietary questionnaire reliably. Persons with low fat intake may ensure they eat a healthy diet, which makes them aware of precisely what they eat, which in turn makes it easier for them to fill out a questionnaire on dietary habits. Unfortunately, no studies are known concerning the difference in reliability of the dictary questionnaire between people who consume much and little dietary fat. Another speculation which might explain the obscure relation between fat consumption and cholesterol in our study may be that the reduction in fat consumption that is needed in those who consume much dietary fat in order to bring about lowered cholesterol levels, is much larger than the reduction in fat consumption they achieved.

What now may be the explanation for the fact that our intervention led to reduced cholesterol levels in those patients with elevated cholesterol levels, if the reduced fat consumption we reported is not responsible for this effect? A plausible explanation is that the intervention indirectly modified serum cholesterol levels by enhancing adherence to medication regimen. If patients in the intervention group were taking their cholesterol-lowering medication more consistently than control patients, this may account for the larger reduction of total and LDL cholesterol found in the intervention group. This may also explain the finding that the positive intervention effect is particularly found in patients with elevated cholesterol levels; after all, these are the patients with the highest dose of statins. Side effects (e.g., muscular pain, gastric or intestinal complaints) are dose-dependent, and perhaps patients are more willing to accept the adverse side effects of their medication after participation in the intervention. Unfortunately, no data were available on patients' compliance with medication regimen.

Could the favorable effects of the intervention on total cholesterol and LDL cholesterol be explained by regression to the mean, especially since the effects were found in the high-risk groups? We may indeed expect that high cholesterol levels at baseline will drop at follow-up measurements because of regression to the mean. Baseline cholesterol levels were, however, only a little bit higher in the intervention 
group than in the control group, a difference that was far from significant. Moreover, cholesterol levels of the intervention patients dropped to a lower level than in the control group, and this can not be accounted for by regression to the mean. So the favorable effects on total and LDL cholesterol can not solely be explained by regression to the mean. Moreover, regression to the mean can not explain the difference in intervention effects for the subgroup of patients with high baseline cholesterol. For this subgroup, baseline cholesterol levels were equally high in the intervention group and in the control group, and we may expect that cholesterol levels drop both in the intervention and in the control group. Although this is indeed what we found, we also saw that the reduction in the intervention group was larger than that in the control group. It is not so surprising though, that the effects were particularly found in patients with high baseline cholesterol. After all, one can hardly expect an intervention to gain a profit in patients with low cholesterol levels to begin with.

An unexpected finding was that although HDL cholesterol increased in both groups, the increase was larger in the control group. At 9-month follow-up, $81 \%$ of our study population received statins for cholesterol lowering, and it is known that statins raise $\mathrm{HDL}$ cholesterol. This does not, however, fully explain why the increase of HDL cholesterol was larger in the control group, since controlling for altered statin prescriptions did not change the results. A sound explanation yet has to be found. Perhaps patients in the control group increased their use of vegetables, fruit, whole grains or fish to a larger extent than patients in the intervention group, but data fail us to study this in detail. The unexpected finding that the increase of HDL cholesterol was larger in the control group than in the intervention group was not found in the subgroup of patients with elevated baseline levels. Controlling for statins, however, again showed a negative effect on the increase of HDL cholesterol in the intervention group. It should be noted though that the increase of prescribed statins in itself might be an effect of the intervention. As suggested before, it is possible that as a result of the intervention, patients in the intervention group are more willing to accept the unpleasant side effects of cholesterol-lowering agents, and consequently receive a higher dose of the drug.

\section{Limitations of the study}

Nearly $14 \%$ of all patients included in the study dropped out, which limits the study to a certain extent. If missing data for cholesterol values are included, about $22^{\circ} \%$ of patients could not be included in the analyses. Although this seems like a large dropout percentage, it should be noted that similar studies usually encounter comparable dropout rates (DeBusk et al., 1994; Engblom, Korpilahti, Hämäläinen, Puska, \& Rönnemaa, 1996; Van Elderen \& Dusseldorp, 2001).

A second limitation of the study is that we do not have any cost comparison data of our intervention and usual care, which makes it impossible to draw any conclusions about the cost-effectiveness of the progtam.

In general, current medication policies have large effects on cholesterol levels, because of which it may be hard to exert an additional favorable effect on 
cholesterol with a diet. In our study, $53 \%$ of all. patients were already receiving cholesterol-lowering drugs at entry in the study, which partly explains the rather favorable total serum cholesterol levels at baseline (a mean of $5.1 \mathrm{mmol} / \mathrm{L}$ or 197 $\mathrm{mg} / \mathrm{dl}$ for the total study population). In spite of the large proportion of patients with cholesterol-lowering medication, we were able to show an additional cholesterol lowering effect of the intervention concerning total cholesterol and LDL cholesterol. This favorable effect is important, not only because a major risk factor for CAD is reduced, but also because patients are shown that they can take control of their own health by following the advice given in the intervention program.

Thus, our short intervention was effective in reducing the cardiopathogenic total and L.DL cholesterol levels, especially in patients with elevated cholesterol levels, which may lead up to a cardioprotective effect in the long run. The positive indications of these findings are, however, restrained by an unexpected finding on the lower increase in HDL cholesterol levels in the intervention group compared with the control group. Studies on the relative risks of elevated LDL and lowered HDI. cholesterol report ambiguous results as to which is more important (Pfeffer, Sacks, \& Moyé, 1999; West of Scotland Coronary Prevention Study Group, 1998). Since the subgroup of patients who are in highest need of cholesterol lowering, i.e. those with high baseline cholesterol levels, profit from the intervention with reduced total and LDL cholesterol levels without marked negative side effects on HDL cholesterol, such an intervention may be advisable. The lower increase of $\mathrm{HDL}$ cholesterol in the intervention group, however, should make us cautious of advising the intervention to the general population of AMI and CABG patients under the age of 70 . 
APPENDIX TO CHAPTER 3: SECONDARY CLINICAL OUTCOME MEASURES 


\section{Introduction}

While cholesterol levels were considered primary outcome measures in this study, the effect of the intervention was also determined on secondary outcome measures, i.e. smoking, blood pressure, and Body Mass Index. The results concerning these secondary outcome measures are reported in Tables A through D. The main results will briefly be described in the general discussion of this thesis.

\section{Secondary clinical outcome measures}

Height of the patients was measured during the first outpatient visit, whereas weight, systolic and diastolic blood pressure were measured at baseline, three months after discharge, and at 9-month follow-up, and treated if indicated. From height and weight measures, Body Mass Index (BMI) was calculated. Self-report smoking habits were measured through a structured interview on lifestyle (Falger, $1989)$.

Table A. Results of Chi-square analyses comparing the intervention and control group on postintervention and 9-month follow-up smoking cessation rates, in which baseline smoking rate is used as a covariate ${ }^{a}$

\begin{tabular}{lccccc}
\hline \multicolumn{1}{c}{ Measure } & \multicolumn{3}{c}{ Intervention } & Control & \\
& $\%$ & $n$ & $\%$ & $n$ & $x$ \\
\hline Simoking & & & & & \\
Before incident & 47 & 89 & 43 & 81 & \\
Baseline & 15 & 89 & 9 & 81 & 2.46 \\
Post & 20 & 89 & 11 & 81 & 0.29 \\
9 months & 26 & 89 & 21 & 81 & \\
\hline
\end{tabular}

Note. *Covarates in these analyses were baseline smoking rate, gender, age,

"CABGivs, no C.ABG".

$p<05 . p<10$ 
Table B. Results of ANCOVA comparing the intervention and control group on postintervention and 9-month follow-up Body Mass Index (BMI), in which baseline BMI is used as a covariate

\begin{tabular}{lccccccc}
\hline \multicolumn{1}{c}{ Measure } & \multicolumn{3}{c}{ Intervention } & & Control & & \\
& $M$ & $S D$ & $n$ & $M$ & $S D$ & $*$ & $F$ \\
\hline Body Mass Index & & & & & & & \\
$(\mathrm{kg} / \mathrm{m} 2)$ & 26.5 & 3.2 & 80 & 26.3 & 3.4 & 81 & \\
Baseline & 26.5 & 3.3 & 80 & 26.5 & 3.5 & 81 & 0.66 \\
Post & 26.8 & 3.3 & 80 & 26.7 & 3.7 & 81 & 1.24 \\
9 months & & & & & & & \\
\hline
\end{tabular}

Note. "Covariates in these analyses were baseline BMI $\left(\mathrm{kg} / \mathrm{m}^{2}\right)$, gender, age, "CABG vs, no $C A B G "$, antidiabetic medication at baseline, baseline angina pectoris, baseline dyspnoca.

$* p<.05 .1 p<.10$

Table C. Results of ANCOVA comparing the intervention and control group on postintervention and 9-month follow-up blood pressure, in which baseline blood pressure level is used as a covariate

\begin{tabular}{|c|c|c|c|c|c|c|c|}
\hline \multirow[t]{2}{*}{ Measure } & \multicolumn{3}{|c|}{ Intervention } & \multicolumn{3}{|c|}{ Control } & \multirow[b]{2}{*}{$F$} \\
\hline & $M$ & $S D$ & $n$ & $M$ & $S D$ & $n$ & \\
\hline \multicolumn{8}{|l|}{ Systolic (mm Hg) } \\
\hline Baseline & 131.5 & 21.7 & 83 & 125.5 & 19.3 & 82 & \\
\hline Post & 129.2 & 18.4 & 83 & 128.3 & 19.4 & 82 & 0.91 \\
\hline 9 months & 131.7 & 18.4 & 83 & 133.2 & 18.6 & 82 & 2.59 \\
\hline \multicolumn{8}{|l|}{ Diastolic $(\mathrm{mm} \mathrm{Hg})$} \\
\hline Baseline & 82.7 & 10.2 & 83 & 79.9 & 10.8 & 82 & \\
\hline Post & 83.4 & 10.5 & 83 & 81.5 & 8.6 & 82 & 0.25 \\
\hline 9 months & 84.1 & 9.4. & 83 & 84.1 & 6.9 & 82 & 0.28 \\
\hline
\end{tabular}

Note "Covariates in these analyses were baseline blood pressure ( $\mathrm{mm} \mathrm{Hg}$ ), gender, age, "CABG vs. no CABG", blood pressure lowering medication at baseline.

$* p<.05 .9<.10$ 
Table D. Medication with an effect on blood pressure at baseline and at 9-month follow-up in intervention and control group

\begin{tabular}{|c|c|c|c|}
\hline Medication & $\begin{array}{l}\text { Baseline } \\
\%\end{array}$ & $\begin{array}{l}9 \text { months } \\
\%\end{array}$ & $\pi$ \\
\hline \multicolumn{4}{|c|}{ All blood pressure } \\
\hline Intervention & 91.9 & 86.0 & 83 \\
\hline Control & 88.0 & 84.3 & 82 \\
\hline \multicolumn{4}{|l|}{ Beta-blockers } \\
\hline Intervention & 74.7 & 53.0 & 83 \\
\hline Control & 65.9 & 43.9 & 82 \\
\hline \multicolumn{4}{|c|}{ Calcium antagonists } \\
\hline Intervention. & 15.7 & 9.6 & 83 \\
\hline Control & 11.0 & 6.1 & 82 \\
\hline \multicolumn{4}{|l|}{ ACE inhibitors } \\
\hline Intervention & 30.1 & 18.1 & 83 \\
\hline Control & 25.6 & 19.5 & 82 \\
\hline \multicolumn{4}{|l|}{ Diuretics } \\
\hline Intervention & 8.4 & 7.2 & 83 \\
\hline Control & 8.5 & 7.3 & 82 \\
\hline
\end{tabular}

Note. Included are only those patients with known blood pressure levels at baseline, postintervention and at 9-month follow-up, i.e. only patients who are also included in the blood pressure analyses.

\section{Medication with an effect on blood pressure}

During the 9-month period of follow-up, the intervention group had a larger decrease of prescribed dosage of captopril $(p=.04)$ and diltiazemhydrochloride $(p$ $=.09)$ than the control group. If the ANCOVAs were corrected for change in prescribed dosage from baseline to 9-month follow-up of these agents, the results for diastolic blood pressure remained the same. The 9-month effect of the intervention on systolic blood pressure, however, reached statistically a trend for significance in favor of patients in the intervention group; $F(1,157)=3.77, p=$ .05 . 
CHAPTER 4

DO META-ANALYSES OF THE EFFECTS OF PSYCHOEDUCATIONAL INTERVENTIONS FOR CORONARY PATIENTS STILL GIVE A VALID ESTIMATE OF THE EFFECT?

Submitted in revised form as:

Sebregts, E.H., Appels, A. and Bär, F.W. Do meta-analyses of the effects of psychoeducational interventions for coronary patients still give a valid estimate of the effect? 


\section{ABSTRACT}

Meta-analytic studies are sometimes conducted to estimate the effects of psychosocial interventions for patients with coronary artery disease (CAD) on cardiac mortality or morbidity. We studied the influence of secular trends on the effects of psychoeducational intervention studies for patients with CAD. Effects of psychoeducational interventions on cardiac mortality rates decrease in more recently published studies compared to older studies. The waning effect on mortality is not related to decreasing mortality rates in time. Instead, it seems to be associated with changes in the composition of the study samples. Because of this, meta-analytic studies, which include old samples, may lose their external validity. 


\section{INTRODUCTION}

Psychological interventions often aim to reduce mortality and morbidity rates in coronary artery disease (CAD) patients after hospitalisation, through improvement of lifestyle or by reducing psychological distress. Since many studies suffer from a lack of power to investigate effects on mortality, studies are often combined in a meta-analysis in order to increase power. Substantial improvements in cardiac care during the last 30 years, however, hamper the comparability of old and new studies. Mortality rates have been reduced and populations of recent studies are very likely to differ substantially from those in older studies. For instance, primary prevention efforts may have changed the smoking and cating habits of a population that participates in an intervention programme into a population of inveterate smokers or hardened dietary fat consumers. Therefore, older studies might lose their external validity. Due to changes in external validity through the years, one should question whether it is correct to combine old and new studies in a meta-analysis.

The goal of our study was to investigate the influence of secular trends on the effects of psychoeducational intervention studies. This paper tackles the following questions: 1) Are the reported effects of psychoeducational interventions on cardiac mortality rates and recurrent infarction rates stable over time? 2) If not, may this change be explained by a) changing cardiovascular mortality rates and changing rates of recurrent myocardial infarction, b) altering characteristics of study populations in the course of time?

\section{METHODS}

\section{Selection of studies}

To test the hypotheses, the data of the most recently published meta-analysis concerning the effects of psychoeducational programmes on cardiac mortality and morbidity rates in CAD patients were used. (Dusseldorp et al., 1999). Since the Dusseldorp et al. study included studies published before 1998, we additionally performed a MEDLINE and PSYCINFO computer search to identify studies concerning health education or stress management interventions published from 1998 until 2002.' Following Dusseldorp et al., three types of key words were used (Dusseldorp et al., 1999). The first type concerned patient characteristics: MI, hear surgery, coronary bypass, percutaneous transluminal coronary angioplasty (PTCA), CHD, beart disorder, and cartiovascular disorder. The second type of key word concerned the outcome variables: mortality, morbidity, blood pressure, cholesterol, overneight, weight, obesity, smoking, physical exercise, nutrition, food, anxiety, depression, anger, emotional distress, well-

\footnotetext{
' For detailed descriptions of health education or stress management interventions, the reader is referred to Dusseldorp et al. (Dusseldorp et al., 1999)
} 
being and quality of life. The third type of key word concerned the type of programme: cardiac rehabilitation, psychological or psychoeducational intenvention, education, stress management, training, therapy, counselling, and relaxation.

Following Dusseldorp et al,, we included only studies with patients who had experienced a cardiac event within the six months prior to the start of treatment (Dusseldorp et al., 1999). A cardiac event was defined as AMI, CABG, PTCA, or some combination of these. Studies reporting effects on emotional distress were included only if effects on risk factors, related behaviours, morbidity or cardiac mortality were also reported. Only relatively high-quality studies were included in our study, that is, studies that included a control condition or comparison condition (i.e., randomised experiments or quasi-experiments). Quasi-experiments were included only when samples were stratified or matched pairwise, or when a certain time period was used as an assignment rule for patients from the same hospital. For risk factors, related behaviours, and psychological variables, a pre-test measurement was required.

Forty-five studies concerning the effects of psychoeducational programmes for CAD patients were included in our study (37 reported in Dusseldorp et al., and 8 additional studies published from 1998 onwards). ${ }^{2}$ Of these, 8 studies could be included in our analyses concerning effects of interventions on mortality rates; 11 studies could be included in our analyses concerning effects of interventions on recurrent myocardial infarction rates (table 1). In addition, patient characteristics and inclusion criteria were studied in all 45 studies.

\footnotetext{
: The following studies were included: lbrahim et al., 1974; Rahe, O'Neil, Hagan, \& Arthur, 1975; Pozen et al, 1977: Fielding, 1979; Kallio, Hamälainen, Hakkila, \& Luurila, 1979; Karvetti, 1981; Theorell, 1982; Young, Kottke, McCall, \& Blume, 1982; Bengtsson, 1983; Eirdman \& Duivenvoorden, 1983: Mayou, 1983; Srvarajan et al., 1983; Stem, Gotman, \& Kaslow, 1983; Dracup et al., 1984: Horlick, Cameron, Firor, Bhalerao, \& Baltzan, 1984; Frasure-Smith \& Pnnce, 1985; Oldenburg, Perkins, \& Andrews, 1985; Salonen, Hamynen, \& Heinonen, 1985; Fnedman et al., 1986; Marshall, Penckofer, \& Lewellyn, 1986; Stransky, Kalin, Schwarzenbach, \& Schar, 1986; Hedback \& Perk, 1987; Munro, Creamer, Haggerty, \& Cooper, 1988; Oldenburg. Illan, \& lastier, 1989. Van Dixhoom, Duivenvoorden, Staal, \& Pool, 1989; Perk. Hedbäck, \& Engvall, 1990: Tavlor et al., 1990; Fridlund, Högstedt, Lidell, \& Larsson, 1991: Engblom et al., 1992: Mitsibounas, Tsouna-Hadis, Rotas, \& Sideris, 1992; Gilliss et al., 1993; DeBusk et al., 1994: Van Elderen et al., 1994; Van Elderen-Van Kemenade. 1994; Lunden, 1995; lones \& West, 1996; frusure Smith et al., 1997; Carlsson, 1998; Hofman-Bang et al, 1999; Wallner et al., 1999; Domelas, Sampson, Gray, Waters, \& Thompson, 2000; Higgins, Haves, \& McKenna, 2001: Stagmo, Westun, Carkson, \& Israelsson, 2001; Van Elderen \& Dusseldorp, 2001; Hajek, Taylor, \& Mills, 2002.
} 
Table 1. Methodological characteristics of studies with 12-month mortality and recurrent infarction endpoints:

\begin{tabular}{|c|c|c|c|}
\hline Study & Randomisation & Target population & $\begin{array}{l}\text { Baseline sample } \\
\text { size }\end{array}$ \\
\hline $\begin{array}{l}\text { Ruhe et al. } \\
1975\end{array}$ & $\begin{array}{l}\text { Random assignment to } \\
\text { control and treatments." }\end{array}$ & $\begin{array}{l}\text { Patents under } 60 \text { years of age surving ther first AMI, and likely to remain in } \\
\text { the hospital area following hospital discharge. }\end{array}$ & $60, \mathrm{C} 22, \mathrm{~T} 38$ \\
\hline $\begin{array}{l}\text { Kallio et al. } \\
1979\end{array}$ & $\begin{array}{l}\text { Random assignment to } \\
\text { contrul and treatment }\end{array}$ & All patients under 65 years who had been treated in hospital for AMI. & 375, C. 187, T 188 \\
\hline $\begin{array}{l}\text { Young ct } \\
\text { al } 1982\end{array}$ & $\begin{array}{l}\text { Non-tandom consecutrve } \\
\text { recrutment of control and } \\
\text { treatruent }\end{array}$ & $\begin{array}{l}\text { All patients } 65 \text { or younger admutted to the coronary unit with a first confirmed } \\
\text { AMI or having returned to work after previous AMI; absence of other drabling } \\
\text { or life threatening illness, employed or, if retured, following a physically actres } \\
\text { lifestyle; survided the acute phase of the AMI }\end{array}$ & 197: C100, T 97 \\
\hline $\begin{array}{l}\text { Bengesson } \\
1983\end{array}$ & $\begin{array}{l}\text { Random assignment to } \\
\text { control and treatment }\end{array}$ & 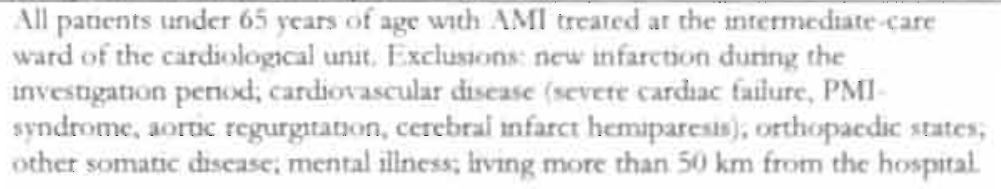 & 171: C $90, \mathrm{~T} 81$ \\
\hline $\begin{array}{l}\text { Stem et al. } \\
198.3\end{array}$ & $\begin{array}{l}\text { Random assignment to } \\
\text { cxercise. group counselling } \\
\text { and control group by } \\
\text { blocks of } 6 \text { patients }\end{array}$ & 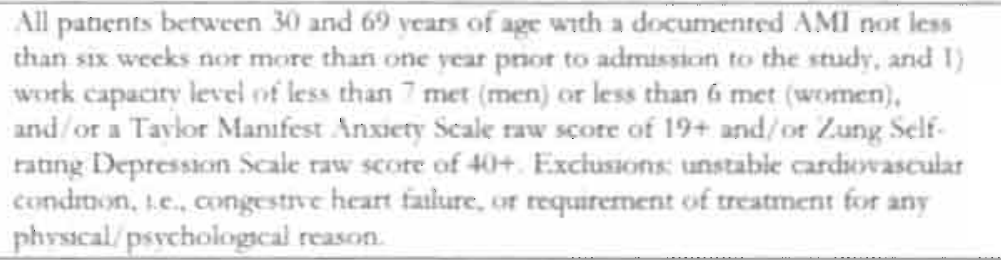 & $\begin{array}{l}\text { 106: Exerase } 42 \text {, } \\
\text { Counselling } 35, \text { C } 29\end{array}$ \\
\hline
\end{tabular}

Neur. $\mathrm{C}=$ Contrul; $\mathrm{T}=$ Treamen:

Problem After first year no randomisation to control group because of contamination of treatment control patients refused

- rable continues - 
Table 1 - continued

\begin{tabular}{|c|c|c|c|}
\hline Study & Randomisation & Target population & $\begin{array}{l}\text { Baseline } \\
\text { sample size }\end{array}$ \\
\hline $\begin{array}{l}\text { Frasure- } \\
\text { Smith and } \\
\text { Prnce, } 1985\end{array}$ & $\begin{array}{l}\text { Random assignment to } \\
\text { experimental and contrel } \\
\text { condituon in } 2 \text { groups: } \\
\text { medium risk or high risk }\end{array}$ & 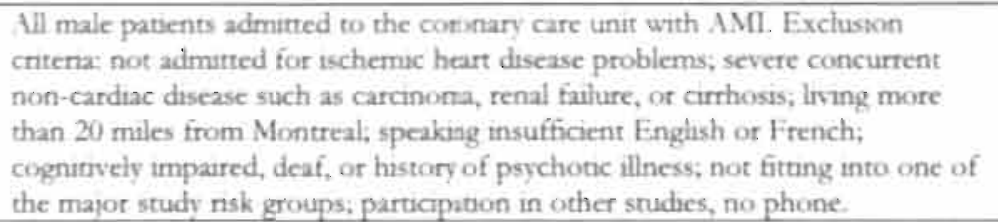 & $461: C 231, \mathrm{~T} 230$ \\
\hline $\begin{array}{l}\text { Finedman et } \\
\text { al. } 1986\end{array}$ & $\begin{array}{l}\text { Random assignment to } \\
\text { control or treatment }\end{array}$ & $\begin{array}{l}\text { All parients of } 64 \text { years of age or rotnger with one or more documented AMI } 6 \\
\text { months or earlier, who never smoked or que smoking for } 6 \text { months or longer, } \\
\text { and never been treated for exhubted stgns of diabetes mellinus. }\end{array}$ & 884: C 270. T 614 \\
\hline $\begin{array}{l}\text { Hedback } \\
\text { and Perk. } \\
1987\end{array}$ & $\begin{array}{l}\text { Treatment group } \\
\text { compared with non- } \\
\text { tandom control group } \\
\text { admined to the adjacent } \\
\text { hospital }\end{array}$ & $\begin{array}{l}\text { All survivang patients below } 65 \text { years of age who bad been admitted to the } \\
\text { hospital wath an AMI. }\end{array}$ & $305:$ C 158, T 147 \\
\hline $\begin{array}{l}\text { Frndhund et } \\
\text { al. } 1991\end{array}$ & $\begin{array}{l}\text { Random assignment to } \\
\text { control and treatment }\end{array}$ & $\begin{array}{l}\text { All panents aged } 65 \text { or younger with AMI, and with an independent living in the } \\
\text { hospital district after discharge from hospital, and a meaningful communicanon } \\
\text { and rehabilitation not hindered by the AMI or other serious illiness. }\end{array}$ & $110:$ C 58. T 52 \\
\hline $\begin{array}{l}\text { DeBusk et } \\
\text { al. } 1994 \text { : }\end{array}$ & $\begin{array}{l}\text { Random assignment to } \\
\text { control and treatment }\end{array}$ & $\begin{array}{l}\text { All parients aged } 70 \text { years or younget who were hospitalised for AMI. } \\
\text { Exclusions: death betore randomisaton, severe comorbid disease, sub- } \\
\text { stance abuse or other psychological problems; inability ro speak English. }\end{array}$ & 585: C 292, Т 293 \\
\hline $\begin{array}{l}\text { Jones and } \\
\text { West } 1996\end{array}$ & $\begin{array}{l}\text { Random assignment to } \\
\text { control and treatment }\end{array}$ & $\begin{array}{l}\text { All parients discharged home from bospital within } 28 \text { days of confirmed } \\
\text { myocardial infarction irrespectuve of age, sex, or previous cardiac history. } \\
\text { Exclusions: prolonged hospital stay lover } 28 \text { days) and discharge to long term } \\
\text { instimational care. }\end{array}$ & 2328: C 1160, T 1168 \\
\hline $\begin{array}{l}\text { Frasure- } \\
\text { Smuth et al } \\
1997\end{array}$ & $\begin{array}{l}\text { Random assignment to } \\
\text { control and treatment }\end{array}$ & $\begin{array}{l}\text { Patients with AMI nor related to a cotonary procedure. Exclusions: other life- } \\
\text { threatening conditons; residence more than } 20 \text { miles from study hospital; no } \\
\text { telephone; deafness; cognunve imparment, inability to speak English or French; } \\
\text { refusal by the patent's physician; participarion in other randomised trials. }\end{array}$ & 1376: C 684. T 692 \\
\hline
\end{tabular}




\section{Analyses}

Finst analyses: Are the reported effects of psycboeducational intenentions on cardiat mortality rates and recarrent myocandial infantion rates stable oter time?

To improve comparability of effects observed in different studies, we used only studies in which cardiac mortality rates or recurrent myocardial infarction rates were reported at 12-month follow-up. 'Spearman's rho correlation coefficient $\left(r_{i}\right)$ was computed between year of publication of the studies and the 12 -month odds ratios for cardiovascular mortality and recurrent infarctions. Twelve-month odds ratios were computed according to the method by Fleiss (Fleiss, 1994) (pp. 245260 , formula 17-18). If any cell frequency was zero, 0.5 was added to all cell frequencies. ${ }^{4}$ Higher odds ratios indicate larger favourable effects of the intervention on survival rates or on rates of recurrent myocardial infarction.

\section{Serond analyses: If effects of psycboeducational interventions on mortality and myocandial infartion rates are changing, may this trend be explained by a) changing mortality rates and changing rates of myocardial infantion; b) altering characteristics of study populations?}

a) Spearman's rho correlation coefficient $\left(r_{i}\right)$ was computed between year of publication of the studies and cardiovascular mortality rates. Next, Pearson's correlation coefficient ( $r)$ was computed between the mortality odds ratios and mortality rates. This was repeated for recurrent myocardial infarction rates.

b) In order to study trends in study population characteristics, we examined those characteristics that are reported in the majority of studies, i.e. mean age of the study population, percentage of women in the study, percentage of patients with one or more previous infarctions at entry in the study, and the percentage of patients with hypertension at entry in the study. Spearman's rho correlations were computed between year of publication of the studies and characteristics of the study population. Next, correlation coefficients were computed between the odds ratios for mortality and characteristics of the study populations. The analyses were repeated for recurrent myocardial infarctions. Subsequently, Spcarman's tho correlations were computed between year of publication of the studies and inclusion criteria of the studies.

\footnotetext{
'Studies with reported 12-month follow-up rates for mortality: Rahe et al., 1975, Kallio et al., 1979; Stern er al., 1983; Frasure-Smith \& Prince, 1985; Fnedman et al., 1986; Hedbäck \& Perk, 1987; DeBusk et al., 1994; Frasure-Smith et al., 1997. Studies with reported 12-month follow-up rates for recurrent myocardial infarctions: Rahe et al., 1975; Young et al., 1982; Bengtsson, 1983; Stern et al., 1983; Frasure-Smith \& Prnce, 1985; Friedman et al., 1986; Hedback \& Perk, 1987. Fndlund et al., 1991: DeBusk et al., 1994; Jones \& West, 1996; Frasure-Smuth et al., 1997.

"This was done in 2 studies included in the mortality analyses, and in 1 study that was included in the morbidity analyses
} 


\section{RESULTS}

Are the reported effects of psychoeducational interventions on cardiac mortality rates and on recurrent myocardial infarction rates stable over time?

In the 8 studies reporting 12 -month cardiac mortality rates, the year of publication of the studies correlates negatively with the odds ratios of cardiac mortality rates $(r$. $=-.79, p=.02$; figure 1). Thus, older studies reported larger favourable effects of interventions on cardiac mortality rates than more recently published studies.

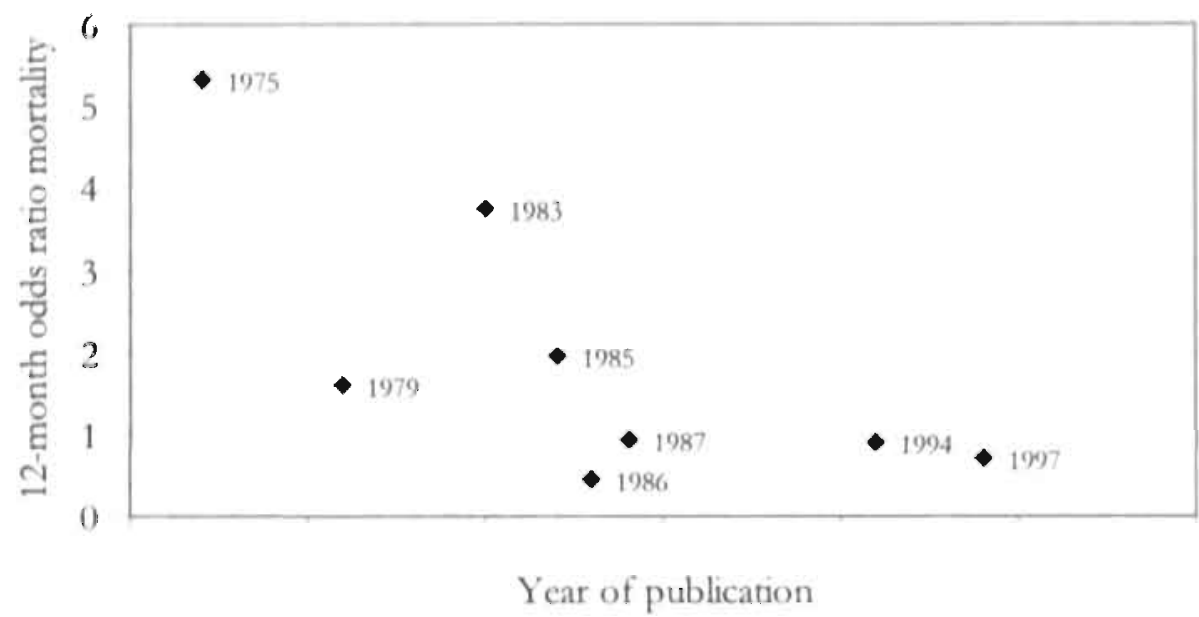

Figure 1. Correlation of cardiac mortality odds ratios at 12-month follow-up with year in which the study was published.

In the 11 studies reporting 12-month recurrent myocardial infarction rates, the year of publication of the studies does not correlate with the effect of the interventions on the rate of recurrent infarctions $\left(r_{i}=-.04, p=.91\right)$.

May the larger favourable effects of interventions in older studies be explained by changing cardiovascular mortality rates or recurrent myocardial infarction rates?

Cardiovascular mortality rates of all 8 studies are shown in table 2. Mortality rates seem to decline in the course of time. Spearman's tho correlation coefficient between year of publication and mortality rate (expressed as a percentage) is negative, but not significant $\left(r_{i}=-.55, p=16\right)$. Moreover, the odds ratios for mortality are not correlated with mortality rates $(r=.01, p=.98)$. Thus, higher or lower mortality rates in the studies are not associated with larger or smaller effects of interventions on mortality. 
Twelve-month recurrent infarction rates of all studies are reported in table 2 . The year in which the studies were published was not correlated with the 12-month recurrent infarction rates $\left(r_{r}=.03, p=.94\right)$.

Table 2. Twelve-month cardiovascular mortality and recurrent infarction rates in the control groups of all studies, in percentage

\begin{tabular}{lccc}
\hline Study & $\begin{array}{c}\text { Year of } \\
\text { publication }\end{array}$ & $\begin{array}{c}\text { \% Cardiovascular } \\
\text { mortality }\end{array}$ & $\begin{array}{c}\text { \% Recurrent } \\
\text { infarctions }\end{array}$ \\
\hline Rahe et al. & 1975 & 4.8 & 19.1 \\
Kallio et al. & 1979 & 14.0 & \\
Young et al. & 1982 & & 40 \\
Bengtsson & 1983 & 3.5 & 6.3 \\
Stern et al. & 1983 & 9.6 & 3.5 \\
Frasure-Smuth and Prnnce & 1985 & 0.9 & 9.6 \\
Friedman et al. & 1986 & 7.8 & 16.5 \\
Hedbäck and Perk & 1987 & 3.4 & 23.0 \\
Fndlund et al. & 1991 & & 6.9 \\
DeBusk et al. & 1994 & 3.4 & 4.5 \\
Jones and West & 1996 & & 5.0 \\
Frasure-Smith et al. & 1997 & & \\
\hline
\end{tabular}

May the larger favourable effects of interventions in older studies be explained by altering characteristics of study populations?

In the mortality analyses, we found that characteristics of the study populations from more recent studies differ from patient characteristics in older studies. With a trend for significance, patients in more recent studies are older $\left(r_{i}=.69, p=.06, \mathrm{~N}\right.$ $=8$ ), and more recent studies consist of a higher percentage of hypertensive patients $(r=.71, p=.07, \mathrm{~N}=7)$. Moreover, in recent studies there is a higher percentage of patients who are female $\left(r_{i}=.62, p=.10, N=8\right)$, and have had one or more previous infarctions at entry in the study $\left(r_{i}=.54, p=.27, N=6\right)$, but these correlations are not significant. The odds ratios for cardiac mortality correlate significantly and negatively with the mean age of the study population $(r=-71, p$ $=.05, \mathrm{~N}=8)$ and the percentage of patients with previous. AMI $(r=-.96, p=.00$, $N=6$ ), and correlate with a trend for significance with the percentage of hypertensive patients $(r=-.70, p=.08, N=7$ ). Thus, larger effects on mortality are found in studies with younger patients, fewer patients with previous myocardial infarction, and fewer patients with hypertension.

In the analyses for recurrent myocardial infarctions, more recent studies are characterised by older study populations as well $\left(r_{1}=.78, p=.01, N=10\right)$, and a higher percentage of women $\left(r_{i}=.65, p=.03, n=11\right)$. The odds ratios for recurrent infarctions correlate significantly and negatively with the percentage of patients with previous myocardial infarction $(r=-89, p=.00, N=9)$, and correlate with a trend for significance with the mean age of the study population ( $r$ $=-60, p=.07, N=10$ ). Thus, larger effects of interventions on recurrent 
myocardial infarction rates are found in studiẹ with younger patients and studies with fewer patients who had previous myocardial infarctions.

Which changes in population characteristics are revealed if all 45 studies are analysed? In this larger sample, we found that CAD patients included in more recently published studies are on average older than patients participating in older studies $\left(r_{i}=.37, p=, 02, n=38\right)$. Furthermore, more recently published studies are characterised by a higher percentage of women compared to older studies $\left(r_{s}=.50\right.$, $p=, 00, N=44$ ), and there are indications of a larger proportion of patients with a history of previous myocardial infarction(s) at entry in the study $\left(r_{\mathrm{s}}=.32, p=.12\right.$, $N=25$ ).

In more recently published studies, no inclusion criterion is used at all, or inclusion criteria in reference to age are higher compared to older studies. We found a significant correlation between year of publication of the study and the age criterion applied $\left(r_{t}=-45, p=, 00, N=43\right)$, in which age criterion was distributed evenly in 5 -year steps (i.e., 'no inclusion criterion concerning age', ' $\leq 80$ years of age', ' $\leq 75$ years of age', etc.). In addition, whereas older studies often include men only, more recent studies apply no exclusion criteria for women $\left(r_{t}=-.33, p=.03\right.$, $N=45$ ). If we consider only studies without inclusion criteria with reference to male sex, we find again a positive correlation between year of publication and percentage of women in the study $\left(r_{i}=.40, p=.02, n=36\right)$. If we consider only studies without inclusion criteria with reference to gender and age, no significant correlations are found between year of publication and mean age of the study population and percentage of women in the study. Moreover, there is an indication that older studies more often select patients with a first myocardial infarction than more recently published studies, which may select patients with a history of myocardial infarction $(\mathrm{s})\left(r_{i}=-.27, p=.08, N=43\right)$.

\section{DISCUSSION}

The effect of psychoeducational intervention programmes for AMI patients on cardiac mortality decreases significantly over time, as was demonstrated in the studies reporting 12 -month cardiac mortality rates. This decline of the effectiveness of these programmes cannot be attributed to lower mortality rates.

What phenomenon then may account for this trend? Researchers are increasingly less inclined to apply age limits in their studies, or they readjust these limits upward to include older patients. In addition, more and more studies include women, whereas in older studies solely men were included. We found shifts in patient characteristics from young, male patients in older studies to older patients with a larger proportion of women in more recently published studies. Moreover, there are indications that the study participants are shifting towards a 'sicker' population consisting of more patients with a history of myocardial infarction. The increasing age of the participating patients and the higher percentage of patients with 
previous myocardial infarctions are correlated with the waning effect of interventions on mortality.

How may this be explained? The changing patient characteristics may affect the scope of the interventions. Interventions used to be designed for and directed at the improvement of health status in young men. Are the contents of the interventions still valid to yield an effect in the increasingly older and more often female patients? Several recent studies show that it is precisely women that do not respond positively - in some cases even negatively! - to psychological interventions (Frasure-Smith et al., 1997; Sebregts, 2003). It might be that intervention programmes need more adaptation to major patient characteristics such as gender and age.

One may expect that the quality of psychoeducational interventions would improve based on experience gained from the past. Consequently, we would expect the effects of more recent interventions to be larger, not lower. It is also true, however, that lifestyle interventions that were found to be effective in earlier studies are nowadays integrated in standard cardiac rehabilitation. Control groups in psychoeducational intervention studies often receive standard exercise rehabilitation that contains many components also used in behaviour change therapies and stress management programmes. Because of this, more recent studies may contain more inveterate smokers or hardened dietary fat consumers than older studies.

Contrary to our expectations, we did not find a decreasing effect of psychoeducational intervention programmes for AMI patients on recurrent infarction rates over time. Neither did we find a trend of decreasing recurrent infarction rates over time. We did find changes in patient characteristics over time toward older and mote often female patients. But in spitc of the finding that the year of publication of the studies included in the recurrent myocardial infarction analyses is negatively correlated with age, the intervention effects on recurrent infarctions do not wane in the course of time. This phenomenon is not so casy to account for.

We speculate that the effect of the interventions on recurrent myocardial infarctions in recent studies might partly be produced by patients' improved adherence to medication. This effect will probably be larger in more recent studies than in older studies because of increased use of drugs, and may have a larger effect on the rate of recurrent myocardial infarctions than on cardiac mortality. When the older studies in our sample were conducted, medical treatment was predominantly directed at the treatment of complications caused by the AMI from which the patient was suffering. Thrombolytic therapy, percutaneous coronary intervention, and other medical treatments, which are increasingly popular, were aimed at limiting infarct size, and this way preventing mortality as a consequence of the AMI. Drugs that prevent or diminish the chances of a recurrent cardiac event gained ground in later years, with the introduction of drugs such as betablocking agents, statins, or ASA. This may be illustrated by a random recent report from the GISSI-group, where $44 \%$ of patients had $\beta$-blockers at baseline, $47 \%$ had ACE inhibitors and $91 \%$ had antiplatelet drugs to start with (Marchioli et al., 
2002). Consequently, if the effect of psychoeducational interventions is partly related to improved adherence to medication, the interventions in more recent studies may be effective in reducing myocardial infarctions.

\section{Limitations of the study}

This study has some weaknesses. The precision of our estimates may have been affected by focusing on publication year. It would have been more accurate to use the years in which studies were actually conducted, but in some of the studies this information was not reported. We did, however, redo the mortality analyses using the starting year of the study and replaced the (one) missing study-year by the year of publication minus mean delay until publication for all studies. The results of these secondary analyses showed again a negative correlation between study year and decreasing effect on mortality.

We studied the trends in effects of psychoeducational interventions on mortality and recurrent myocardial infarction rates in a limited number of studies, since only a few studies reported 12 -month mortality and morbidity rates. The resulting small power of our analyses prescribes caution in drawing firm conclusions from our results. Nevertheless, we had sufficient power to demonstrate changing characteristics of study populations over time.

Another factor we should bear in mind is that the methodological quality of more recently conducted studies generally exceeds the quality of older studies. We tried to preclude this possibility by including only relatively high-quality studies in our analyses. We are unable to pronounce upon qualitative factors concerning the realisation of the study design and contents of the interventions, since methodology sections of publications often lack detailed descriptions. Some uncertainties in the measurement and definition of AMI and hypertension in studies also limit our study, which warrants caution in interpreting correlations concerning these variables. This does not alter the findings in reference to age and gender of the study populations over time, however.

Finally, we focused the analyses on cardiac mortality and recurrent infarctions at 12-month follow-up, whereas differentiation of cardiac mortality and morbidity rates might start at a later stage after the cardiovascular incident. This turned out to be impossible to study since very few publications on psychoeducational intervention studies report cardiac mortality rates at uniform long-term follow-up moments.

The results of this study show that meta-analytic studies are losing their external validity. The estimate they give about the effects of an intervention on cardiac mortality and morbidity is becoming invalid. unless it is corrected for changes in the composition of the study populations. 


\section{CHAPTER 5}

\section{IS IT USEFUL TO SELECT CORONARY ARTERY DISEASE PATIENTS FOR A MULTIFACTORIAL BEHAVIORAL GROUP INTERVENTION?}

Submitted as:

Sebregts, E.H.W.J., Falger, P.R.J., Kester, A.D.M., Appels, A. and Bar, F.W.H.M. Is it useful to select coronary artery disease patients for a multifactorial behavioral group intervention? 


\section{ABSTRACT}

It is still unclear whether the efficiency of cardiac rehabilitation can be improved by pre-selection of patients. The primary aims of this study were therefore to examine the quality of a screening device that was developed to select patients for a multifactorial intervention, and to determine whether selected patients profited more from a multifactorial intervention than patients, who did not meet the selection criteria. Two hundred and one acute myocardial infarction patients or patients who underwent coronary artery bypass grafting were randomized to a multifactorial behavioral group intervention or to a usual care control group. All patients were screened in order to select patients with an unfavorable risk factor profile for coronary artery disease. Results confirmed. the validity of the selection device. The hypothesis that patients with an unfavorable risk profile would profit more from the intervention than patients with a favorable profile was tested by including the interaction Group Allocation x Profile in the analyses computing the effect of the intervention on clinical and psychological risk factors. The effect of the intervention did not differ between those with or without a poor profile. Posthoc analyses showed that two of the eight risk factors studied changed significantly in the group with an unfavorable risk profile, while another three risk factors were improved with a trend for significance. In the group with a favorable profile one risk factor changed significantly. It is concluded that restricting an intervention program to patients with an unfavorable risk factor profile may increase the efficiency of cardiac rehabilitation. 


\section{INTRODUCTION}

Marked improvements in the survival of acute myocardial infarction (AMI) patients during the last 20 years have led to a growing number of AMI patients sent to cardiac rehabilitation. Traditionally, patients suffering from AMI were referred to physical exercise therapy in order to improve or restore their physical condition. Initially, this was needed because patients were immobilized for 4-6 weeks. However, due to the short admission times today ( \pm 1 week) and the carly mobilization after 1-2 days, physical condition is not hampered in most patients. Later on, efforts were added to restore psychological well-being. Nowadays, cardiac rehabilitation is becoming increasingly a multifactorial activity. Physical exercise is supplemented with psychosocial and lifestyle modifications through education, counseling and behavior interventions. Studies that evaluate psychosocial rehabilitation interventions for coronary artery disease (CAD) patients do not always report favorable effects, however (Frasure-Smith et al., 1997; Jones \& West, 1996).

The limited effectiveness of today's programs may be attributable to the fact that standard programs are offered to all patients, irrespective of whether or not they are in need of such a program. Since some subgroups would probably profit more than others, the selection of patients should be more focused. International guidelines for cardiac rehabilitation stimulate individually tailored programming British Association for Cardiac Rehabilitation, 1995; Corstiens, Kuijs-Wouters, Bär, \& Falger, 1999; Nederlandse Vereniging voor Cardiologic en de Nederlandse Hartstichting, 1995). Studies that evaluate the merits of pre-selection for cardiac rehabilitation are lacking, however. The aim of the present study was to determine the validity of a screening procedure developed at the University Hospital Maastricht in the Netherlands, and to determine whether patients selected by this procedure profit more from a multifactorial intervention than patients who do not meet the selection criteria.

\section{METHODS}

\section{Screening and selection of patients with a poor risk factor profile}

A multidisciplinary team, consisting of a physiotherapist, a cardiologist and' a psychologist, used two screening questionnaires in order to select patients who were likely to benefit from the intervention, planned on the basis of risk assessment and individual need. The two screening questionnaires had been developed at the University Hospital Maastricht, and were complemented with existing questionnaires (Corstiens et al., 1999).' The first screening questionnaire was taken during hospitalization, and the second several weeks later, after

\footnotetext{
'Selection of patients for the mulufactorial intervention was only one of several purposes of the screening questionnaires. Addinonal purposes were selection of patients for the physical training program, and evaluation of progress duning the physical training program.
} 
discharge from hospital. Both questionnaires contained items conceming diagnosis, risk factors for $\mathrm{CAD}$, demographic information, and emotional state. A more detailed description of their contents is depicted in table 1.

Based on both screening moments, each patient was screened by the multidisciplinary team in order to assess whether selection criteria were met that would select patients who were most in need of cardiac rehabilitation. This selection procedure was performed blinded for the patients' names. The selection criteria were developed such that patients who were assumed to have a poor risk. factor profile for $\mathrm{CAD}$ were distinguished from patients who were assumed to have a favorable risk factor profile for CAD. Therefore, patients that were selected are hereafter indicated as 'poor profile' patients, whereas non-selected patients are indicated as 'favorable profile' patients. Patients were considered poor profile patients if they met one of the following criteria: (1) possessing more than one modifiable risk factor for $\mathrm{CAD}$, without clear improvement in those risk factors after discharge from hospital; (2) unfavorable emotional profile during hospitalization and no improvement after hospital discharge as indicated by five or more negative self-descriptions at the Visual Analog Scale (table 1).

\section{Participants and procedure}

All patients admitted to the University Hospital Maastricht during the period of February 1996 through November 1997 were identified as eligible for the study by a team of physiotherapists working at the hospital's physiotherapy department. These patients were referred to them by cardiologists and cardiac surgeons, for physical training following AMI or coronary artery bypass grafting (CABG). Patients included were less than 70 years of age, who were admitted to the hospital with a confirmed diagnosis of AMI, CABG, or both, and who were able to participate in the regular physiotherapy exercise-program starting early after hospital discharge. The number of patients included in this study was based on feasibility within the time limits of the study. Patients were excluded if they were non-Dutch speaking, illiterate, or were currently suffering from any psychiatric disorder that would severely disturb participation in the intervention. During this 21 -month period, signed informed consent was given by 204 patients $(67 \%$ of all eligible patients).

After informed consent was given, patients were randomly assigned to either the intervention or to a usual care control group. This was independent of patient profiles. Borh groups received usual medical care, which consisted of regular medical check-ups by a cardiologist, and postdischarge exercise training sessions. 
Table 1. Contents of the screening questionnaires administered during hospitalization and after discharge

\begin{tabular}{|c|c|}
\hline Screening during hospitalization & Screening after discharge \\
\hline $\begin{array}{l}\text { - Cardiac history, cardiac and physical complaints, medication } \\
\text { - Risk factors: } \\
\text { Diabetes, bliood pressure, serum cholesterol level, } \\
\text { weight, earing habits, smoking habits, alcohol } \\
\text { consumptoon, hereditary defect of coronary artery } \\
\text { disease }\end{array}$ & $\begin{array}{l}\text { Cardiac and physical complants, medication } \\
\text { Risk factor changes (after hospital discharge): } \\
\text { W eight, caung habits, smoking habits, alcohol } \\
\text { consumptoon }\end{array}$ \\
\hline $\begin{array}{l}\text { - Patient's view of factors that contributed to his or her } \\
\text { cardiosascular complamts. Factors that the patient } \\
\text { wishes to change. }\end{array}$ & - Risk factors that the patient is currently changing \\
\hline $\begin{array}{l}\text { Acrivity level (past } 6 \text { months) } \\
\text { Demographic: } \\
\text { Educanon, employment }\end{array}$ & - Actrvty lesel (current) \\
\hline $\begin{array}{l}\text { Emotional state: } \\
\text { Visual Inahg Seale with } 1^{-} \text {patrs of opposite } \\
\text { adpectuves concerning emonons, c.g 'insecure-secure', or } \\
\text { exhausted-fresh' (dunng hospitalization) } \\
\text { HPPQ (past } 3 \text { months) }\end{array}$ & 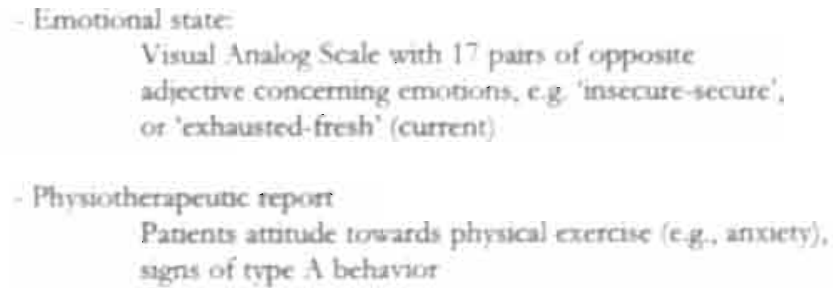 \\
\hline
\end{tabular}

Nis. $H P P Q=$ Heart Panents Psychological Questionnatre (Durch version: MPVH. Exdman, 1981). 


Admission:
Informed consent and
randomuzaton to
interventom or usual
care control group.
Administramon of first
screenung questionnaire.
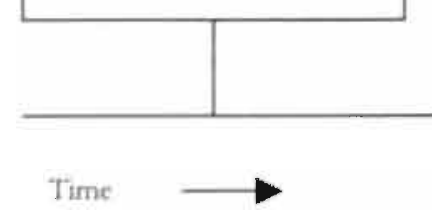

Time

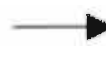

0 weeks
Administration of 2nd screening questionnaire.

Ist Interview and

questionnaires of

lifestrle and

psychological variables

1st Clinical

measurement.
Mulindisciplinary team selection of poor profile and favorable profile patients.

Start of interverition end of intervention.

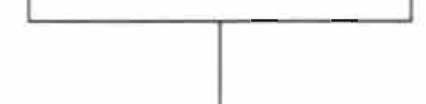

4.8 weeks $-12-16$ wecks
Last interview and questionnaires.

Last clinical

measurement.

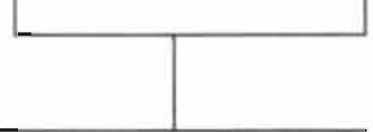

12 monrhs

Figure 1. Schema of the study procedure in time. 
In addition, patients that were assigned to the intervention group enrolled into a multiple risk modification program for secondary prevention of CAD. The selection of poor profile and favorable profile patients was performed independently of group assignment, after the second screening questionnaire was administered. Figure 1 presents the flow chart of the study procedure. Psychological measures and clinical data for the intervention groups were assessed (1) at baseline, which was approximately four weeks after hospital discharge (hereafter indicated as 'Baseline'), and (2) nine months after termination of the intervention, which was approximately one year after hospital discharge (9-month follow-up'). ${ }^{2}$ Measures for control patients were collected at comparable moments in time. Clinical data were obtained from the medical files, blinded to the patients' group allocation.

\section{Intervention}

Patients allocated to the intervention group were offered a combined stressmanagement, risk factor modification and health education program during eight weekly $2.5 \mathrm{hr}$-sessions, starting within 3 to 8 weeks after hospital discharge.' The therapists and cotherapists of the intervention were not aware of the outcome of the selection procedure. The contents of the intervention were primarily based on the methods of three major long-term clinical trials, the Recurrent Coronary Prevention Project (Bracke \& Thoresen, 1996), Project New Life (Burell, 1996), and the Lifestyle Heart Trial (Ornish et al., 1990), adapted to a format that would be feasible within the context of a short intervention program. During the sessions, 6 to 10 cardiac patients and their partners (whose participation was encouraged) were informed about CAD risk factors and risk factor modification by a multidisciplinary team. The group sessions were primarily educational, focusing on relevant information about specific topics concerning the development and course of CAD: e.g., the importance of a healthy non-fat diet, low serum cholesterol level, physical exercise, or risk of stress and type A behavior/hostility. Each session was concluded with breathing and relaxation exercises based on the work of Van Dixhoorn (Van Dixhoorn, 1997).

\section{Measures}

The following outcome measures were used: A. Physical clinical measures 1) Total serum cholesterol was measured during outpatient clinical visits. 2) Systolic blood pressure was measured during outpatient clinical visits. 3) Weight was measured during outpatient clinical visits. Body Mass Index (BMI) was calculated from weight and heigth. 4) Self-reported smoking habits were measured in a structured interview on lifestyle (Falger, 1989). B. Psychological measures 5) Type A Behavior was measured using a Dutch version of the Videotaped Structured Interview (VSI) (Friedman \& Powell, 1984), 6) Vital exhaustion was measured by

\footnotetext{
$2 \mathrm{~A}$ postuntervention measurement was performed immediately after the intervenuon, which was approximately three months after hospital discharge, but will not be studied in this paper

'For a more detailed description, see Sebregts et al., 2003
} 
the Maastricht Interview for Vital Exhaustion (MIVE)(Meesters \& Appels, 1996a). A score of $\geq 8$ is considered positive for vital exhaustion. 7) Anxiety was measured by administering a Dutch version (ZBV) of the Spielberger State-Trait Anxiety Inventory (STAI) (Spielberger et al., 1970; Van der Ploeg et al., 1980). 8) Quality of life was determined by a Dutch translation of the Multidimensional Index of Life Quality (MILQ) (Avis et al., 1996). The total score was computed by taking the unweighted sum of all scores.

\section{Data analysis}

\section{Preplanned hypothesis}

(1) The selection procedure makes no valid distinction between selected poor profile patients and non-selected favorable profile patients. This hypothesis was tested by comparing selected poor profile patients with non-selected favorable profile patients in reference to major clinical and psychological risk factors for CAD (i.e. cholesterol level, blood pressure, body mass index, smoking, type A behavior, vital exhaustion, anxiety and quality of life), using chi-square analyses and t-tests.

(2) Selected poor profile patients do not profit more from the intervention than non-selected favorable profile patients do. To test this hypothesis an interaction variable was computed, i.e. Group Allocation (control or intervention) x Profile (poor or favorable profile). The interaction variable was subsequently included in the ANCOVAs that were used before to study the effects of the intervention. The effects of the intervention on clinical and psychological risk factors were studied previously, and are or will be reported elsewhere (Sebregts, Falger, Bär, Kester, \& Appels, submitted; Sebregts, Falger, Appels, Bär, \& Kester, 2003). Covariates in all analyses were baseline risk factor level, gender, and age. Additional covariates in analyses of clinical risk factors were medication at baseline and "CABG vs. no CABG", since risk factor levels can be temporarily altered as a consequence of a CABG operation itself. Baseline angina pectoris and baseline dyspnea served as supplementary covariates in the BMI analyses. An additional covariate in the analyses of psychological factors was "AMI versus CABG only", because of the possible influence an AMI experience might have on the psychological outcome measures.

\section{Post boc anahyses}

Post-hoc analyses were used to compare changes in risk factors in the intervention group and the control group in poor and favorable profile groups separately. ANCOV Is were used to study the following questions: a) Do poor profile patients profit from the intervention as compared with poor profile patients allocated to the control group? b) Do favorable profile patients profit from the intervention as compared with favorable profile patients in the control group? 


\section{RESULTS}

\section{Dropouts}

A total of 304 patients were approached for the study, of which 204 patients (67 $\%$ ) were willing to participate. Twenty patients dropped out after hospital discharge but before baseline psychological measurements. These patients were not aware of group allocation.

\section{Hypothesis 1. Validity of the selection procedure}

Of the 204 parients, $114(57 \%)$ were classified as 'poor profile', and $87(43 \%)$ were identified as 'favorable profile'. Three patients could not be screened due to missing screening questionnaires.

Selected poor profile patients differed from non-selected favorable profile patients on all studied risk factors, with the exception of cholesterol and type $A$ behavior (table 2).

Table 2. Baseline measurements concerning clinical risk factors and psychological characteristics of non-selected (favorable profile) and selected (poor profile) patients

\begin{tabular}{lllll}
\hline Measurement & Not selected [SD] & $n$ & Selected [SD] & $n$ \\
\hline Total cholesterol $(\mathrm{mmol} / \mathrm{L})$ & $5.1[1.2]$ & 81 & $5.2[1.1]$ & 105 \\
Systolic blood pressure $(\mathrm{mm} / \mathrm{Hg})$ & $124.6[18.6]$ & 81 & $129.9[21.0]^{*}$ & 105 \\
Body Mass Index $\left(\mathrm{kg} / \mathrm{m}^{2}\right)$ & $25.5[3.1]$ & 81 & $27.3[3.3]^{*}$ & 109 \\
Smoking (\%) & 6.3 & 80 & $18.3 *^{*}$ & 104 \\
Type A behavior (VSI) & $208.3[60.4]$ & 80 & $220.8[56.9]$ & 104 \\
Vital Exhaustion (MIVE) & $3.9[3.3]$ & 80 & $6.8[5.3]^{*}$ & 103 \\
State Anxiety (STAI) & $35.6[9.1]$ & 75 & $40.8[10.7]^{* *}$ & 94 \\
Quality of Life (MIL.Q) & $192.1[27.2]$ & 77 & $172.5[29.4]^{* *}$ & 96 \\
\hline
\end{tabular}

Note. VSI $=$ Videotaped Structured Interview; MIVE $=$ Maastricht Interview for Vital Exhaustion; STAI = State Trait Anxiety Inventory; $M I L Q=$ Multidimensional Index of Life Quality; Ranges type A behavior 2-358; Vital Exhaustion 0-23; State anxiety 20-80; Quality of Life 35-245.

${ }^{\oplus} p<.10 ; * p<.05 ; * p<.01$

It should be noted that at the time the study was conducted, cholesterol values were not always known at the time of hospital discharge. Cholesterol levels could therefore not accurately be taken into account at the time of screening. Moreover, the physiotherapist's judgement concerning signs of type A behavior was often lacking in the screening questionnaire. 
There was no significant difference between poor profile patients and favorable profile patients concerning medication. Of all poor profile patients, $54 \%$ received cholesterol-lowering medication at baseline, as opposed to $46 \%$ of favorable profile patients $(p=31)$. Ninety-three percent of the poor profile patients received medication with an effect on blood pressure, versus $88 \%$ of the favorable profile patients $(p=.31)$.

\section{Hypothesis 2. Selected poor profile patients do not benefit more from the multifactorial intervention than non-selected favorable profile patients do}

The distribution of poor profile and favorable profile patients among the intervention and control group is reported in table 3. Baseline risk factor measures and changes in those measures at follow-up for poor profile and favorable profile patients in the intervention and control group are shown in table 4.

Table 3. Distribution of poor profile patients and favorable profile patients among intervention and control group

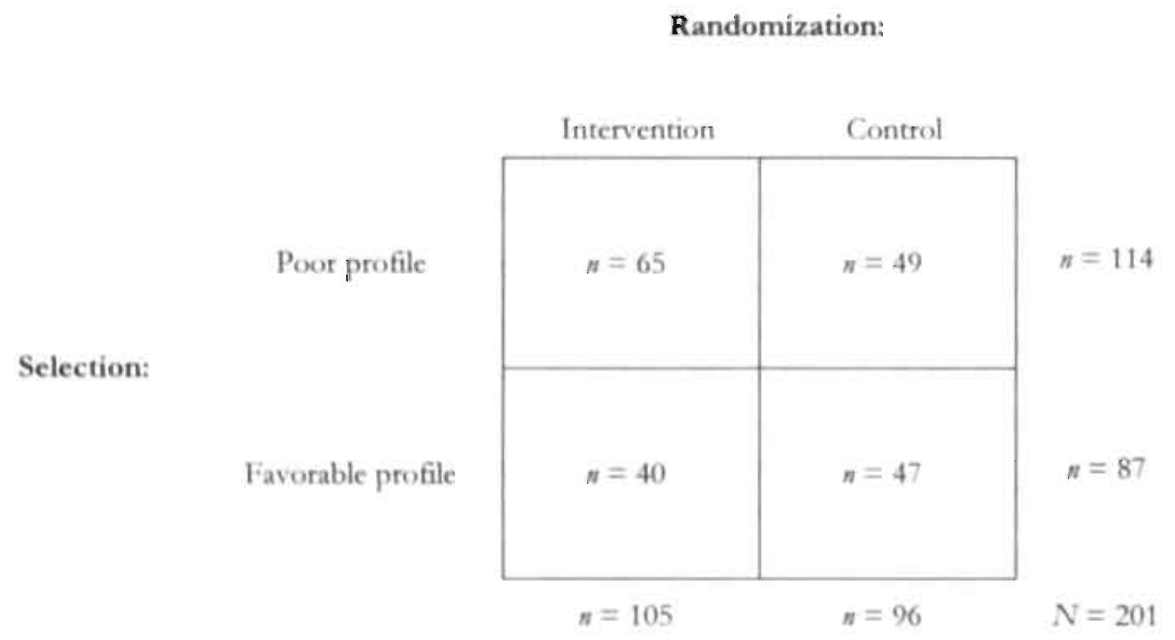


Table 4. Baseline risk factor measures and changes in risk factor measures for poor profile and favorable profile patients in the intervention group and control group

\begin{tabular}{|c|c|c|c|c|c|}
\hline Outcome & Profile & Treatment & $n$ & Baseline $[S D]$ & $\begin{array}{l}\text { Change from } \\
\text { baseline to } \\
\text { 9-month follow- } \\
\text { up [SD] }\end{array}$ \\
\hline \multirow[t]{4}{*}{ Cholesterol } & Poor & I & 49 & $5.2[1.2]$ & $-0.5|1.3|$ \\
\hline & & C & 43 & $5.0[1.0 \mid$ & $+0.1[0.8]$ \\
\hline & Favorable & 1 & 35 & $5.1[1.3 \mid$ & $-0.3|1.2|$ \\
\hline & & C & 41 & $5.2[1.0]$ & $-0.3[1.0]$ \\
\hline \multirow[t]{4}{*}{ Systolic BP } & Poor & I & 51 & $134.2|22.2|$ & $-2.3[18.9]$ \\
\hline & & C & 43 & $128.0[19.6]$ & $+8.5[16.9]$ \\
\hline & Favorable & I & 35 & $126.1[19.8]$ & $+4.7[15.3]$ \\
\hline & & C & 40 & $122.8[18.5]$ & $+7.1[17.9]$ \\
\hline \multirow[t]{4}{*}{ BMI } & Poot & I & 49 & $27.1[3.0]$ & $+0.4[1.5]$ \\
\hline & & C & 45 & $27.2[3.4]$ & $+0.7\lceil 1.5\}$ \\
\hline & Favorable & I & 33 & $25.6[3.3]$ & $+0.2\lceil 1.2\rceil$ \\
\hline & & C & 38 & $25.4[3.2]$ & $-0.0[1.2]$ \\
\hline \multirow[t]{4}{*}{ Smoking } & Poor & 1 & 53 & 10 & +7 \\
\hline & & C & 44 & 5 & +5 \\
\hline & Favorable & 1 & 36 & 3 & +3 \\
\hline & & C & 42 & 2 & +5 \\
\hline \multirow{4}{*}{$\begin{array}{l}\text { Type A } \\
\text { behavior }\end{array}$} & Poor & 1 & 53 & $218.9[55.2]$ & $-68.9|57.9|$ \\
\hline & & C & 44 & $223.0[57.9]$ & $-56.2[67.6 \mid$ \\
\hline & Favorable & I & 36 & $220.8[55.2]$ & $-80.9[62.1]$ \\
\hline & & C & +2 & $197.1[64.4]$ & $-45.9[54.2]$ \\
\hline \multirow{4}{*}{$\begin{array}{l}\text { Vital } \\
\text { Exhaustion }\end{array}$} & Poor & I & 53 & $7.1[5.1]$ & $-1.6|6.4|$ \\
\hline & & C & 43 & $5.4 \cdot[4.7]$ & $+1.0 \mid 4.6]$ \\
\hline & Favorable & I & 36 & $4.2[3.4 \mid$ & $-1.0[5.0]$ \\
\hline & & C & 42 & $3.6[3.3]$ & $-0.9[2.9]$ \\
\hline \multirow[t]{4}{*}{ State anxiety } & Poor & 1 & 47 & $40.8[12.1]$ & $-0.9[10.7]$ \\
\hline & & C & 41 & $40.7[9.8]$ & $-2.0[7.6]$ \\
\hline & Favorable & 1 & 33 & $36.3[10.9]$ & $-1.1[7.2]$ \\
\hline & & C & 40 & $35.0[7.7]$ & $-1.7[7.7]$ \\
\hline \multirow{4}{*}{$\begin{array}{l}\text { Quality of } \\
\text { life }\end{array}$} & Poor & I & 48 & $167.5[32.7]$ & $+15.9[26.4]$ \\
\hline & & C & 42 & $178.4\lceil 25.1]$ & $+4.4[15.0 \mid$ \\
\hline & Favorable & 1 & 35 & $192.3[27.0]$ & $+6.6[23.6]$ \\
\hline & & C & 40 & $191.4[27.6]$ & $+9.4[19.5]$ \\
\hline
\end{tabular}

Note. $\mathrm{I}=$ Intervention group, $\mathrm{C}=$ Control group; $\mathrm{BMI}=$ Body Mass Index in $\mathrm{kg} / \mathrm{m}^{2}$; Total cholesterol in mmol/ $\mathrm{L}$; Systolic blood pressure in $\mathrm{mm} \mathrm{Hg}$. Smoking = number of smoking patients. Range of scores for each measurement: Type $\mathrm{A}$ behavior 2-385, Vital exhaustion 0-23; State Anxiety 20-80; Quality of Life 35-245.

For none of the eight risk factors we studied were the effects of the intervention in the group of poor profile patients significantly different from the effects in the group of favorable profile patients (table 5). The interaction variable Group Allocation (control or intervention) $x$ Profile (poor or favorable profile) was not significant for any of the outcome measures. The intervention effect was only 
larger in poor profile patients compared to favorable profile patients concerning quality of life, with a trend for significance.

Table 5. Results of ANCOVA on 9-month follow-up risk factor levels, analyzing the interaction variable Group Allocation $x$ Profile ${ }^{a}$

\begin{tabular}{|c|c|c|c|c|}
\hline Outcome & $\beta$ & $95 \% \mathrm{CI}(\beta)$ & $p$ & $N$ \\
\hline Cholesterol & -0.2 & $-0.7 / 0.2$ & .34 & 168 \\
\hline Systolic BP & -5.8 & $-14.9 / 3.3$ & .21 & 169 \\
\hline BMI & -0.6 & $-1.4 / 0.2$ & .14 & 164 \\
\hline Type A behavior & 13.1 & $-22.3 / 48.5$ & .47 & 175 \\
\hline Vital Exhaustion & -2.0 & $-4.9 / 0.9$ & .17 & 174 \\
\hline State anxiety & -0.1 & $-5.1 / 4.8$ & .96 & 161 \\
\hline \multirow[t]{2}{*}{ Quality of life } & 10.9 & $-1.2 / 23.0$ & .08 & 165 \\
\hline & OR & $95 \%$ CI (OR) & $p$ & $N$ \\
\hline Smoking & 1.74 & $0.24 / 12.8$ & .59 & 175 \\
\hline \multicolumn{5}{|c|}{ 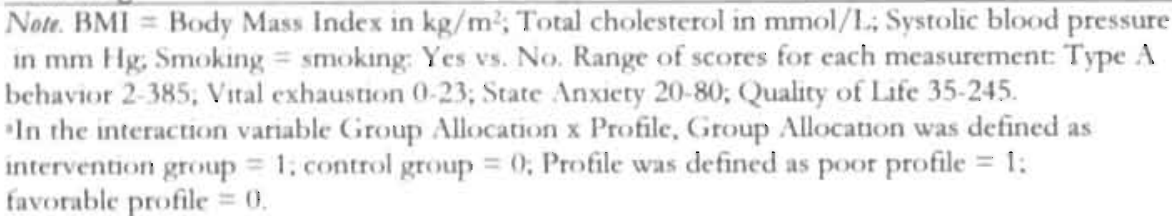 } \\
\hline
\end{tabular}

Post-hoc analyses: Do poor profile patients profit from the intervention as compared with poor profile patients allocated to the control group? And do favorable profile patients profit from the intervention as compared with favorable profile patients in the control group?

A sub analysis with poor profile patients (not in table) showed that the reduction in a number of risk factors was (significantly, or with a trend for significance) larger in patients allocated to the intervention group than in patients in the control group. For poor profile patients, the reduction in total cholesterol level was $0.4 \mathrm{mmol} / \mathrm{L}$. larger in the intervention group than in the control group (ANCOVA, 95\% CI: $0.7 /-0.1, F(1,84)=4.98, p=.03)$. The intervention group also showed a 7.1 $\mathrm{mm} \mathrm{Hg}$ larger reduction in systolic blood pressure compared to the control group $(95 \% \mathrm{Cl}:-13.8 /-0.4, F(1,87)=4.38, p=.04)$, an 0.5 points smaller increase in BMI $\left(95_{0}^{\circ} \mathrm{Cl}:-1.1 / 0.1, F(1,84)=2.88, p=.09\right)$, a reduction in vital exhaustion that was 2.0 points larger $\left(95^{\circ} \circ \mathrm{Cl}:-4.2 / 0.3, F(1,90)=3.10, p=.08\right)$, and an improvement in quality of life that was 8.5 points larger in the intervention group. compared to the control group $\left(95^{\circ}\right.$ C CI: $\left.-0.2 / 17.1, F(1,84)=3.78, p=.06\right)$.

In a sub analysis with favorable profile patients, only type $A$ behavior was reduced to a larger extent in intervention patients compared with control patients, with a difference of 32.9 points $\left(95^{\circ} \circ \mathrm{CI}:-59.5 /-6.2, F(1,72)=6.02, p=.02\right)$. 


\section{DISCUSSION}

The use of screening in cardiac rehabilitation permits professionals to identify patients with a poor profile of risk factors for CAD, who would derive maximal benefit from risk factor modification, while excluding from treatment those who fail to meet the risk cutoff. The results of this study show that it is possible to develop a device to select patients with a poor and a more favorable risk factor profile. The results of this study do not give direct support to the belief that a behavioral intervention is more effective in poor profile patients than in favorable profile patients. For eight CAD risk factors we analyzed whether poor profile patients profited significantly more from the intervention than favorable profile patients did, and apart from a trend for significance in the quality of life analysis, this was not the case.

However, the results of the post-hoc analyses showed that the patients with unfavorable risk factor profiles seem to profit from the intervention, considering the significantly larger reduction these 'poor profile' patients in the intervention group showed in serum total cholesterol level and systolic blood pressure, while BMI, vital exhaustion and quality of life were improved with a trend for significance compared to poor profile patients who only received standard usual care. Patients with relatively favorable risk factor profiles, on the other hand, showed significantly larger reductions in Type A. behavior - and not in any of the other reported. risk factors or psychological variables - if they were allocated to the intervention group compared to 'favorable profile' patients who received usual care. The intervention thus seems to be particularly effective in reducing risk factors and psychological distress in those who need it. For clinicians, referring poor profile patients to a multifactorial intervention seems worth the effort, because on average five out of the eight CAD risk factors we studied will be reduced.

Several reasons may account for the lack of statistical evidence that the intervention is more effective in poor profile patients than in favorable profile patients. First and most importantly, the study size is limited because of the restricted time span due to the grant scheme of the study, resulting in 21 months of patient recruitment. Consequently, we may not have had enough power to detect a significant interaction between intervention effect and risk factor profile. In addition, most patients met our criteria that defined 'poor profile' patients, which led to a relatively small group of 'favorable profile' patients. Second, our study population showed relatively low risk factor levels at entry, which can partly be explained by pharmacotherapy. Fifty-three percent of all patients were already receiving cholesterol-lowering drugs at entry in the study, while $90 \%$ received medication with an effect on blood pressure. The risk factor levels of poor profile patients were relatively low, even though they were higher than the risk factor levels in the favorable profile patients. It is hard to reduce risk factor levels after a multifactorial intervention if the levels are already low. Perhaps statistical effects would have been more convincing if the poor profile patients had been more 
distressed. Third, the statistical null finding might be explained by shortcomings in the screening method, leading to overlap between groups. For one thing, the team making the decision did not have full access to all relevant data. Cholesterol values, for instance, were frequently unknown at the time that the screening questionnaires were evaluated for selection. This is illustrated by the fact that favorable profile patients and poor profile patients had similar cholesterol levels at entry. Since the patients themselves filled out the screening questionnaires, the information that was derived from them was often incomplete and inevitably subjective. Moreover, screening by a team was a more or less subjective process as well. It often involved a process of weighing up the pros and cons for each patient. Is it useful to select CAD patients for a multifactorial behavioral group intervention? Clinicians may feel that it is worth the effort, since patients with a poor CAD risk factor profile showed improvement in. five out of the eight risk factors we studied, whereas patients with a favorable CAD risk factor profile showed improvement in only one. The low risk factor levels displayed by the favorable profile patients in this study cast doubt on the need of additional multifactorial rehabilitation efforts in this group. Although in these patients significant improvement was found in type A behavior, this benefit might disappear with improved screening of type $A$ behavior. Screening of poor profile patients need not be time-consuming and the screening procedure described in this study parallels standard hospital protocol activities. On the other hand, the lack of statistical evidence that screening pays off may argue against the screening and selection of patients with a poor CAD risk factor profile for a multifactorial intervention. Even though the clinician may decide otherwise, we need to study the use of screening for multufactorial interventions in larger study populations, and preferably in more distressed patients. Furthermore, we need to find ways in which the screening of CAD patients for multidisciplinary interventions can be optimized. The question is whether it is possible to select patients properly for an intervention that aims to modify multiple risk factors. We do believe that interventions should be tailored to specific groups of CAD patients. It seems useful to select patients with elevated risk factor levels for an intervention to reduce that particular risk factor. But in order to screen patients for multifactorial rehabilitation programs, the program itself perhaps should be tailored first. Selection of patients for a multifactorial intervention is complicated, and its evaluation possibly even more so. How can we expect that a patient's cholesterol level will drop as a result of the intervention if he or she was selected for this intervention because of - say - his or her hypertension?

In conclusion, although we did not find statistical evidence for the effectivity of pre-selection for multifactorial interventions in the main analyses, subgroup analyses indicate that confining intervention programs to patients with a poor risk factor profile may improve the efficiency of cardiac rehabilitation. Many patients show such low risk factor levels that improvement after an intervention program can hardly be expected. One suggestion for improvement of the current policy may be that a short general multifactorial intervention is offered to groups of patients with a poor risk. factor profile. Individual targets for each patient may then 
be determined, which could be followed by tailored treatments for, e.g., reducing weight or depression. 

CHAPTER 6

GENERAL DISCUSSION 


\section{GENERAL DISCUSSION}

Is the eight-week multiple risk modification group intervention for patients with acute myocardial infarction (AMI) and coronary artery bypass grafting (CABG), which was developed at the University Hospital Mastricht, effective in reducing clinical risk factors and psychological distress? And is it useful to select coronary artery disease (CAD) patients for this intervention? The aim of this thesis was to answer these two major questions. Limitations of the study, which may put the results in perspective, have been discussed in each of the separate previous chapters of this thesis, and will not be repeated. Instead, in this last chapter the results of the study will be briefly reviewed and tentative explanations as well as implications of the findings will be discussed, taking into account the most important restraints of the study. In addition, suggestions will be made for future research.

\section{Effects of the intervention on clinical risk factors and psychological distress}

In a randomized controlled trial, we studied whether patients randomized to the intervention group showed a larger improvement in psychological risk factors for CAD and distress - i.e. type A behavior, vital exhaustion, depression (primary outcome measures in the study), quality of life, and anxiety (secondary outcome measures, appendix chapter 2) - compared to usual care control patients. Furthermore, the effects of the intervention on reducing the clinical factors cholesterol (primary outcome measure), body mass index, blood pressure and smoking (secondary outcome measures - appendix chapter 3 ) were evaluated.

As for emotional distress, the intervention had no overall impact on vital exhaustion, depression (measured by the Beck Depression Inventory), and quality of life. Yet, patients with high vital exhaustion scores at baseline displayed larger reductions of these scores after 9 months if they were allocated to the intervention group as opposed to the control group. The intervention also had a favorable impact on overall type $A$ behavior and more specific on its components hostility and time urgency, both directly after the intervention and after 9 months. On the other hand, state anxiety decreased to a larger extent in the control group than in the intervention group at postintervention. Moreover, unwanted results were found at postintervention concerning the percentage of patients with major depression, favoring the control group. While the percentage of depressed patients in the intervention group increased from $15 \%$ at baseline to $17 \%$ at postintervention, the control group showed a reduction from $9 \%$ tot $5 \%$ at postintervention.

The intervention had no impact on the clinical factors body mass index, blood pressure and smoking. Compared to the control group, patients in the intervention group showed larger reductions in total serum cholesterol level and LDL cholesterol after 9 months, the effect of the intervention being stronger for patients with the highest cholesterol levels at entry. On the other hand, HDL 
cholesterol increases were larger in the control group than in the intervention group.

\section{Is it useful to select coronary artery disease patients for a multifactorial behavioral group intervention?}

AMI patients and CABG patients who were selected through a screening instrument which had been developed at the University Hospital Maastricht, displayed more unfavorable risk factor levels than patients who were not selected through this screening instrument. We studied whether the patients with poorer risk factor levels found more benefit in the intervention than patients with more favorable risk factor levels. Eight risk factors were used as outcome measures, i.e. total. serum cholesterol level, systolic blood pressure, body mass index, smoking, type $A$ behavior, vital exhaustion, state anxiety, and quality of life.

We analyzed for these eight CAD risk factors whether the patients with relatively poor risk factor profiles profited significantly more from the intervention than patients with more favorable risk factor profiles, and this was not the case. In posthoc analyses, however, the group of patients with a more unfavorable risk factor profile showed a (significant or with a trend for significance) favorable impact of the intervention on five out of the eight studied risk factors for CAD. In the group of patients with relatively favorable risk factor levels, on the other hand, the intervention had a favorable impact on only one risk factor.

\section{Explanations for the impact or lack of impact of the intervention}

The results of this trial indicate that the behavioral intervention may result in persistent favorable teductions in some psychological and clinical risk factors. Nevertheless, we may argue that the overall effect of the intervention was modest, since a good few risk factors remained unchanged by the intervention efforts, while other risk factors were increased or favorably altered to a lesser extent than in the control group. Why was the intervention effective in reducing some risk factors while other risk factors stayed behind, or were even altered negatively? Several tentative explanations are discussed below, taking into account strengths and weaknesses of the study.

\section{Characteristics of the intenention}

The intervention was aimed at modifying multiple risk factors, and comprised health education concerning the risk of smoking, elevated blood pressure, obesity, unhealthy dietary habits and elevated cholesterol levels, lack of exercise and type A behavior, while consequences of coronary artery disease such as depression were discussed as well. During the relatively short intervention, explicit attention was paid to the reduction of excessive consumption of dietary fat and its relation to elevated serum cholesterol levels, altering type A behavior, and lack of physical exercise. As much as two sessions were oriented towards altering stress and type $A$ behavior. Following the cognitive social learning model - after the Recurrent Coronary Prevention Project - cognitive, behavioral, environmental and 
physiologic aspects of the type A behavior pattern were treated. Although the sessions had a predominantly health education nature, treatment of type A behavior comprised discussion of basic cognitions frequently recognized in people exhibiting type A behavior, and recommendations of behavioral drills. The favorable impact of the intervention on type $A$ behavior and cholesterol may be explained by these relatively elaborate efforts. Conversely, the risk of smoking or for example - obesity was only briefly discussed, while vital exhaustion, depression, anxiety and quality of life were addressed indirectly or briefly through health education.

The negative findings concerning anxiety and depression are not so easy to account for. Perhaps for some patients the program confronted them with their disease, thereby interfering with their habitual coping strategy of denial. This explanation remains, however, speculative, and deserves future research.

\section{Improved medication adberence}

All patients participating in the study received regular medical check-ups, while pharmacotherapy was initiated if indicated. The overall impact of the intervention on total serum cholesterol level was not associated with altered prescriptions of cholesterol-lowering medication, nor could this unequivocally be explained by altered dietary fat consumption. The explicit attention paid to adverse effects of elevated. blood cholesterol may have encouraged patients in the intervention group to take medication more conscientiously. Unfortunately, we have no means available to study medication compliance in the study population.

\section{Low distress in the study population}

The program had overall impact only on type $A$ behavior and total serum cholesterol levels. Strikingly, for several risk factors intervention effects were more prominent at more unfavorable baseline risk factor levels. The program had a positive impact on total serum cholesterol and LDL cholesterol in patients displaying relatively high baseline levels. Patients with higher baseline levels of vital exhaustion found benefit in the intervention as well, if their reductions in vital exhaustion are compared with patients in the control group. These findings suggest that the program may have a favorable impact in patients who are distressed or who display elevated risk factor levels, thus in those who need it. Scrutinizing the risk factor levels of the patients teaches us that the study group was only moderately distressed at entry of the study. Risk factors were only moderately elevated as well, which may partly pertain to the fact that many patients received. pharmacotherapy before they entered the study. The modest impact of the intervention might partly be explained by these low entry levels. After all, improvement is hardly feasible if patients show only moderately elevated risk. factors to begin with. Future studies should focus on more distressed study populations, such as patients with a score of $\geq 8$ on vital exhaustion, a score of $\geq$ 10 on $\mathrm{BDI}$ depression, or serum total cholesterol levels $>5.0 \mathrm{mmol} / \mathrm{L}$. 


\section{Gender and intenention effects}

Although the number of women was considered too small to permit sub-analyses concerning gender, we may argue that intervention effects for men and women differ based upon previous literature and the limited data we have in the study. A. study on the impact of a psychosocial nursing intervention for patients recovering from myocardial infarction published by Frasure-Smith et al. (Frasure-Smith et al., 1997) found a gender difference in mortality, favoring men. In our own study men seemed to respond to the intervention more favorably than women as well, particularly with regard to anxiety, quality of life and vital exhaustion. Female patients are older, and have been shown to have poorer quality of life after AMI, CABG and PCI compared with male patients, which should be taken into account in the clinical management of these patients, since female patients may need special attention (Westin, Carlsson, Erhardt, Cantor-Graac, \& McNeil, 1999). The recently published Enhancing Recovery in Coronary Heart Disease (ENRICHD) study seems to indicate as well that women tend to be better off in usual care than in a special intervention (Writing Committee for the ENRICHD Investigators, 2003). If so, the question remains why the intervention would be less effective for women than for men.

\section{Usual cane}

We compared the intervention group with a usual care control group. Usual care implies that patients received the medical care they would have received had they not participated in the intervention study. Perhaps very effective usual care in this population reduced the additive value of the intervention. Moreover, the usual care provided to patients that are engaged in research, by definition deviates from regular standard usual care, since patients are interviewed or fill out questionnaires for the purpose of the data gathering process. In itself this may lead to insurmountable dissimilarities with regard to the usual care that is provided under normal, non-research circumstances. It is conceivable that patients in the control group of the study brought about favorable changes in CAD risk factors as a result of the 'extra' usual care per se, thereby reducing the differences between the intervention and control patients.

\section{The validity of self reported outcomes}

The use of self-report measures might have hampered the validity of the results. Among other things, this applies to the results we reported conceming type A behavior. The Structured Clinical Interview was used to measure this behavior pattem, which by itself is no self-report measure since psychomotor characteristics are scored in addition to a self report structured interview. We observed, however, that the answers patients provided in response to the cognitive questions in this interview were not accompanied by likewise psychomotor characteristics. Since these psychomotor scores are what distinguish the clinical interview from selfreport questions, we might question the validity of the results concerning type A behavior. Since we did not use a second, non-self-report measurement device for 
type $\mathrm{A}$ behavior, we have no objective evidence that patients really changed their type A behavior.

\section{Time of follow-up}

For practical reasons, we measured the outcome of the intervention study directly after the intervention, and again after 9 months. Was the time of follow-up long enough to permit us to find relevant effects? A study conducted by Lisspers et al. reported substantial changes in lifestyle behavior as well as profound effects on long-term cardiac events and mortality frequency after a behavioral oriented program. The favorable effects in this study seem to increase after two years of follow-up and become more pronounced over time (Lisspers, 2003).

\section{And what about pre-selection?}

The results of the screening study indicate that the screening instrument, which was developed at the University Hospital Maastricht, was adequate to distinguish AMI or CABG patients with more favorable risk factor levels ('favorable profile') from patients with less favorable risk factor levels ('poor profile'). In the poor profile group, the intervention was effective in (significantly) reducing two out of eight risk factors, while another three risk factors were improved with a trend for significance. In the group of favorable profile patients, only one risk factor was significantly reduced after the intervention. Although this may be a clinically relevant finding, statistically, the patients with more unfavorable risk factor levels did not profit more from the intervention than patients with relatively favorable risk factor levels. Several tentative explanations for this result are discussed below.

\section{Power}

Since the time span of the study was limited due to the grant scheme of the study, the study size is limited. Consequently, we may not have had enough power to detect a significant interaction between intervention effect and risk factor profile. The study is among the first, however, to make an attempt at developing and studying effects of pre-selection for a multifactorial program for CAD patients, and in doing so we hope to give an impulse on subsequent trials in future.

\section{Low distress in the study population}

We discussed before that the study population generally showed low distress and low risk factor levels. Because of this, patients that were selected as patients with poor risk factor profiles did in fact not display very unfavorable risk factor profiles. Because of this, the contrast between 'poor risk factor profile' patients and 'favorable risk factor profile' patients was not large, though it was significant at a statistical level; for instance, the mean score for vital exhaustion was 3.9 in the favorable profile group, compared to 6.8 in the poor profile group. One may wonder whether the differences were relevant at a clinical level. Statistical 
differences in the impact of the intervention between poor profile patients and favorable profile patients may be hard to find because of this relatively small difference between both groups.

\section{Implications for clinical practice and suggestions for further research}

Do the results warrant the routine implementation of the intervention for patients recovering from $\mathrm{AMI}$ or $\mathrm{CABG}$ ? We showed that a relatively short intervention may lead to favorable reductions in type A behavior and total and I.DL cholesterol levels. The clinical relevance of the favorable findings may be illustrated by the fact that favorable effects on total and LDL cholesterol were most profound in patients with elevated levels, thus in those patients who need it. We also found a favorable impact of the intervention. on vital exhaustion in exhausted patients. Notwithstanding the overall impact of the intervention on cholesterol and type A behavior, however, we are inclined to say that the intervention should not be implemented in general health care, because the intervention had unwanted effects with regard to anxiety and depression. At postintervention, state anxiety decreased to a larger extent in the control group than in the intervention group, and while the percentage of patients with major depression in the intervention group (slightly) increased, the control group showed a (small) reduction.

In the previous text we elucidated that much remains obscure concerning the effective or ineffective components of the intervention. Generally, clinical trials studying psychological interventions for CAD patients lack insight of the mechanisms by which programs are effective, and this study is no exception to the rule. For instance, was the impact of the intervention on cholesterol caused by improved compliance to medication? Nowadays, adherence to medicine is probably most important in controlling the levels of blood pressure and cholesterol in patients with CAD. If psychological interventions lead to improved adherence to drug therapy, enormous profits may be realized. Especially since compliance to medication is generally low (Brewer, Chapman, Brownlec, \& Leventhal, 2002). Second, what is the mechanism behind the favorable impact of the intervention on type A behavior? Assuming that the Structured Clinical Interview produced valid data, the question remains whether dysfunctional type $A$ cognitions were altered, or whether the behavior pattern was altered merely at a behavioral level. Third, what is the mechanism behind the unwanted finding conceming depression and anxiety in the study? More generally, how can we accomplish reduced depression and anxiety in coronary patients? Can depression and anxiety in CAD patients be reduced by treating dysfunctional cognitions, i.e. cognitive behavior therapy (CBT)? In the recently published ENRICHD trial, CBT improved depression and social isolation in AMI patients (Writing Committec for the ENRICHD Investigators, 2003). Generally, studies should examine the effective and non-effective elements of psychological interventions.

The possible poorer overall outcome for women in this intervention needs additional studying. Worse outcome for women compared to men in psychological interventions has been reported in other studies (Frasure-Smith et al., 1997). A possible harmful impact of interventions for women underlines the need for 
further research and the inclusion of adequate numbers of women in trials with $\mathrm{AMI}$ and $\mathrm{CABG}$ patients. In general, however, it may be difficult to recruit women for a psychological intervention. In this study, non-response was higher among women than among men, which was reported in previous studies as well (FrasureSmith et al., 1997). Reasons for nonparticipation should be elucidated. In chapter four of this thesis we discussed that more recently published studies seem to be less effective in reducing mortality than older studies, and this was associated with changes in the composition of the study samples. Psychosocial interventions used to be aimed at favorably altering distress and risk factors in men of young age, while nowadays the study populations consist of increasingly larger proportions of older and female patients, partly due to changing inclusion criteria. Future interventions should be modified in line with the altering patient populations at which they are targeted. Intensive research into sex-specific treatments for postAMI and post-CABG women is needed. An as yet uncompleted and unpublished study on the impact of a one-year group stress management intervention specifically for women with coronary heart disease indicates significant reductions in stress and vital exhaustion and improvements in quality of life (Burell, 2003).

Cardiac mortality is frequently used in effect studies of psychosocial interventions to measure the outcome. After all, hard data are needed to demonstrate the value of these programs. The trouble is that the chances of reaching reduced cardiac mortality are diminutive. The rapid development of modern and more complex technology has been accompanied by decreasing mortality and morbidity figures, and these figures will continue to decrease in future. Many studies enroll thousands of patients in order to show positive effects on mortality. Therefore, in more and more trials a combined endpoint is used, like death, reinfaretion, and stroke, Perhaps we should abandon the idea of proving effectivity of psychosocial interventions through figures on mortality impact. From ENRICHD, for example, we cannot (as yet) claim that trearment for depression, even if effective, will improve the chances of survival. Does this mean the intervention is not successful? Reduced depresșion may be worth the effort even without demonstrạble effect on mortality or morbidity. We should consider a variety of psychological outcomes if we measure treatment effects, and examine these from different perspectives.

And what about pre-selection for multifactorial interventions? The results of the effect study show that the impact of the intervention on vital exhaustion and cholesterol level is more profound in patients with more unfavorable levels of these risk factors at entry of the study. The aforementioned ENRICHD study found larger treatment effects for patients with more severe depression than for patients with low depression, which concurs with the results. The results of our screening study, however, indicate that although pre-selection of patients with. relatively poor risk factor profiles may be easy, demonstrating the efficiency of preselection for multifactorial interventions may not be easy. We did not find statistical evidence for the effectivity of pre-selection for multifactorial interventions in the main analyses, but subgroup analyses indicate that confining intervention programs to patients with a poor risk factor profile may improve the efficiency of cardiac rehabilitation. A major problem we encounter is that the 
intervention, for which we are developing tailored selection criteria, is not a tailored program itself. It may be relatively easy to set up selection criteria for a smoking cessation program, but what will be the criteria to select patients for a multifactorial program? Naturally, the most tailored interventions are individual treatments, but these would raise health care costs. A suggestion may be that patients with a poor risk. factor profile are offered a relatively short general intervention comparable to the one presented in this thesis. During the program individually tailored goals can then be set, which may be followed by tailored treatments or even individual treatments.

\section{CONCLUSION}

The short behavioral intervention program evaluated in this study had positive effects on total cholesterol, LDL, type A behavior, and vital exhaustion. It had an unwanted short-term effect on depression, anxiety, and HDL cholesterol. The observation that the number of depressed patients slightly increased in the intervention group raises serious concerns, despite the fact that this observation was not paralleled by the scores on the BDI. Wide implementation of the program requires the inclusion of effective methods to influence depression.

From the modest effects of the intervention on several risk factors we may conclude that each risk factor in a multi-factorial intervention needs its own management. The positive impact of the intervention was largest for the risk factors that received the most elaborate attention. Success of an intervention correlates with the attention given to the separate risk factors. Apparently more is needed for many other risk factors than was provided in the intervention.

The favorable effects of the intervention on cholesterol and exhaustion were particularly found in patients with unfavorable levels at baseline. In general, it was observed that the intervention had little beneficial effects in patients with low risk factor levels. Pre-selection of patients with unfavorable risk factor levels may increase the effectiveness of behavioral intervention programs.

In our opinion, we should not stop short interventions altogether, since despite the fact that long interventions generally show more favorable results, such interventions are not so easy to implement in common hospitals. Moreover, long duration of interventions may lead to low participation, while the infrastructure necessary for implementation may not be feasible and costs will increase. This study showed that several favorable results are achievable in a relatively short period of time. Other risk factors may need more elaborate attention than was offered in the intervention. Moreover, effective components of interventions must be studied in more detail. 



\section{REFERENCES}

1. Aberg, A., Bergstrand, R., Johansson, S., Ulvenstam, G., Vedin, A., Wedel, H., Wilhelmsson, C., \& Wilhelmsen, L. (1983). Cessation of smoking after myocardial infarction. Effects on mortality after 10 years. British Heart Joumal, $49,416-422$.

2. Adult Treatment Panel II (1994). National Cholesterol Education Program. Second report of the expert panel on detection, evaluation, and treatment of high blood cholesterol in adults. Cimzulation, 89, 1333-1363.

3. Allen, J. K. (1996). Coronary risk factor modification in women after coronary artery bypass surgery. Nursing Researth, 45, 260-265.

4. Appels, A. (1990). Mental precursors of myocardial infarction. British Joumal of Psychiatry, 156, 465-471.

5. Appels, A., Jenkins, C. D., \& Rosenman, R. H. (1982). Coronary prone behavior in The Netherlands: a cross-cultural validation study. Journal of Bebavioral Medicine, 5, 83-90.

6. Appels, A., \& Mulder, P. (1989). Excess fatigue as a precursor of myocardial infarction. European Heart Journal, 9, 758-764.

7. Avis, N. E., Smith, K. W., Hambleton, R. K., Feldman, H. A., Selwyn, A., \& Jacobs, A. (1996). Development of the Multidimensional Index of Life Quality. A quality of life measure for cardiovascular disease. Medical Carr, 34, $1102-1120$.

8. Bär, F. W., \& Vonken, H. J. M. (1990). Wat is het nut van hartrevalidatic? [What is the use of cardiac rehabilitation?]. Nederlands Tiydschnft voor de Geneeskende, 143, 107-112.

9. Beaglehole, R. (1990). International trends in coronary heart disease mortality, morbidity, and risk factors. Epidemiologic Reviens, 12, 1-15.

10. Beck, A. T., Rush, A. J., Shaw, B. F., \& Emery, G. (1979). Cognitive theory of depression. New York: Wiley \& Sons.

11. Beck, A. T., Steer, R. A., \& Garbin, M. G. (1988). Psychometric properties of the Beck Depression Inventory: twenty-five years of evaluation. Clinical Psychology Review, 8, 77-100.

12. Bengtsson, K. (1983). Rehabilitation after myocardial infarction: a controlled study. Scandinavian Joumal of Rebabilitation Medicine, 15, 1-9.

13. Berlin, J. A., \& Colditz, G. A. (1990). A meta-analysis of physical activity in the prevention of coronary heart disease. American Josmal of Epidemiology, 132, 612 628.

14. Blankenhorn, D. H., \& Hodis, H. N. (1994). Arterial imaging and atherosclerosis reversal. Arteriosclerosis, Thrombosis, and Vascular Biology, 14, 177. 192.

15. Blumenthal, J. A., \& Emery, C. F. (1988). Rehabilitation of patients following myocardial infarction. Joumal of Consulting and Clinical Psychology, 56, 374-381. 
16. Booth-Kewley, S., \& Friedman, H. S. (1987). Psychological predictors of heart disease: a quantitative review. Psychological Bulletin, 101, 343-362.

17. Bracke, P. E., \& Thoresen, C. E. (1996). Reducing type A behavior patterns: a structured-group approach. In R. Allen. \& S. Scheidt (Eds.), Heart \& Mind. The practice of cardiac psychology (pp. 255-290). Washington, DC, USA: American Psychological Association.

18. Brackett, C. D., \& Powell, L. H. (1988). Psychosocial and physiological predictors of sudden cardiac death after healing of acute myocardial infarction. American Journal of Cardiology, 61, 979-983.

19. Brewer, N. T., Chapman, G. B., Brownlee, S., \& Leventhal, E. A. (2002). Cholesterol control, medication adherence and illness cognition. British Journal of Health Psychology, 7, 433-447.

20. Brezinka, V., \& Kittel, F. (1995). Psychosocial factors of coronary heart disease in women: A review. Social Science and Medicine, 42, 1351-1365.

21. British Association for Cardiac Rehabilitation. (1995). Guidelines for cardiac rebabilitation. Blackwell Science.

22. Brown, B. G., Zhao, X., Sacco, D. E., \& Albers, J. J. (1993). Lipid lowering and plaque regression. New insights into prevention of plaque disruption and clinical events in coronary disease. Circulation, 87, 1781-1791.

23. Burell, G. (1996). Group psychotherapy in Project New Life: treatment of coronary-prone behaviors for patients who have had coronary artery bypass graft surgery. In R. Allen \& S. Scheidt (Eds.), Heart \& Mind. The practice of cardiac psychology (pp, 291-310). Washington, DC, USA: American Psychological Association.

24. Burell, G. (2003). Heart and Lifestyle, New Life, Women's Hearts, and Hope for the Elderly: Behavioural interventions with CAD patients in Sweden. Paper presented at the conference Heart and Mind. The role of stress in coronary artery disease. Maastricht, The Netherlands.

25. Burell, G., Ohman, A., Sundin, O., Ström, G., Ramund, B., Cullhed, I., \& Thoresen, C. E. (1994). Modification of the type A Behavior pattern in postmyocardial infarction patients: A route to cardiac rehabilitation. International Journal of Bebarioral Medicine, 1. 32-54.

26. Burr, M. L., Gilbert, J. F., Holliday, R. M., Elwood, P. C., Fehily, A. M., Rogers, Sweetnam, P.M., \& Deadman, N.M. (1989). Effects of changes in fat, fish, and fiber intakes on death and myocardial reinfarction: diet and reinfarction trial (DART). Lancet, 334, 757-761.

27. Carlsson, R. (1998). Serum cholesterol, lifestyle, working capacity and quality of life in patients with coronary artery disease. Experiences from a hospitalbased secondary prevention programme. Scandinavian Cardiovascular Joumal, 32, 1-20.

28. Carlsson, R., Lindberg, G., Westin, L., \& Israelsson, B. (1997). Influence of coronary nursing management follow up on lifestyle after acute myocardial infarction. Heart, 77, 256-259. 
29. Carney, R. M., Freedland, K. E., Veith, R. C., \& Jaffe, A. S. (1999). Can treating depression reduce mortality after an acute myocardial infarction? Psycbosomatic Medicine, 61, 666-675.

30. Cooper, A., Lloyd, G., Weinman, J., \& Jackson, G. (1999). Why patients do not attend cardiac rehabilitation: role of intentions and illness beliefs. Heart, 82 , 234-236.

31. Corstiens, H. H., Kuijs-Wouters, Y. M. S., Bär, F. W. H. M., \& Falger, P. R. I. (1999). Screening en selectie van patienten voor hartrevalidatie. [Screening and selection of patients for cardiac rehabilitation]. FysioPnaxis(7), 23-26.

32. Daly, L. E., Mulcahy, R., Graham, 1. M., \& Hickey, N. (1983). Long tem effect on mortality of stopping smoking after unstable angina and myocardial infarction. Britisb Medical Joumal, 287, 324-326.

33. DeBusk, R. F., Houston Miller, N., Superko, R., Dennis, C. A., Thomas, R. I.. Lew, H. T., Berger III, W. E., Heller, R. S., Rompf, J., Gee, D., Kraemer, H. C., Bandura, A., Ghandour, G., Clark, M., Shah, R. V., Fisher, 1., \& Barr Taylor, C. (1994). A case-management system for coronary risk factor modification after acute myocardial infarction. Annals of Internal Medicine, 120 , $721-729$.

34. De Lorgeril, M., Renaud, S., Mamelle, N., Salen, P., Martin, I., Monjaud, I, Guidoilet, J., Touboul, P., \& Delaye, J. (1994). Mediterranean alpha-linolenic acid-rich diet in secondary prevention of coronary heart disease. Lancet, 343 , 1454-1459.

35. De Lorgeril, M., Salen, P., Monjaud, I., \& Delaye, I. (1997). The 'diet heart' hypothesis in secondary prevention of coronary heart disease. Luropean Heart Joumal, 18, 13-18.

36. DeBusk, R. F., Houston Miller, N., Superko, R., Dennis, C. A., Thomas, R. I., Lew, H. T., Berger III, W. E., Heller, R. S., Rompf, I., Gee, D., Kraemer, H. C., Bandura, A., Ghandour, G., Clark, M., Shah, R. V., Fisher, I.., \& Barr Taylor, C. (1994). A case-management system for coronary risk factor modification after acute myocardial infarction. Annals of Internal Medicine, 120 , $721-729$.

37. Dornelas, E. A., Sampson, R. A., Gray, J. F., Waters, D., \& Thompson, P. D. (2000). A randomized controlled trial of smoking cessation counseling after myocardial infarction. Preventive Medicine, 30, 261-268.

38. Dracup, K., Meleis, A. I., Clark, S., Clyburn, A., Shields, L., \& Sealey, M. (1984). Group counseling in cardiac rehabilitation: effects on patient compliance. Patient Education and Counseling, 6, 169-177.

39. Dusseldorp, E., Van Elḍeren, T., Maẹ, S., Meulman, J., \& Kraaij, V. (1999). A meta-analysis of psychoeducational programs for coronary heart disease patients. Healtb Psychology, 18, 506-519.

40. Engblom, E., Korpilahti, K., Hämäläinen, H., Puukka, P., \& Rönnemaa, T. (1996). Effects of five years of cardiac rehabilitation after coronary artery bypass grafting on coronary risk factors. American Joumal of Cardiology, 78, 1428 1431 . 
41. Engblom, E., Rönnemaa, T., Hämäläinen, H., Kallio, V., Vänttinen, E., \& Knuts, L. R. (1992). Coronary heart disease risk factors before and after bypass surgery: results of a controlled trial on multifactorial rehabilitation. European Heart Journal, 13, 232-237.

42. Erdman, R. A. M. (1981). Welbevinden bij hartpatiënten. Samenstelling van een Medisch Psychologische Vragenlijst (De MPVH) ter bepaling van het welzijn bij hartpatiënten. [Well-being in heart patients. Construction of a Psychological Questionnaire (The HPPQ) for the determination of well-being in heart patients.J. Unpublished Doctoral dissertation, Erasmus Universiteit Rotterdam, The Netherlands, Lisse: Swets \& Zeitlinger.

43. Erdman, R. A. M., \& Duivenvoorden, H. J. (1983). Psychologic evaluation of a cardiac rehabilitation program: a randomized clinical trial in patients with myocardial infarction. Joumal of Cardiac Rehabilitation, 3, 696-704.

44. Falger, P. R. J. (1989). Life-span development and myocardial infartion: an epidemiological study. Unpublished Doctoral dissertation, Maastricht University, Maastricht, The Netherlands.

45. Falger, P. R. J., Sebregts, E. H. W. J., Van Leuteren, K. S. J., \& Bär, F. W. H. M. $(2000)$. Wederzijdse beinvloeding van de kwaliteit van leven van patiënten en significante anderen na een hartinfarct of een 'coronary artery bypass-graft'operatic. [Impact of depressed mood on mutual quality of life in cardiac patients and partners after a coronary event]. Gedrag e Gezondheid, 28, 274-287.

46. Falger, P. R. J., \& Voorhoeve, P. M. Treatment of vital exhaustion, depression and coronary risk factors through lifestyle changes: a feasibility study in a general health care practice (unpublished).

47. Feunekes, G. I. J., Van Staveren, W. A., De Vries, J. H. M., Burema, J., \& Hautvast, J. G. A. I. (1993). Relative and biomarker-based validity of a foodfrequency questionnaire estimating intake of fats and cholesterol. The American Journal of Clinical Nutrition, 38, 1-8.

48. Feunekes, G. I. J., Van Staveren, W. A., Graveland, F., De Vos, J., \& Burema, J. (1995). Reproducibility of a semiquantitative food frequency questionnaire to assess the intake of fats and cholesterol in The Netherlands. International Journal of Food' Sciences and Nutrition, 46, 117-123.

49. Ficlding, R. (1979). Behavioural treatment in the rehabilitation of myocardial infarction patients. In D. J. Osborne, M. M. Gruneberg, \& J. R. Eiser (Eds.), Researrb in psychology and medicine, Vol. 1: Pbysical aspects, pain, stress, diagnosis and organic damage. (pp. 176-182). London: Academic Press.

50. Fleiss, J. 1. (1994). Measures of effect size for categorical data. In H. Cooper \& L. V. Hedges (Eds.), The bandbook of research syntbesis (pp. 245-260). New York: Russell Sage Foundation.

51. Ford, D. E., Mead, L. A., Chang, P. P., Cooper-Patrick, L., Wang, N., \& Klag, M. J. (1998). Depression is a risk factor for coronary artery disease in men. Anbives of Internal Medicine, 158, 1422-1426.

52. Frasure-Smith, N. Lesperance, F., Prince, R. H., Verrier, P., Garber, R. A., Juneau, M., Wolfson, C., \& Bourassa, M. G. (1997). Randomised trial of 
home-based psychosocial nursing intervention for patients recovering from myocardial infarction. The Lemeet, 350, 473-479.

53. Frasure-Smith, N., Lesperance, F., \& Talajic, M. (1995). Depression and 18 month prognosis after myocardial infarction. Cimwlation, 91, 999-1005.

54. Frasure-Smith, N., \& Prince, R. (1985). The Ischemic Heart Disease Life Stress Monitoring Program: Impact on mortality. Psycbosomatic Medicane, 47, $431-445$.

55. Fridlund, B., Högstedt, B., Lidell, E., \& Larsson, P. A. (1991). Recovery after myocardial infarction: effects of a caring rehabilitation programme. Scandinavian Journal of Caring Sciences, 5, 23-32.

56. Friedman, M., \& Ghandour, G. (1993). Medical diagnosis of type A behavior. American Heart Journal, 126, 607-618.

57. Friedman, M., \& Powell, L. H. (1984). The diagnosis and quantitative assessment of type A behavior: Introduction and description of the Videotaped Structured Interview. Integrative Psychiatry, 2, 123-129.

58. Friedman, M., Powell, L. H., Thoresen, C. E., Ulmer, D., Price, V., Gill, J.J., Thompson, L., Rabin, D.D., Brown, B., Breall, W.S., Levy, R., \& Bourg, E. (1987). Effect of discontinuance of type A behavioral counseling on type A behavior and cardiac recurrence rate of post myocardial infarction patients. American Heart Joumal, 114, 483-490.

59. Friedman, M., Thoresen, C. E., Gill, J. J., Powell, I.H., Ulmer, D., Thompson, L., Price, V.A., Rabin, D.D., Breall, W.S. Dixon, T., Levy, R., \& Bourg, E. (1984). Alteration of type A behavior and reduction in cardiac recurrences in postmyocardial infarction patients. American Heart Journal, 108, 237-248.

60. Friedman, M., Thoresen, C. E., Gill, J. J., Ulmer, D., Powell, L. H., Price, V. A., Brown, B., Thompson, L., Rabin, D. D., Breall, W. S., Bourg, E., 1 evy, R., \& Dixon, T. (1986). Alteration of type A behavior and its effect on cardiac recurrences in post myocardial infarction patients: summary results of the recurrent coronary prevention project. American Heart Journal, 1/2(4), 653-665.

61. Friedman, M., Thoresen, C. E., Gill, J. J., Ulmer, D., Thompson, L., Powell, L., Price, V., Elek, S.R., Rabin, D.D., Breall, W.S., Piaget, G., Dixon, T., Bourg, E., Levy, R.A., \& Tasto, D.L. (1982). Feasibility of altering type A behavior pattern after myocardial infarction. Recurrent Coronary Prevention Project Study: methods, baseline results and preliminary findings. Circulation, 66, 83-92.

62. Giannuzzi, P., Temporelli, P. L., Corrà, U., Gattone, M., Giordano, A., \& Tavazzi, L. (1997). Attenuation of unfavorable remodeling by exercise training in postinfarction patients with left ventricular dysfunction. Results of the Exercise in Left Ventricular Dysfunction (ELVD) trial. Circulation, 96, 17901797 .

63. Gill, J. J., Price, V. A., Friedman, M., Thoresen, C. E., Powell, I.. H., Ulmer, D., Brown, B., \& Drews, F.R.. (1985), Reduction in type A behavior in healthy middle-aged American military officers. American Heart Joumal, 110, 503-514.

64. Gilliss, C. L., Gortner, S. R., Hauck, W. W., Shinn, J. A., Sparacino, P. A., \& Tompkins, C. (1993). A randomized clinical trial of nursing care for recovery from cardiac surgery. Heart e L sung, 22, 125-133. 
65. Gould, A. L., Rossouw, J. E., Santanello, N. C., Heyse, J. F., \& Furberg, C. D. (1995). Cholesterol reduction yields clinical benefit. A new look at old data. Circulation, 91, 2274-2282.

66. Hajek, P., Taylor, T. Z., \& Mills, P. (2002). Brief intervention during hospital admission to help patients to give up smoking after myocardial infarction and bypass surgery: randomised controlled trial. British Medical Journal, 324, 87-90.

67. Hambrecht, R., Niebauer, J., Marburger, C., Grunze, M., Kälberer, B., Hauer, K., Schlierf, G., Kübler, W., \& Schuler, G. (1993). Various intensities of leisure time physical activity in patients with coronary artery disease: effects on cardiorespiratory fitness and progression of coronary atherosclerotic lesions. Journal of American College of Cardiology, 22, 468-477.

68. Haskell, W. L., Alderman, E. L., Fair, J. M., Maron, D. J., Mackey, S. F., Superko, H. R.., Williams, P.T., Johnstone, I.M., Champagne, M.A., Krauss, R.M., \& Farguhar, J.W. (1994). Effects of intensive multiple risk factor reduction on coronary atherosclerosis and clinical cardiac events in men and women with coronary artery disease. The Stanford Coronary Risk Intervention Project (SCRIP). Circulation, 89, 975-990.

69. Havik, O. E., \& Macland, J. G. (1988). Changes in smoking behavior after a myocardial infarction. Health Psychology, 7, 403-420.

70. Hedbäck, B., \& Perk, J. (1987). 5-year results of a comprehensive rehabilitation programme after myocardial infarction. Eumopean Heart Journal, 8, 234-242.

71. Hemingway, H., \& Marmot, M. (1999). Psychological factors in the aetiology and prognosis of coronary heart disease: systematic review of prospective cohort studies. British Medical Journal, 318, 1460-1467.

72. Higgins, H. C., Hayes, R. L., \& McKenna, K. T. (2001). Rehabilitation outcomes following percutaneous coronary interventions (PCI). Patient Education and Counseling, 43, 219-230.

73. Hofman-Bang, C., Lisspers, J., Nordlander, R., Nygren, A., Sundin, Ö., Ohman, A., \& Rydén, I. (1999). Two-year results of a controlled study of residential rehabilitation for patients treated with percutaneous transluminal coronary angioplasty. Eumopean Heart Journal, 20, 1465-1474.

74. Horlick, L., Cameron, R., Firor, W., Bhalerao, U., \& Baltzan, R. (1984). The effects of education and group discussion in the post myocardial infarction patient. Journal of Psychosomatic Research, 28, 485-492.

75. Hunink, M. G. M., Goldman, L., Tosteson, A. N. A., Mittleman, M. A., Goldman, P. A., Williams, L. W., Tsevat, J., \& Weinstein, M. C. (1997). The recent decline in mortality from coronary heart disease, 1980-1990. The effect of secular trends in risk factors and treatment. JAMA, 277, 535-542.

76. Ibrahim, M. A., Feldman, J. G., Sultz, H. A., Staiman, M. G., Young, L. J., \& Dean, D. (1974). Management after myocardial infarction: a. controlled trial of the effect of group psychotherapy. International Joumal of Psychiatry in Medicine, 5 , 253-268.

77. Jacob, R. G., Chesney, M. A., Williams, D. M., Ding, Y., \& Shapiro, A. P. (1991). Relaxation therapy for hypertension: design effects and treatment effects. Annals of Beharioral Medicine, 13, 5-17. 
78. Jacob, R. G., Wing, R., \& Shapiro, A. P. (1987). The behavioral treatment of hypertension: long-term effects. Beharior Tbenapy, 18, 325-352.

79. Jenkins, C. D. (1976). Recent evidence supporting psychologic and social risk factors for coronary disease: Part II. Tbe New England loumal of Medicine, 294, 1033-1038.

80. Jones, D. A., \& West, R. R. (1996). Psychological rehabilitation after myocardial infaretion: multicentre randomised controlled trial. British Medical Joumal, 313, 1517-1521.

81. Kallio, V., Hämäläinen, H., Hakkila, J., \& Luurila, O. J. (1979). Reduction in sudden deaths by a multifactorial intervention programme after acute myocardial infarction. The Lancet, 313, 1091-1094.

82. Karvett, R. L. (1981). Changes in the diet of myocardial infarction patients: effects of nutrition education. Journal of the American Dietetic Asseviation, 79, 660. 667.

83. Killen, J. D., Taylor, C. B., \& Maccoby, N. (1984). Nicotine gum and selfregulation training in smoking relapse prevention. Bebavior Therapy, 15, 234-248.

84. Kop, W. I., Appels, A. P. W. M., Mendes de leon, C. F., de Swart, H. B., \& Bär, F. W. (1994). Vital Exhaustion predicts new cardiac events after successful coronary angioplasty. Psycbosomatic Medicine, 56, 281-287.

85. Kubo, H., Yano, K., Hirai, H., Yabuki, S., \& Machii, K. (1992). Preventive effect of exercise training on recurrent stenosis after percutaneous transluminal coronary angioplasty (PTCA). Japanese Cinculation Joumal, 56, 413 . 421 .

86. Kugler, J., Seelbach, H., \& Krüskemper, G. M. (1994), Effects of rehabilitation exercise programmes on anxiety and depression in coronary patients: a metaanalysis. British Joumal of Clinical Psychology, 33, 401-410.

87. I ane, D., Carroll, D., Ring, C., Beevers, D. G., \& I.ip, G. (2001). Predictors of attendance at cardiac rehabilitation after myocardial infarcrion. Journal of Psycbosomatic Research, 51, 497-501.

88. IaRosa, J. C., Hunninghake, D., Bush, D., Criqui, M. H., Getz, G. S., Gotto, A. M., Grundy, S. M., Rakita, L., Robertson, R. M., Weisfeldt, M. I.., \& Cleeman, J. I. (1990). The cholesterol facts. A summary of the evidence relating dietary fats, serum cholesterol, and coronary heart disease. A joint statement by the American Heart Association and the National Heart, lung, and Blood Institute. Cimulation, 81, 1721-1733.

89. Leren, P. (1970). The Oslo Diet-Heart Study, Eleven-year report. Circulation, 42, 935-942.

90. Lesperance, F., \& Frasure-Smith, N. (2000). Depression in patients with cardiac disease: A practical review. Joumal of Psychosomatic Researth, 48, 379. 391.

91. Levine, G. N., Keaney, J. F., \& Vita, J. A. (1995). Cholesterol reduction in cardiovascular disease. Clinical benefits and possible mechanisms. The New England Journal of Medicine, 332, 512-521.

92. Levine, S. P., Towell, B. L., Suarez, A. M., Knieriem, L. K., Harris, M. M., \& George, J. N. (1985). Platelet activation and secretion associated with emotional stress. Cinculation, 71, 1129-1134. 
93. Linden, B. (1995). Evaluation of a home-based rehabilitation programme for patients recovering from acute myocardial infarction. Intensive and Critical Care Nursing, 11, 10-19.

94. Linden, W. (2000). Psychological treatments in cardiac rehabilitation: review of rationales and outcomes. Journal of Psychosomatic Research, 48, 443-454.

95. Linden, W., \& Chambers, L. (1994). Clinical effectiveness of non-drug treatment for hypertension: a meta-analysis. Annals of Behavioral Medicine, 16, $35-45$.

96. Linden, W., Stossel, C., \& Maurice, J. (1996). Psychosocial interventions for patients with coronary artery disease: A meta-analysis. Archives of Internal Medicine, 156, 745-752.

97. Lisspers, J. (2003). Cardiac rehabilitation and secondary prevention: Lifestyle behaviour change after PCI. It's effects on the progression of atherosclerosis and long term recurrency risk. Paper presented at the conference Heart and Mind. The role of stress in coronary artery disease. Maastricht, The Netherlands.

98. Marchioli, R., Barzi, F., Bomba, E., Chieffo, C., Di Gregorio, D., Di Mascio, R., Franzosi, M. G., Geraci, E., Levantesi, G., Maggioni, A. P., Mantini, L., Marfisi, R. M., Mastrogiuseppe, G., Mininni, N., Nicolosi, G. L., Santini, M., Schweiger, C., Tavazzi, L., Tognoni, G., Tucci, C., \& Valagussa, F. (2002). Early protection against sudden death by n-3 polyunsaturated fatty acids after myocardial infarction. Time-course analysis of the results of the Gruppo Italiano per lo Studio della Sopravvivenza nell'Infarto Miocardico (GISSI)Prevenzione. Cimulation, 105, 1897-1903.

99. Markovitz, J. H., Marthews, K. A., Kiss, J., \& Smitherman, T. C. (1996). Effects of hostility on platelet reactivity to psychological stress in coronary heart disease patients and in healthy controls. Psychosomatic Medicine, 58, 143 149.

100.Marshall, J., Penckofer, S., \& Llewellyn, J. (1986). Structured postoperative teaching and knowledge and compliance of patients who had coronary artery bypass surgery. Heart é Lung, 15, 76-82.

101.Matthews, K. A. (1988). Coronary heart disease and type A behaviors: update on and alternative to the Booth-Kewley and Friedman (1987) quantitative review. Psycbological Bulletin, 104, 373-380.

102.Matthews, K. A., \& Haynes, S. G. (1986). Type A behavior pattern and coronary disease risk. Update and critical evaluation. American Joumal of Epidemiology, 123, 923-960.

103.May, G. S., Eberlein, K. A., Furberg, C. D., Passamani, E. R., \& DeMets, D. L. (1982). Secondary prevention after myocardial infarction: a review of longterm trials. Progress in Cardionascular Diseases, 14, 331-352.

1.04.Mayou, R. A. (1983). A controlled trial of early rehabilitation after myocardial infarction. Journal of Cardiac Rebabilitation, 3, 397-402.

105. McEwen, B. S. (1998). Protective and damaging effects of stress mediators. New England Journal of Medicine, 338, 171-179.

106.McGec, H. M., \& Horgan, J. H. (1992). Cardiac rehabilitation programmes: are women less likely to attend? British Medical Journal, 305, 283-284. 
107.McGovem, P. G., Jacobs, D. R., Shahar, E., Amett, D. K., Folsom, A. R., Blackburn, H., \& Luepker, R.V. (2001). Trends in acute coronary heart disease mortality, morbidity, and medical care from 1985 through 1997: the Minnesota heart survey. Cinsulation, 104(1), 19-24.

108. Meesters, C., \& Appels, A. (1996a). An interview to measure vital exhaustion. I. Development and comparison with the Maastricht Questionnaire. Psybology and Health, 11, 557-571.

109. Meesters, C., \& Appels, A. (1996b). An interview to measure vital exhaustion. II. Reliability and validity of the interview and correlations of vital exhaustion with personality characteristics. Psychology and Health, 11, 573-581.

110. Mendes de Leon, C. F., Powell, L. H., \& Kaplan, B. H. (1991). Change in coronary-prone behaviors in the Recurrent Coronary Prevention Project. Psychosomatic Medicine, 53, 407-419.

111. Miller, T. Q., Smith, T. W., Tumer, C. W., Guijarro, M. I.., \& Hallet, A. J. (1996). A meta-analytic review of research on hostility and physical health. Psycbological Bulletin, 119, 322-348.

112. Miller, T. Q., Tumer, C. W., Tindale, R. S., Posavac, E. I., \& Dugoni, B. L. (1991). Reasons for the trend toward null findings in research on type $A$ behavior. Psychological Bulletin, 110, 469-485.

113. Mitsibounas, D. N., Tsouna-Hadjis, E. D., Rotas, V. R., \& Sideris, D. A. (1992). Effects of group psychosocial intervention on coronary risk factors. Psychotberapy and Psychosomatics, 58, 97-102.

114.Munro, B. H., Creamer, A. M., Haggerty, M. R., \& Cooper, F. S. (1988), Effect of relaxation therapy on post-myocardial infarction patients" rehabilitation. Nursing Research, 37, 231-235.

115. Nederlandse Vereniging voor Cardiologie en de Nederlandse Hartstichting. (1995). Richtlijnen Hartrevalidatie 1995/1996. Den Haag: Nederlandse Hartstichting.

116. Niebauer, J., Hambrecht, R., Velich, T., Hauer, K., Marburger, C., Kalberer, B., Weiss, C., Hodenberg von, E., Schlierf, G., Schuler, G., Zimmermann, R., \& Kübler, W. (1997). Attenuated progression of coronary artery disease after 6 years of multifactorial risk intervention. Role of physical exercise. Cimulation, 96, 2534-2541.

117.NIH Consensus Development Panel on Physical Activity and Cardiovascular Health. (1996). Physical activity and cardiovascular health. IAMA, 276, 241 . 246.

118. Nunes, E. V., Frank, K. A., \& Kornfeld, D. S. (1987). Psychologic treatment for the type A behavior pattern and for coronary heart disease: a meta-analysis of the literature. Psycbosomatic Medicine, 48, 159-173.

119.Oberman, A., Fletcher, G. F. Lee, J., Nanda, N., Fletcher, B. J., Jensen, B., \& Caldwell, E.S. (1995). Efficacy of high-intensity exercise training on left ventricular ejection fraction in men with coronary artery disease (the Training. Level Comparison Study). American Joumal of Cardiology, 76, 643-647. 
120.Ockene, J., Kristeller, J. L., Goldberg, R., Ockene, I., Merriam, P., \& Barrett, S. (1992). Smoking cessation and severity of disease: the Coronary Artery Smoking Intervention Study. Healtb Psychology, $11(2), 119-126$.

121.O'Connor, G. T., Buring, J. E., Yusuf, S., Goldhaber, S. Z., Olmstead, E. M., Paffenbarger, R. S., \& Hennekens, C.H. (1989). An overview of randomized trials of rehabilitation with exercise after myocardial infarction. Circulation, 80 , 234-244.

122. Oldenburg, B., Allan, R., \& Fastier, G. (1989). The role of behavioural and educational interventions in the secondary prevention of coronary heart disease. In P. F. Lovibond \& P. H. Wilson (Eds.), Clinical and abnormal psychology: proceedings of the XXIV International Congress of Psychology of the International Union of Psychological Science (pp. 429-438). Sydney, Australia: International Union of Psychological Science.

123.Oldenburg, B., Perkins, R. J., \& Andrews, G. (1985). Controlled trial of psychological intervention in myocardial infarction. Journal of Consulting and Clinical Psychology, 53, 852-859.

124.Oldridge, N. B., Guyatt, G. H., Fischer, M. E., \& Rimm, A. A. (1988). Cardiac rehabilitation after myocardial infarction. Combined experience of randomized clinical trials. JAMA, 260, 945-950.

125.Ornish, D. (1998). Avoiding revascularization with lifestyle changes: the Multicenter Lifestyle Demonstration Project. American Journal of Cardiology, 82, $72 \mathrm{~T}-6 \mathrm{~T}$.

126.Omish, D., Brown, S. E., Scherwitz, L. W., Billings, J. H., Armstrong, W. T., Ports, T. A., McLanahan, S. M., Kirkceide, R. L., Brand, R. J., \& Gould, L. K. (1990). Can lifestyle changes reverse coronary heart disease? The Lifestyle Heart Trial. The Lancet, 336, 129-133.

127.Omish, D., Scherwitz, L. W., Billings, J. H., Gould, K. L., Merritt, T. A., Sparler, S., Armstrong, W. T., Ports, T. A., Kirkeeide, R. L., Hogeboom, C., \& Brand, R. J. (1998). Intensive lifestyle changes for reversal of coronary heart disease. JAMA, 280, 2001-2007.

128.Omish, D., Scherwitz, L. W., Doody, R. S., Kesten, D., McLanahan, S. M., Brown, S. E., DePuey, E. G., Sonnemaker, R., Haynes, C., Lester, J., McAllister, G. K., Hall, R. J., Burdine, J. A., \& Gotto, A. M. (1983). Effects of stress management training and dietary changes in treating ischemic heart disease. IAMA, 249, 54-59.

129.Perk, J., Hedbäck, B., \& Engvall, J. (1990). Effects of cardiac rehabilitation after coronary artery bypass grafting on readmissions, return to work, and physical fitness. Scandinavian Journal of Social Medicine, 18, 45-51.

130.Pfeffer, M.A., Sacks, F.M., \& Moyé, L.A. (1999). Influence of baseline lipids on effectiveness of pravastatin in the CARE trial. Joumal of the American College of Cardiology, 33, 125-130.

131.Pozen, M. W., Stechmiller, J. A., Harris, W., Smith, S., Fried, D. D., \& Voigt, G. C. (1977). A nurse rehabilitator's impact on patients with myocardial infarction. Medical Care, 15, 830-837. 
132.Ragland, D. R., \& Brand, R. J. (1988). Type A behavior and mortality from coronary heart disease. The New England Journal of Medicine, 318. 65-69.

133.Rahe, R. H., O'Neil, T., Hagan, A., \& Arthur, R. J. (1975). Brief group therapy following myocardial infarction: eighteen-month follow-up of a controlled trial. Intemational Joumal of Pschiatry in Medicine, 6, 349-358.

134. Research Committee. (1965). Low-fat diet in myocardial infarction. The Lanow, 2, 501-504.

135. Research Committee to the Medical Rescarch Council. (1968). Controlled rrial of soya-bean oil in myocardial infarction. The Lancet, 2, 693-699.

136. Rigotri, N. A., McKool, K. M., \& Shiffman, S. (1994). Predictors of smoking cessation after coronary artery bypass graft surgery. Results of a randomized trial with 5-year follow-up. Annals of Intemal Medicine, 120, 287-293.

137.Rigotti, N. A., \& Pasternak, R. C. (1996). Cigarette smoking and coronary heart discase. Risks and management. Candiology Clinics, 14, 51-68.

138. Rigotti, N. A., Thomas, G. S., \& leaf, A. (1983). Fixercise and coronary heart disease. Annual Review of Medicine, 34, 391-412.

139. Rosamond, W. D., Chambless, I. E., Folsom, A. R., Cooper, L. S., Conwill, D. E., Clegg, L., Wang, C.-H., \& Heiss, G. (1998). Trends in the incidence of myocardial infarction and in mortality due to coronary heart discase, 1987 to 1994. Tbe New England Joumal of Medicine, 339, 861-867.

140.Rose, G. A., Thomson, W. B., \& Williams, R. T. (1965). Corn oil in treatment of ischaemic heart disease. British Medical Joumal, I, 1531-1533.

141.Rosenberg, I., Palmer, J. R., \& Shapiro, S. (1990). Decline in the risk of myocardial infarction among women who stop smoking. New Eingland fournal of Medicine, 322, 213-217.

142.Rosenman, R. H., Brand, R. I., Jenkins, C. D., Friedman, M., Straus, R., \& Wurm, M. (1975). Coronary heart disease in the Western Collaborative Group Study. Final follow-up experience of $8 \frac{1}{2}$ years. / AM A, 233, 872-877.

143. Rosenson, R. S., \& Tangney, C. C. (1998). Antiatherothrombotic properties of statins. Implications for cardiovascular event reduction, $I A M A, 279,1643-$ 1650.

144. Rozanski, A., Blumenthal, J. A., \& Kaplan, J. (1999). Impact of psychological factors on the pathogenesis of cardiovascular diesease and implications for therapy. Cinculation, 99, 2192-2217.

145.Sacks, F. M., Pfeffer, M. A., Moye, L. A., Rouleau, J. I.., Rutherford, J. D., Cole, T. G., Brown, L., Warnica, W., Amold, J. M. O., Wun, C., Davis, B. R., \& Braunwald, E. (1996). The effect of pravastatin on coronary events after myocardial infarction in patients with average cholesterol levels. The New England Journal of Medicine, 335, 1001-1009.

146.Salonen, J. T. (1980). Stopping smoking and long-term mortality after acute myocardial infarction. British Heart Journal, 43, 463-469.

147.Salonen, I. T., Hämynen, H., \& Heinonen, O. P. (1985). Impact of a health education program and other factors on stopping smoking after heart attack. Scandinavian Journal of Social Medicine, 13, 103-108. 
148.Sato, I., Nishida, M., Okita, K., Nishijima, H., Kojima, S., Matsumura, N., \& Yasuda, H. (1992). Beneficial effect of stopping smoking on future cardiac events in male smokers with previous myocardial infarction. Japanese Cinculation Joumal, 56, 217-222.

149.Scandinavian Simvastatin Survival Study Group. (1994). Randomised trial of cholesterol lowering in 4444 patients with coronary heart disease: the Scandinavian Simvastatin Survival Study (4S). The Lancet, 344, 1383-1389.

150.Scherwitz, L. W., Brusis, O. A., Kesten, D., Safian, P. A., Hasper, E., Berg, A., \& Siegrist, J. (1995). Lebensstiländerung bei Herzinfarktpatienten im Rahmen der stationaren und ambulanten Rehabilitation. Ergebnisse einer deutschen Pilotstudie. Zeitschrift für Kardiologie, 84, 216-221.

151.Schneider, R. H. (1994). Adrenergic mechanisms in type A behavior. In O. G. Cameron \& e. al (Eds.), Adrenergic dysfunction and psychobiology. (pp. 275-297). Washington, DC: American Psychiatric Press.

152.Schuler, G., Hambrecht, R., Schlierf, G., Niebauer, J., Hauer, K., Neumann, J., Hoberg, E., Drinkmann, A., Bacher, F., Grunze, M., \& Kübler, W. (1992). Regular physical exercise and low-fat diet. Effects on progression of coronary artery disease. Cinculation, 86, 1-11.

153.Sebregts, E. H., Falger, P. R., Bär, F. W., Kester, A. D., \& Appels, A. (2003). Cholesterol changes in coronary patients after a short behavior modification program. International Journal of Behavioral Medicine, 10, 315-330.

154.Sebregts, E. H. W. J. (2003). Secondary prevention in coronary artery disease. Evaluation of a behavorial intervention aimed at the reduction of risk factors in coronary patients. Unpublished doctoral dissertation, Maastricht University, Maastricht, The Netherlands.

155.Sebregts, E. H. W. J., Falger, P. R. J., Appels, A., Kester, A. D. M., \& Bär, F. W. H. M. (2003). Psychological effects of a short behavior modification program in patients with acute myocardial infarction or coronary artery bypass grafting. Submitted for publication.

156.Sebregts, E. H. W. J., Falger, P. R. J., \& Bär, F. W. H. M. (2000). Risk factor modification through nonpharmacological interventions in patients with coronary heart disease. Journal of Psychosomatic Research, 48, 425-441.

157.Shapiro, A. P., \& Jacob, R. G. (1983). Nonpharmacologic approaches to the treatment of hypertension. Annual Revieus in Public Health, 4, 285-310.

158.Shapiro, D., Hui, K. K., Oakley, M. E., Pasic, J., \& Jamner, L. D. (1997). Reduction in drug requirements for hypertension by means of a cognitivebehavioral intervention. American Journal of Hypertension, 10, 9-17.

159.Singh, R. B., Rastogi, S. S., Verma, R., Laxmi, B., Singh, R., Ghosh, S., \& Niaz, M. A. (1992). Randomised controlled trial of cardioprotective diet in patients. with recent acute myocardial infarction: results of one year follow up. British Medical Joumal, 304, 1015-1019.

160.Sivarajan, E. S., Newton, K. M., Almes, M. J., Kempf, T. M., Mansfield, L. W., \& Bruce, R. A. (1983). Limited effects of outpatient teaching and counseling after myocardial infarction: a controlled study. Heart $\stackrel{\sim}{\sim}$ Lang, 12, 65-73. 
161.Smith, T. W. (1.992). Hostility and health: current status of a psychosomatic hypothesis. Health Psycbology, 11, 139-150.

162.Sparrow, D., Dawber, T. R., \& Colton, T. (1978). The influence of cigarette smoking on prognosis after a first myocardial infarction. Joumal of Cbronic Diseases, 31, 425-432.

163.Spielberger, C. D., Gorsuch, R. L., \& Lushene, R. E. (1970). STAI Manual for the State-Trait Anxiety Inventory. Palo Alto: Consulting Psychologists Press, Inc.

164.Stagmo, M., Westin, L., Carlsson, R., \& Israelsson, B. (2001). Long-term effects on cholesterol levels and the urilization of lipid-lowering drugs of a hospital-based programme for secondary prevention of coronary artery disease. Journal of Candionascular Riske, 8, 243-248.

165.Stem, M. I., Gorman, P. A., \& Kaslow, I. (1983). The group counseling v exercise therapy study: a controlled intervention with subjects following myocardial infarction. Archives of Internal Medicine, 143, 1719.1725.

166.Stransky, M., Kälin, M. W., Schwarzenbach, F. H., \& Schär, M. (1986). Intervention study for the risk reduction of coronary infarction. Interwational Joumal of Rebabilitation Reseanch, 9, 381-384.

167.Suinn, R. M., \& Bloom, L. J. (1978). Anxiety management training for pattern A behavior. Joumal of Bebarvoral Medicine, 1, 25-35.

168.Taylor, C. B., Houston-Miller, N., Haskell, W. L., \& DeBusk, R. F. (1988). Smoking cessation after acute myocardial infarction: the effects of exercise training. Addictive Bebarion, 13, 331-335.

169. Taylor, C. B., Houston-Miller, N., Killen, J. D., \& DeBusk, R. F. (1990). Smoking cessation after acute myocardial infarction: effects of a nursemanaged intervention. Annals of Internal Medicine, 113, 118-123.

170.Theorell, T. (1982). Psychosocial intervention as part of the rehabilitation after a myocardial infarction. Intemational Rehabilitation Medicine, 5, 185-188.

171.Thom, T. J. (1989). International mortality from heart disease: rates and trends. Intemational Journal of Epidemiology, 18 (Suppl 1), S20-8.

172.Tunstall-Pedoe, H., Kuulasmaa, K., Mähönen, M., Tolonen, H., Ruokokoski, E., \& Amouyel, P. (1999). Contribution of trends in survival and coronaryevent rates to changes in coronary heart disease mortality: 10-year results from 37 WHO. MONICA Project populations. The Lancet, 353, 1547-1557.

173. Van der Ploeg, H. M., Defares, P. B., \& Spielberger, C. D. (1980). Handleiding bij de Zelf-Beoordelings Vragenlijst, ZBV. [Manual to the Self-Rating Questionnaire, ZBV] . Lisse: Swets \& Zeirlinger.

174.Van Dixhoorn, J. (1997). Gunstige effecten van adem- en ontspanningsinstructie in de hartrevalidatie: een gerandomiseerd follow-up onderzoek gedurende 5 jaar. [Favorable effects of breathing and relaxation instructions in cardiac rehabilitation: A 5-year randomized follow-up study]. Nederlands Tijdschrift noor Geneeskunde, 141, 530-535.

175. Van Dixhoorn, J., Duivenvoorden, H. J., Staal, H. A., \& Pool, J. (1989). Physical training and relaxation therapy in cardiac rehabilitation assessed 
through a composite criterion for training outcome. American Heart Journal, $118,545-552$.

176.Van Elderen, T., \& Dusseldorp, E. (2001). Lifestyle effects of group health education for patients with coronary heart disease. Psychology and Health, 16, 327-341.

177. Van Elderen, T., Maes, S., \& Van den Broek, Y. (1994). Effects of a health education programme with telephone follow-up during cardiac rehabilitation. British Jourial of Clinical Psychology, 33, 367-378.

178. Van Elderen-Van Kemenade, T., Maes, S., \& Van den Broek, Y. (1994). Effects of a health education programme with telephone follow-up during cardiac rehabilitation. British Journal of Clinical Psychology, 33, 367-378.

179.Vlietstra, R. E., Kronmal, R. A., Oberman, A., Frye, R. L., \& Killip, T. (1986). Effect of cigarette smoking on survival of patients with angiographically documented coronary artery disease. $/ A M A, 255,1023-1027$.

180.Wageningen University. (1993). VET Expres. De voedselfrequentievragenlijst naar de vetconsumptie. [A food frequency questionnaire estimating the intake of fats|. Wageningen, The Netherlands: Wageningen University.

181. Wallner, S., Watzinger, N., Lindschinger, M., Smolle, K. H., Toplak, H., Eber, B., Dittrich, P., Elmadfa, I., Klein, W., Krejs, G. J., \& Wascher, T. C. (1999). Effects of intensified lifestyle modification on the need for further revascularization after coronary angioplasty. European Joumal of Clinical Imestigation, 29, 372-379.

182. Watts, G. F., Lewis, B., Brunt, J. N. H., Lewis, E. S., Coltart, D. J., Smith, L. D. R., Mann, I. 1., \& Swan, A. V. (1992). Effects on coronary artery disease of lipid-lowering diet, or diet plus cholestyramine, in the St 'Thomas' Atherosclerosis Regression Study (STARS). The Lancel, 339, 563-569.

183. West of Scotland Coronary Prevention Study Group. (1998). Influence of pravastatin and plasma lipids on clinical events in the West of Scotland Coronary Prevention Study (WOSCOPS). Circulation, 97, 1440-1445.

184. Westin, L., Carlsson, R., Erhardt, L., Cantor-Graae, E., \& McNeil, T. (1999). Differences in quality of life in men and women with ischemic heart disease. A prospective controlled study. Scandinavian Cardionascular Joumal, 33(3), 160-165.

185. Widdershoven, J., \& De Vreede-Swagemakers, J. (1997). Acute Comnary Syndromes in the Maastricht Area. Unpublished Doctoral dissertation, Maastricht University, Maastricht, The Netherlands.

186. Wilhelmsson, C., Vedin, I. A., Elmfeldt, D., Tibblin, G., \& Wilhelmsen, I. (1975). Smoking and myocardial infarction. Lancet, 1, 415-419.

187. Williams, R. B., Suarez, E. C., Kuhn, C. M., Zimmerman, E. A., \& Schanberg, S. M. (1991). Biobehavioral basis of coronary-prone behavior in middle-aged men. Part 1: Evidence for chronic SNS activation in Type As. Psychosomatic Medicine, 53, 517-527.

188. Woodhill, I. M., Palmer, A. I., Leelarthaepin, B., McGilchrist, C., \& Blacket, R. B. (1967). Low fat, low cholesterol diet in secondary prevention of coronary heart disease. Adnances in Experimental Medicine and Biology, 317-330. 
189. Writing Committee for the ENRICHD Investigators. (2003). Effects of treating depression and low perceived social support on clinical events after myocardial infarction. The Enhancing Recovery in Coronary Heart Disease Patients (ENRICHD) Randomized Trial. JALLA, 289, 3106-3116.

190.Wulsin, L. R., Vaillant, G. E., \& Wells, V. E. (1999). A systematic review of the mortality of depression. Pychosomatic Mediane, 61, 6-17.

191.Yen, L., Patrick, W. K. \& Chie, W. (1996). Comparison of relaxation techniques, routine blood pressure measurements, and self-learning packages in hypertension control. Preventive Mediane, 25, 339-345.

192. Young, D. T., Kottke, T. E., McCall, M. M., \& Blume, D. (1982). A prospective controlled study of in-hospital myocardial infarction rehabilitation. Joumal of Candiac Rebabilitation, 2, 32-40. 



\section{SUMMARY}

Coronary artery disease (CAD) is the leading cause of death in most developed countries. Although mortality rates from CAD are declining as a consequence of prevention efforts and improved medical treatment after acure myocardial infarction, the number of hospitalizations from CAD is growing, since the patient's life span is lengthened. Patients may, however, suffer more often from heart failure when older. Secondary prevention of CAD is of major importance since many patients survive their myocardial infarction. One approach to the secondary prevention of CAD is by means of interventions aimed at behavior modification. but successful interventions are usually lengthy and thereby expensive programs, which may not be easily implemented in general health care. This thesis focuses primarily on the effectiveness of a relatively short intervention that was based on promising and successful - but length - trials of the past decades.

In chapter 1 of this thesis, a review is described concerning the effects of nonpharmacological interventions on risk factor modification in patients with coronary artery disease. A MEDLINE and PSYCHlit computer search was performed to identify studies on non-pharmacological interventions aimed at modification of smoking, serum cholesterol, physical exercise, type A behavior, hypertension or body weight. In order to study the effects of risk factor modification by means of non-pharmacological interventions in patients suffering from CAD, we scrutinized every risk factor separately. The risk of each of these factors on C.AD is outlined before relevant intervention studies are presented. Each factor was amenable to modification through interventions aimed at behavior change. Interventions aimed at healthy lifestyles may stimulate smoking cessation rates, reduce elevated serum total and L.DI. concentrations and favorably modify type $A$ behavior in C.AD patients. Moreover, reduction of coronary atherosclerosis has been reported after intensive lifestyle and exercise interventions, whereas exercise and type $\mathrm{A}$ behavior interventions may also lead to reduced CAD morbidity and mortality. As for hypertension and obesity, studies aimed at secondary prevention are lacking. The chapter concludes with an outline of limitations of the study and suggestions for improvement.

The contents of the intervention that is evaluated in this thesis were primarily based on the methods of three major long-term trials, the Recurrent Coronary Prevention Project (Bracke \& Thoresen, 1996), Project New I.jfe (Burell, 1996), and the I ifestyle Heart Trial (Omish et al., 1990). During the period of February 1996 until November 1997, consecutive patients admitted to the University Hospital Maastricht were identified as eligible for the study. Patients included were less than 70 years of age, had a confirmed diagnosis of acute myocardial infarction, coronary artery bypass grafting, or both, and were able to participate in the regular groupwise physiotherapy exercise program at the hospital. Patients were randomly assigned to either the intervention or a usual care control group. Both groups received usual medical care, while patients that were assigned to the intervention group enrolled into a multidisciplinary multiple risk modification program for 
prevention of CAD, Measurements were performed or collected (1) at baseline, before the beginning of the intervention, which was approximately four weeks after hospital discharge; (2) eight weeks later, immediately after the intervention (postintervention); (3) nine months after termination of the intervention, which was approximately one year after discharge. Two hundred-and-four patients gave signed informed consent (106. were randomized to intervention group, 98 to control group), which was $67 \%$ of all eligible patients. Of these patients, 20 dropped out, reducing the total number of patients in the study to 184 .

Effects of the intervention on outcome measures were assessed by means of analyses of covariance, using at least baseline scores as a covariate. After the main analyses, an interaction variable was included in the analyses to check whether intervention effects were different for patients with high or low baseline scores.

The effects of the intervention on primary psychological outcome measures type $A$ behavior, vital exhaustion and depression are described in chapter 2, whereas secondary outcome measures are added to the chapter as an appendix. Type A behavior was measured by a Dutch version of the Videotaped Structured Interview, Vital Exhaustion by the Maastricht Interview for Vital Exhaustion, and depressed mood state was measured by the Beck Depression Inventory (BDI) and the structured interview according to the Diagnostic and Statistical Manual of Mental. Disorders classification of Major Depressive Disorder (SCID). At baseline, patients randomized to the intervention group had higher scores on vital exhaustion and BDI depression than patients in the control group, whereas other variables were comparable in both groups. The results showed that the reduction. in overall type A behavior at 9-month follow-up was significantly larger in the intervention group than in the control group. Both hostility and time urgency, two major components of type A behavior, were reduced to a larger extent in the intervention group than in the control group at postintervention and at 9-month follow-up. No significant intervention effects were reported on vital exhaustion, although a significant interaction and subsequent sub-analysis of patients with relatively high baseline scores showed that the intervention was effective in. reducing vital exhaustion in patients with relatively high baseline scores. The intervention, did not reduce depression as measured by the BDI. At postintervention, major depression, assessed by the SCID, was significantly less prevalent in the control group. With a trend for significance, the control group showed a larger reduction of state anxiety at postintervention. The intervention did not have an effect on quality of life. The results thus show that several psychological risk factors can be reduced in coronary patients through a relatively short intervention aimed at behavior change and risk reduction. The positive implication of these results is, however, restrained by the unwanted findings concerning anxiety and major depression. Results are compared with relevant trials from the past, and are discussed considering the contents of the program and limitations of the study.

Chapter 3 describes the effects of the intervention on the primary outcome measure serum cholesterol. Clinical data were collected by a cardiologist who was blinded to the patients' group allocation. Dietary fat consumption was measured 
through a self-report questionnaire, the 'VET-expres'. Baseline demographic and clinical characteristics of the intervention and control group did not differ. At 9. month follow-up, total cholesterol was significantly reduced in the intervention group compared to the control group. This effect was especially strong in patients with high baseline total cholesterol levels. LDL cholesterol was also reduced to a larger extent in the intervention group as compared to the control group. Again, the effects were strongest for patients with high baseline 1.DI. cholesterol. During the 9-month period of follow-up, the intervention group had a larger increase of prescribed dose of statins than the control group, and correcting the analyses for this difference weakened the favorable effects of the intervention on LDI. and total cholesterol, although the favorable effects on total cholesterol still showed a trend for significance. In patients with high baseline cholesterol levels, the intervention had a beneficial effect on total and LDI cholesterol irrespective of the taking of statins. Although dietary fat consumption declined to a larger extent in the intervention group than in the control group (with a trend for significance), the change in dietary fat consumption was not found to contribute to the reduction in serum cholesterol. Remarkably, reduced fat consumption during follow-up was associated with reduced cholesterol levels only in patients with low baseline fat consumption. An unexpected finding was a lower increase in $\mathrm{HDL}$ cholesterol in the intervention group than in the control group. Results are discussed from the perspective of the reliability of the dietary questionnaire and regression to the mean. Adherence to medication regimen is considered as a mediating factor. It is concluded that the positive indications of the findings are restrained by an unexpected finding that the increase in HDI. cholesterol was largest in the control group.

The intervention had no impact on the secondary outcome measures smoking, Body Mass Index and blood pressure. During the 9 months of follow-up, the intervention group had a larger decrease of prescribed dosage of AC.E inhibitors and Calcium antagonists. If the analyses were corrected for change in prescribed dosage from baseline to follow-up, the effects of the intervention on systolic blood pressure reached a trend for significance in favor of patients in the intervention.

Recurrent morbidity rates in this study were low during follow-up, and none of the patients died of a cardiovascular cause. Generally, many studies suffer from a lack. of power to investigate effects on mortality, and studies are often combined in a meta-analysis in order to increase power. In chapter 4 , the influence of secular trends on the effects of psychoeducational intervention studies are investigated. The following questions are studied: 1) Are the reported effects of psychoeducational interventions on cardiac mortality rates and recurrent infarctioni rates stable over tume? 2) If not, may this change be explaned by a) changing cardiovascular mortality rates and changing rates of recurrent myocardial infarction, b) altering characteristics of study populations in the course of time? The data of the most recently published meta-analysis concerning the effects of psychoeducational programs on cardiac mortality and morbidity rates in (AD) parients were used (Dusseldorp et al., 1999), which included only relatively highquality studies. We additionally performed a MEDI.INE and PSYC.INFO 
computer search to identify studies published after 1998. Forty-five studies concerning the effects of psychoeducational programs for CAD patients were included in the study. In the 11 studies reporting 12-month recurrent myocardial infarction rates, the year of publication of the studies does not correlate with the effect of the interventions on the rate of recurrent infarctions. In the 8 studies reporting 12-month cardiac mortality rates, the year of publication of the studies correlates negatively with the odd ratio's of cardiac mortality rates; thus, older studies reported larger favorable effects of interventions on cardiac mortality rates than more recently published studies. Although mortality rates seem to decline over rime in these studies, the decline is not significant, and the lower mortality rates in the studies are not associated with the smaller effects of the interventions on mortality. The smaller effects on mortality are, however, associated with mean age of the study population, which is older in more recent studies. We studied changes in population characteristics in 45 studies, and it was found that CAD patients included in more recently published studies are older than patients in older studies. Furthermore, more recently published studies are characterized by a higher percentage of women compared to older studies. The changing population characteristics are associated with changing inclusion criteria in the studies. The results show that meta-analytic studies are losing their external validity. We discuss that the changing patient characteristics may affect the scope of the interventions. Interventions used to be designed for and directed at the improvement of health status in young men, whereas studies include increasingly older and more often female patients.

Professionals stress the need for tailored programming in cardiac rehabilitation, but studies that evaluate the merits thereof are lacking. The primary aims of chapter 5 were to examine the quality of a screening device that had been developed to select patients for a multifactorial intervention, and to determine whether selected patients profited more from a multifactorial intervention than patients, who did not meet the selection criteria. All 204 patients included in the effect study were screened in order to select patients with an unfavorable risk factor profile for coronary artery disease. The hypothesis that patients with a poor profile would profit more from the intervention than patients with a favorable profile was tested by including an interaction variable in the analyses that were used to compute the effects of the intervention on clinical and psychological risk factors: Selected patients showed more unfavorable risk factor levels than unselected patients. The interaction variable was not significant for any of the eight studied risk factors, indicating that the effects of the intervention did not differ between those with or without a poor risk factor profile. A post-hoc sub analysis with poor profile patients showed that for this group, the intervention was (significantly) effective in reducing cholesterol and systolic blood pressure levels, while favorable effects on Body Mass Index, vital exhaustion and quality of life reached a trend for significance. A sub analysis with favorable profile patients showed that in this group the intervention was significantly effective in reducing one risk factor, i.e. type A behavior. The use of pre-selection for multifactorial interventions should be studied in larger, and preferably more distressed, study 
populations. In sum, although we did not find statistical evidence for the effectivity of pre-selection for multifactorial interventions in the main analyses, subgroup analyses indicate that confining intervention programs to patients with a poor risk factor profile may improve the efficiency of cardiac rehabilitation.

In the general discussion the results of the study are briefly reviewed and tentative explanations for the impact (or lack thereof) of the intervention and pre-selection are discussed, taking into account the most important restraints of the study. Implications of the findings for clinical practice are discussed and suggestions are made for future research. Among other things, it is suggested that future studies should examine the effective and non-effective components of psychological interventions in more detail. 



\section{SAMENVATTING}

Hart- en vaatziekten zijn de belangrijkste doodsoorzaak in Nederland, zoals in de meeste ontwikkelde landen het geval is. Met name coronarialijen is verantwoordelijk voor een groot sterftepercentage. Hocwel de sterfte aan ischemische hartziekten dalende is ten gevolge van inspanningen met betrekking tot preventie en verbeterde behandeling van het acute myocard infaret, neemt het aantal ziekenhuisopnamen ten gevolge van ischemische hartziekten nog steeds gestaag toe. Dit is mede te danken aan de vergrijzing en aan het feit dat patiènten door verbeterde medische kennis langer in leven worden gehouden. Het aantal patienten met hartfalen ten gevolge van een eerder doorgemaakt infaret neemt daarmee echter toe. Secundaire preventie van coronarialijden is van groot belang, aangezien veel patiënten tegenwoordig hun myocard infarct overleven. Een van de benaderingen voor secundaire preventie van coronarialijden bestaat uit het aanbieden van interventies die gericht zijn op gedragsverandering. Interventies die succes boeken in het verminderen van risicofactoren en psychosociale problematiek zijn echter vaak langdurig en dientengevolge weinig motiverend voor de patiènt, duur, en onpraktisch, waardoor implementatic in de algemene gezondheidszorg wordt bemoeilijt. In dit proefschrift ligt de nadruk op de effectiviteit van een relatief korte interventie waarvan de inhoud is gebaseerd op veelbelovende en effectiev - maar langdurig - studies van de afgelopen decennia.

In hoofdstuk 1 van dit proefschrift wordt een overzicht gegeven van studies naar de effectiviteit van niet-farmacologische interventies op verandering van risicofactoren bij patiënten met coronarialijden. De literatuurbestanden MEDLINE en PSYCHlit werden systematisch doorzocht om studies te identificeren betreffende niet-farmacologische interventies gericht op verandering van rookgedrag, cholesterolgehalte, lichaamsbeweging, type A gedrag, hypertensie of lichaamsgewicht. Om de effecten van niet-farmacologische interventies op verandering van risicofactoren bij patiènten met coronanalijden te bestuderen, werd elke risicofactor apart in detail onderzocht. Alvorens de relevante interventiestudies worden beschreven, wordt eerst het risico geschetst dat elk van deze factoren heeft op coronarialijden. Elke risicofactor bleck vervolgens ontvankelijk te zijn voor verandering door middel van interventies. die zijn gericht op gedragsverandering. Interventies gericht op een gezonde leefstijl kunnen het stoppen met roken bevorderen, verhoogde concentraties totaal- en I.DI. cholesterol in het bloed verlagen, en type A gedrag bij patiènten met coronarialijden ten gunste beinvloeden. Bovendien wordt er melding gemaakt van een gunstige beinvloeding van het atherosclerotische proces na interventies gericht op intensieve veranderingen van leefstijl en lichaamsbeweging. Interventies gericht op bevordering van lichaamsbeweging en verandering van type A gedrag zouden ook kunnen leiden tot afname van morbiditeit en mortaliteit ten gevolge van ischemische hartziekten. Studies gericht op secundaire preventie van hypertensie en overgewicht bij deze patientengroep werden niet gevonden. Het hoofdstuk 
wordt afgesloten met een bespreking van beperkingen van de studie, alsmede suggesties voor verbetering.

De interventie die in dit proefschrift wordt geëvalueerd is voornamelijk gebaseerd op de methoden van drie belangrijke langdurige studies, nl. het 'Recurrent Coronary Prevention Project' (Bracke \& 'Thoresen, 1996), 'Project New Life' (Burell, 1996), en de 'Lifestyle Heart Trial' (Omish et al,, 1990). Van alle achtereenvolgende patiënten die gedurende de periode tussen februari 1996 en. november 1997 werden opgenomen in het Academisch Ziekenhuis Maastricht (AZM), werd beoordeeld of zij in aanmerking kwamen voor deelname aan de studie. Patiënten werden geincludeerd als ze jonger waren dan 70 , een diagnose hadden van een acuut myocard infarct en / of een coronaire bypass operatie hadden ondergaan, en in staat waren deel te nemen aan de reguliere groepsfysiotherapie in het AZM. Patienten werden aselect toegewezen aan ofwel de interventie, ofwel een controlegroep. Beide groepen ontvingen standaardzorg, terwiil patiènten die waren toegewezen aan de interventiegroep aanvullend een. multidisciplinair programma kregen aangeboden ter preventie van coronarialijden, gericht op verandering van risicofactoren. De metingen werden uitgevoerd. 1) voor aanvang van het programma, ongeveer vier weken na ontslag uit het ziekenhuis (voormeting); 2) acht weken later, onmiddellijk na de interventie; 3) negen maanden na beeindiging van de interventie, hetgeen ongeveer een jaar na ontslag was. Tweehonderdvier patiènten gaven toestemming (waarvan er 106 aselect werden toegewezen aan de interventicgroep en 98 aan de controlegroep), hetgeen $67 \%$ van alle in aanmerking komende patienten was. Van deze patienten trokken. 20 patienten zich terug, waardoor het totale aantal patienten in de studie daalde naar 184.

Effecten van de interventie op de uitkomstmaten werden berekend door middel van covariantic analyses, waarbij in elk geval de voormeting werd gebruikt als covariaat. $\mathrm{Na}$ de hoofdanalyses werd een interactievariabele tocgevoegd om te bekijken of er verschillen waren in effecten tussen patiënten met hoge of lage scores op de voormeting.

De effecten van de interventie op de primaire psychologische uitkomstmaten type A gedrag, vitale uitputting en depressie worden beschreven in hoofdstuk 2, terwijl de secundaire uitkomstmaten aan het hoofdstuk zijn toegevoegd als bịlage. Type $\mathrm{A}$ gedrag werd gemeten d.m.v. een. Nederlandse versie van het 'Videotaped Structured Interview', Vitale Uitputting door middel van het Maastrichtse Interview voor Vitale Uitputting, en depressieve stemming werd gemeten met de Beck Depression Inventory (BDI) en het gestructureerde interview volgens de DSM-III-R classificatie van een Depressieve Stoornis (SCID). Bij de voormeting hadden patienten die aselect waren toegewezen aan de interventiegroep hogere scores dan patienten in de controlegroep met betrekking tot vitale uitputting en depressic zoals gemeten door de BDI, terwijl de andere variabelen in beide groepen vergelijkbaar waren. De reductie in de totaalscore voor type A gedrag was na 9 maanden in de interventiegroep significant groter dan in de controlegroep. Zowel vijandigheid als tijdsdruk, twee hoofdcomponenten van type A gedrag, daalden sterker in de interventiegroep dan in de controle groep; zowel bij de eerste 
nameting als na 9 maanden. Er werden geen significante interventie effecten gevonden met betrekking tot vitale uitputting. Wel werd door een significante interactie en een daarop volgende sub-analyse van patienten met relatief hoge uitputtingsscores op de voormenng aangetoond, dat de interventie leidde tot een verlaging van vitale uitputting bij patienten met relatief hoge scores op de voormeting. Er werden geen effecten gevonden met betrekking tot depressie zoals gemeten door de BDI, maar het percentage patienten met een depressieve stoornis zoals gemeten door het SCID interview liet op de eerste nameting een voordeel zien voor de controlegroep ten opzichte van de interventiegroep. Met een trend voor significantie was direct na de interventie in de controlegroep ook een grotere afname te zien in de secundaire uitkomstmaat toestandsangst, terwijl er geen effecten werden gevonden met betrekking tot kwaliteit van leven. De resultaten van de studie laten dus zien dat verschillende psychologische risicofactoren bij patienten met coronarialijen kunnen worden verlaagd door middel van een relatief korte interventie gericht op gedragsverandering en risicoverlaging. De positieve implicatie van deze resultaten wordt echter beperkt door de negatieve bevindingen met betrekking tot angst en depressieve stoornis. De resultaten worden vergeleken met relevante studies uit het verleden, en worden bediscussieerd in het licht van de inhoud van het programma en de beperkingen van de studie.

In hoofdstuk 3 worden de effecten van de interventie beschreven op de primaire utkomstmaat cholesterol. Klinische data zijn verzameld door een cardioloog die was geblindecrd m.b.t. de groepstoewijzing van de patienten. Vetconsumptic werd gemeten door middel van een zelfrapportage vragenlijst, de 'VET-expres'. Eir waren op de voormeting geen verschillen tussen de interventiegroep en controlegroep met betrekking tot demografische en klinische kenmerken. Na9) maanden was het totaal cholesterol in de interventiegroep significant gedaald vergeleken met de controlegroep. Dit effect was met name aanwezig bij patienten met een hoog totaal cholesterolgehalte op de voormeting. De daling in I.DI. cholesterol was ook groter in de interventiegroep dan in de controlegroep, en ook deze effecten waren het sterkst bij patienten met hoge L.DI-waarden op de voormeting. Gedurende de 9 maanden follow-up was er sprake van een grotere verhoging van dosering van statines in de interventiegroep dan in de controlegroep. Als de analyses werden gecorrigeerd voor dit verschil werden de gunstige effecten van de interventie op LDL en totaal cholesterol enigszins afgezwakt, hoewel de gunstige effecten op totaal cholesterol nog aanwezig waren met een trend voor significantie. Bij patiẹten met hoge cholesterolwaarden op de voormeting had de interventie een gunstig effect op total en LDL cholesterol ongeacht dosering van statines. Hoewel vetconsumptie in de interventiegroep een grotere daling liet zien dan in de controlegroep (met een trend voor significantie), was de verandering in vetconsumptie niet gerelateerd aan de reductie van het cholesterol. Merkwaardig genoeg was slechts bij patiènten met een lage vetconsumptie op de voormeting afgenomen vetconsumptie geassociecrd met afgenomen cholesterolniveaus. Een onverwacht resultaat was dat de stujging van HDL cholesterol groter was in de controlegroep dan in de interventiegroep. De 
resultaten worden besproken in het licht van de betrouwbaarheid van de voedselvragenlijst en regressie naar het gemiddelde. Therapietrouw met betrekking tot medicatie wordt besproken als mogelijke mediërende factor. Er wordt geconcludeerd dat de positieve implicaties van de bevindingen worden beperkt door de onverwachte bevinding dat de toename in HDL cholesterol groter was in de controlegroep dan in de interventiegroep.

De interventic had geen invloed op de secundaire uitkomstmaten roken, queteletindex en bloeddruk. Gedurende de 9 maanden van follow-up was er sprake van een grotere afname in voorgeschreven dosering ACE remmers en Calcium antagonisten in de interventiegroep vergeleken met de controlegroep. Als in de analyses werd gecontroleerd voor verandering in voorgeschreven dosering van voormeting tot follow-up, bereikten de effecten van de interventie op systolische bloeddruk een trend van significantie ten voordele van patienten in de interventiegroep.

In de studie was de morbiditeit gedurende follow-up laag, en niet één. patiënt overleed ten gevolge van een cardiovasculaire oorzaak. Veel studies hebben onvoldoende power om effecten van interventies op mortaliteit te onderzoeken, met als gevolg dat studies vaak worden samengevoegd in een meta-analyse zodat de power om bepaalde effecten te berekenen toeneemt. In hoofdstuk 4 wordt de invloed van trends bestudeerd die zich de afgelopen jaren in studies hebben voltrokken op de effecten van psycho-educatieve interventiestudies. De volgende vraagstellingen worden bestudeerd: 1) Zijn de gerapporteerde effecten van psychoeducatic interventies op cardiovasculaire mortaliteit en het aantal terugkerende infarcten stabiel gebleven de afgelopen decennia? 2) Indien dit niet het geval is, kan deze verantering dan worder verkilard door a) toenemende of afnemende cardiovasculaire mortaliteit en veranderend aantal terugkerende infarcten gedurende de afgelopen decennia? b) veranderende kenmerken van studiepopulaties gedurende de afgelopen decennia? Voor deze studie gebruikten we de gegevens van de meest recent gepubliceerde meta-analyse betreffende effecten van psycho-educatic programma's op cardiovasculaire mortaliteit en morbiditeit in patienten met coronarialijden (Dusseldorp et al., 1999), waarin slechts studies werden geincludeerd van relatief hoge kwaliteit. Aanvullend werden de literatuurbestanden MEDLINE en PSYCHlit systematisch doorzocht om studies te identificeren die zijn gepubliceerd vanaf 1998. Vijfenveertig studies werden geincludeerd in de studie, die alle betrekking hadden op de effecten van psycho-educatie programmą's voor patienten met coronarialijden. In de 11 studies die het aantal terugkerende infarcten na 12 maanden rapporteerden, correleert publicaticjaar van de studie niet met het effect van de interventies op de hoeveelheid terugkerende infarcten. Acht studies rapporteren 12-maands cardiovasculaire mortaliteitscijfers, en in deze studies correleert publicatiejaar negatief met de odds ratio van cardiovasculaire mortaliteit; oudere studies rapporteren dus grotere gunstige effecten van interventies op cardiovasculaire mortaliteit dan meer recent gepubliceerde studies. Hoewel mortaliteit lijkt te dalen in deze acht studies is deze daling niet significant, en is de dalende mortaliteit niet gecorreleerd aan de kleinere effecten van de interventies op mortaliteit. De kleinere 
effecten op mortaliteit gaan echter gepaard met een toenemende gemiddelde leeftijd van de studiepopularie in recentere studies. We bestudeerden veranderingen in populatiekenmerken in 45 studies, en vonden dat patienten met coronarialijden die in recentere studies werden geincludeerd gemiddeld ouder zijn dan patienten in oudere studies. Bovendien worden recente studies gekenmerkt door een hoger percentage vrouwen in vergelijking met oudere studies. De veranderende kenmerken van de populatie zijn gecorreleerd aan veranderende inclusiecriteria in de studies. Deze resultaten laten zien dat de externe validiteit van meta-analyses afneemt. We bediscussiëren dat de veranderende patientkenmerken het draagvlak van de interventies aantast. Interventies werden ontworpen voor en gericht op de verbetering van de gezondheid, van jonge mannen, terwijl in studies steeds vaker oudere en vrouwelijke parienten worden geincludeerd.

Deskundigen benadrukken de noodzaak van zorg op maat in de cardiorevalidatie, maar vooralsnog ontbreken de studies die de verdiensten hiervan aantonen. Het belangrijkste doel van hoofdstuk 5 was om. de kwaliteit te onderzoeken van een screeningsinstrument dat was ontwikkeld om patienten vooraf te selecteren voor een multifactoriële interventie, en om te bepalen of aldus geselecteerde patienten meer baat hadden bij een mulufactoriele interventie dan patienten die niet voldeden aan de selectiecriteria. Alle 204 patiënten die werden geincludeerd in bovenbeschreven effectstudie werden gescreend met als doel patienten te selecteren met een ongunstig risicoprofiel voor coronarialijden. De hypothese dat patiènten met een slecht profiel meer baat zouden hebben van de interventie dan patienten met een gunstig profiel werd bestudeerd door een interactievariabele in de covariantic analyses op te nemen waarmee de effecten werden bestudeerd van de interventie op klinische en psychologische risicofactoren. De geselectecrde patiënten bleken inderdaad ongunstiger niveaus van risicofactoren te hebben dan niet geselecteerde patienten. De interactievariabele was ntet significant in de analyses van de acht bestudeerde risicofactoren, hetgeen impliceert dat de effecten van de interventie niet verschilden tussen patienten met of zonder relatief ongunstig risicoprofiel. Een post-hoc uitgevoerde sub-analyse met patienten met een relatief ongunstig risicoprofiel toonde aan dat de interventie voor deze groep patienten (significant) effectief was in het reduceren van het cholesterol- en bloeddrukniveau, terwiị gunstige effecten met betrekking tot quetelet-index, vitale uitputting en kwaliteit van leven een trend voor significantic bereikten. Fien subanalyse met patienten met gunstig risicoprofiel toonde aan dat de interventie in deze groep patiènten significant effectief was in het reduceren van én risicofactor, te weten rype A gedrag. Het gebruik van pre-selectic van patiènten voor multifactoriele interventies moet bestudeerd worden in grotere en liefst zickere studiepopulaties. Hoewel in de hoofdanalyses geen statistisch bewijs werd. gevonden voor de effectiviteit van pre-selectie voor multifactoriele interventies, duiden de subgroep analyses erop dat de efficiëntie van cardiorevalidatie mogelijk kan worden verhoogd door deze te beperken tot patiènten met coronarialijden die een slecht risicoprofiel hebben.

In de algemene discussie tenslotte worden de resultaten van de studie kort samengevat en worden hypothetische verklaringen voor het effect van de 
interventie (of het gebrek daaraan) en pre-selectie besproken, waarbij de belangrijkste beperkingen van de studie in ogenschouw worden genomen. Implicaties die de bevindingen hebben voor de klinische praktijk worden besproken, en er worden suggesties gedaan voor toekomstig onderzoek. Er wordt onder meer besproken dat toekomstig onderzoek meer aandacht zou moeten hebben voor het bestuderen van effectieve en niet-effectieve elementen van psychologische interventies. 


\section{DANKWOORD}

De afgelopen jaren heb ik steun mogen ontvangen van veel mensen, zonder wie ik dit project niet tot een goed einde had kunnen brengen. Ik heb vaak verlangd naar het moment waarop ik dit dankwoord zou kunnen schrijven. Nu het dan zover is blijkt het toch moeilijker dan ik had gedacht, niet in de laatste plaats doordat ik haast zou vergeten wie mij in de beginjaren (alweer enige tijd geleden) hebben geholpen en gesteund. Mocht ik in het onderstaande dan ook mensen over het hoofd hebben gezien; mijn excuses hiervoor.

Op de eerste plaats gaat mijn dank uit naar Ad Appels en Frits Bär, die de afgelopen jaren steeds voor me klaarstonden om over mijn geschreven stukken tot in het eindeloze te discussiëren. Het hebben van twee promotoren is niet makkelijk op de momenten dat hun ideeen tegenstrijdig zijn. Dit probleem was gelukkig opgelost door in de laatste jaren steeds met jullie gezamenlijk af te spreken. Ad, de eerste tijd was je slechts op de achtergrond bij het project betrokken, maar je begeleiding werd intensief toen de omstandigheden dat vroegen. Ik ben je dankbaar voor je begeleiding die ik vaak enthousiasmerend en creaticf vond en dic ook de laatste tijd na je vertrek bij de vakgroep doorging, zelfs op zaterdagen in de tuin bij Frits als ik door mijn werk verhinderd was door de week af te spreken. Frits, ondanks je drukke werkzaamheden was je altijd beschikbaar als ik je nodig had; dit waardeer ik enorm. Ik heb je steun altijd gevoeld zelfs in tijden dat ik zelf grote twijfels had of ik dit project zou kunnen of willen volbrengen. Bedankt ook voor je relevante bijdragen die ervoor zorgden dat ik de medische kant van het project in het oog bleef houden. Je was steeds bijzonder snel met je commentaar; mogen velen daar een voorbeeld aan nemen.

Alle hartpatiënten die hebben deelgenomen aan het onderzoek will ik bedanken voor hun grote inzet bij het invullen van de vele vragenlijsten en het beantwoorden van de vragen tijdens de interviews.

Mijn dank gaat ook uit naar de therapeuten en voorlichters van het revalidatieprogramma en naar hen die de eerste jaren declnamen aan de begeleidingscommissie. Hugo Corstiens, Paul Falger, André Franssen, Esther van den Hogen, Judith Jehle, Hugo Lambriex, Eline Lindeman, Peter Voorhoeve en Jos Widdershoven; bedankt voor jullie tomeloze inzet en voor de goede adviezen. Paul ook bedankt voor je nutrige bijdragen aan de artikelen. Amold Kester, bedankt voor je onmisbare ondersteuning in de wereld die statistiek heet, en Pim Dassen, bedankt voor je ondersteuning in de randomisatic. Dankzij de steun van de poli cardiologie was het steeds mogelijk patientafspraken te controleren of in te plannen. Ik realiseer me hoe hectisch het vaak is op de poli, dus voor hun hulp wil ik in het bijzonder Marie-Louise en Els bedanken. Ook de verplecgafdelingen: bedankt. 
Dank ben ik ook verschuldigd aan Willy Dubois en Jeannine van Soest, die met mij vele patiënten hebben geinterviewd. Suzanne Kaptein dank ik voor het invoeren van data, en Erik van Dijk voor het scoren van interviews. Gerrie van Wunnick, bedankt voor de ondersteuning van de video-opnames. Ook van jou heb ik meer dan alleen technische ondersteuning gekregen, zo kon ik bij je klagen als dingen niet gingen zoals ik ze graag had gewild.

Mijn dank gaat ook uit naar Adhesie, waar ik nu ruim drie jaar met veel plezier. werk. Het afronden van mijn proefschrift vormde hierbij een extra belasting. Ik ben dan ook dankbaar voor de steun die ik bij Adhesie heb gevoeld van vele collega's. In het bijzonder wil ik noemen de ondersteuning van Roelof ten Doesschate, Marc Verbraak en Peter Bournas.

Het onderzoek was een hele klus en behalve hele leuke momenten heb ik ook veel zware momenten gehad. Deze heb ik gelukkig kunnen delen met mijn intervisieclubje: Anja, Dianne, Helga, Inez en Manon. Ik wil jullie niet alleen bedanken voor de nuttige en leerzame discussies die we hadden over onze onderzocken, maar ook en bovenal voor het lotgenotencontact dat we hadden.

Natuurlijk ben ik alle vrienden en familie die interesse toonden in de voortgang van mijn proefschrift dankbaar voor hun oprechte belangstelling. In het bijzonder denk ik hierbij aan tante leentje, ome Bert, tante Joke en ome Wim, Thomas en Remeo. Ook wil ik in dit verband noemen Anke, Annelies, Anthony, Arabelle, David, Geraldine, Helga, John, Norbert, Peter, Ramon, Tonny, en Vincent. In het bijzonder wil ik Tonny en David bedanken voor de financiële steun die ik mocht ontvangen van Suprapolix: super!

Jeannine, je was niet alleen een steun bij het interviewen van patienten, je bent daarnaast een vriendin geworden door wie ik Maastricht extra ben gaan waarderen. Ik heb onze etentjes, kletsavondjes en filmhuis-avonden (laat ik het fitnessen maar overslaan) heel erg gezellig gevonden. Behalve lachen kan ik ook bij je uithuilen, bedankt!!

Maastricht is een leuke stad, en op de vakgroep had ik leuke collega's die ik het best tezamen kan bedanken voor de leuke tijd die ik op de UM heb gehad.

Nict voor niets heb ik uit mijn ex-collega's mịn twee paranimfen gekozen. Anja, je bent al deze jaren al mijn paranimf-in-spé geweest. Ik ben blij dat het er nu dan toch van komt. Ik heb dankzij jou mijn tweede (of derde?) puberteit met veel plezier meegemaakt, ik zal onze gezellige avondjes stappen in Maastricht nooit vergeten. Het waren goede tijden. Bovendien hebben we onze hoogtepunten en dips als AIO samen doorgemaakt en dit is de afgelopen jaren voor mij echt onmisbaar geweest. Bedankt!! Anock, als vriendin en ex-kamergenootje wil ik jou ook bedanken voor een hele leuke tijd in Maastricht en op de vakgroep. Soms denk ik echter wel: zouden we ons onderzoek wat sneller hebben afgerond als ik minder vaak met jou had zitten kletsen?! Maar het was gezellig! 
Het salaris van een AIO gaat niet over rozen, zeker niet in de beginjaren. Gelukkig kreeg ik regelmatig overlevingsdozen met eten en WC-papier van jou, Jam: bedankt, ook voor je leuke bezoekjes aan Maastricht. Gelukkig had ik ook meer dan slechts mijn basisbehoeften bevredigd dankzij de financiële extraatjes die ik de eerste jaren van jou kreeg, pa. Natuurlijk kan ik mijn twee 'wederhelften' niet vergeten: Corien en Irma, bedankt voor jullie gezelligheid en steun! Ik heb eigenlijk nooit gemerkt dat jullie zo ver weg woonden, ik zag jullie toch zo vaak?! Vooral het sombere gevoel dat ik kreeg als jullie na een weekendje bij mij weer naar huis gingen geeft wel aan hoe leuk ik het altijd heb gevonden.

Last but not least, bedankt Camiel, bedankt lieve schat. Ik weet dat ik er door de drukte van promoveren en opleiding en werk in Almelo de afgelopen jaren niet altijd zo vaak voor je ben (en niet zo vaak bij je ben) als jii en ik wel zouden willen. Ik besef dat dit ook voor jou niet altijd makkelijk is, toch klaag je nooit en ben je er voor me. Bedankt voor je steun!! 



\section{ABOUT THE AUTHOR}

Ellen Sebregts werd geboren op 2 maart 1969 in Wouwse Plantage. Na het behalen van het VWO diploma aan het Mollerlyceum te Bergen op Zoom, ging zij in 1987 psychologie studeren aan de Katholieke Universiteit Brabant te Tilburg. In 1993 behaalde zij het doctoraal diploma van de afstudeerrichting Toegepaste Sociale Psychologie met de aantekening 'met genoegen'. In aansluiting hierop werkte zij tot medio 1995 als assistent-onderzoeker bij het NIVEL te Utrecht. Van september 1995 tot januari 2001 werkte zij als Assistent In Opleiding (AIO) bij de vakgroep Medische Psychologie - later Departement Medische, Klinische en Experimentele Psychopathologie - van de Universiteit Maastricht. Deze aanstelling mondde uit in het schrijven van dit proefschrift.

Ellen Sebregts is sinds 2001 als behandelaar werkzaam bij Adhesie GGZ MiddenOverijssel. Adhesie biedt geintegreerde geestelijke gezondheidszorg in de regio's Almelo, Deventer en Hellendoorn. Sinds januari 2003 volgt zij hier de postdoctorale beroepsopleiding tot GZ Psycholoog.

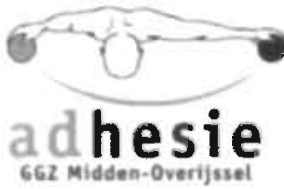


